\title{
A positivity-preserving high-order weighted compact nonlinear scheme for compressible gas-liquid flows
}

\author{
Man Long Wong ${ }^{\mathrm{a}, 1, *}$, Jordan B. Angel ${ }^{\mathrm{b}, 1}$, Michael F. Barad ${ }^{\mathrm{b}}$, Cetin C. Kiris ${ }^{\mathrm{b}}$ \\ ${ }^{a}$ Science and Technology Corporation, Moffett Field, CA 94035, United States \\ ${ }^{b}$ NASA Ames Research Center, Moffett Field, CA 94035, United States
}

\begin{abstract}
We present a robust, highly accurate, and efficient positivity- and boundedness-preserving diffuse interface method for the simulations of compressible gas-liquid two-phase flows with the five-equation model by Allaire et al. [1] using high-order finite difference weighted compact nonlinear scheme (WCNS) in the explicit form. The equation of states of gas and liquid are given by the ideal gas and stiffened gas laws respectively. Under a mild assumption on the relative magnitude between the ratios of specific heats of the gas and liquid, we can construct limiting procedures for the fifth order incremental-stencil WCNS (WCNS-IS) with the first order Harten-Lax-van Leer contact (HLLC) flux such that positive partial densities and squared speed of sound can be ensured in the solutions, together with bounded volume fractions and mass fractions. The limiting procedures are discretely conservative for all conservative equations in the five-equation model and can also be easily extended for any other conservative finite difference or finite volume scheme. Numerical tests with liquid water and air are reported to demonstrate the robustness and high accuracy of the WCNS-IS with the positivity- and boundedness-preserving limiters even under extreme conditions.
\end{abstract}

Keywords: positivity-preserving, boundedness-preserving, weighted essentially non-oscillatory (WENO), diffuse interface method, multi-phase, shock-capturing

\section{Introduction}

In the numerical computations of compressible flows, simulations cannot proceed when negative density or squared speed of sound appears because the system of equations becomes ill-posed. This problem is more pronounced for extreme applications such as those in astrophysics where strong shocks, rarefactions, or blast waves may exist in the simulations. While one can have successful simulations with some robust (or even positivity-preserving) first or second order shock-capturing schemes [11, 27, 3, 28, 15], these schemes are numerically very dissipative and are inefficient for scale-resolving simulations, such as large eddy simulations (LESs) or direct numerical simulations (DNSs).

\footnotetext{
* Corresponding author.

${ }^{1}$ These authors contributed equally to the development of positivity- and boundedness-preserving limiters.

Preprint submitted to Journal of Computational Physics

February 11, 2021
} 
Over the decades, many high-order accurate shock-capturing schemes with more localized numerical dissipation and higher resolution were developed for scale-resolving simulations $[42,22,10,4,25,19,24,12,49,44]$. While these high-order shock-capturing schemes have a certain degree of robustness for problems involving shocks and other kinds of discontinuities, there is still no guarantee of having successful simulations for severe problems using those schemes. Replacing the negative density or squared sound speed with positive ones is not conservative and may trigger other numerical issues such as spurious oscillations.

In recent years, many positivity-preserving limiters $[53,54,55,20]$ have been developed for high-order shock-capturing schemes. These limiters can preserve positivity of density and squared speed of sound for compressible flows. Motivated by the positivity-preserving technique in Perthame and Shu [33], Zhang and Shu [53] developed positivity-preserving high-order discontinuous Galerkin (DG) schemes for Euler equations. The method was later extended to Euler equations with source terms [54]. Positivity-preserving limiters specifically designed for high-order finite difference schemes didn't appear until the works by Zhang and Shu [55] and Hu et al. [20] where extreme problems could be successfully simulated with the finite difference weighted essentially non-oscillatory (WENO) schemes using positivity-preserving limiters. These methods are conservative as the limiters are applied to the fluxes directly in the conservation form. All of the positivity-preserving limiters aforementioned were designed for compressible single-phase flows. The appearance of non-physical states also happens in compressible multi-phase simulations. In general it is more likely for non-physical states to appear due to higher density gradients across material interfaces in the related applications such as supersonic combustion, cavitation erosion, break-up of high-speed liquid jets, water-based acoustic suppression systems, etc. In addition to having negative density and squared speed of sound, the solutions are also considered non-physical if mass fractions or volume fractions are not bounded between zero and one. In order to address the numerical issues, some boundedness-preserving diffuse interface methods $[39,21]$ in the Eulerian framework have been proposed. Shen et al. [39] adopted the maximum-principle-satisfying limiter by Zhang and Shu [52] for a space-time conservation element and solution element (CE/SE) scheme. The scheme can ensure the boundedness of volume fractions in the five-equation model by Allaire et al. [1] for multi-phase flows. Another thermodynamicsconsistent boundedness-preserving scheme by Jain et al. [21] for the same flow model was developed with the use of interface-regularization terms. Although both methods can preserve boundedness of volume fractions, non-physical states can still appear in the multi-phase simulations since partial densities or squared sound speed can still become negative. A positivity-preserving high-order method by Cheng and Shu [7] was proposed for multi-phase simulations in the Lagrangian framework. Compared to Eulerian diffuse interface methods, Lagrangian methods can be more accurate at material interfaces since the computational mesh moves with the fluids. On the other hand, diffuse interface methods in the Eulerian framework is more attractive for flows involving large deformations as the degree of deformations is limited by mesh distortions in Lagrangian methods [35]. Motivated by the need for the simulations of water sound suppression systems in rocket launch environments that involve interactions between strong shocks and complex airwater interfaces, we propose a high-order positivity-preserving diffuse interface method in the Eulerian framework targeting gas-liquid two-phase flows with large deformations, where the gas and liquid are described by the ideal gas and stiffened gas equation of 
states respectively. Unlike the previous works $[17,18]$ that are based on the homogeneous relaxation model, the flows in this work are described by the five-equation model by Allaire et al. [1]. Under a necessary but generally valid assumption that the ratio of specific heat of the gas is smaller than that of the liquid, the numerical method can ensure physically admissible states with positive partial density of each phase, positive squared sound speed, and bounded volume fractions and mass fractions.

The high-order shock-capturing scheme used in this work is based on the explicit finite difference formulation of weighted compact nonlinear schemes (WCNSs) [10, 31, 51, 29, 9, $30,49]$ and the nonlinear weighting technique of the incremental-stencil WENO (WENOIS) scheme [48]. The use of WCNS as a diffuse interface method for the five-equation model [1] has already been demonstrated by Wong and Lele [49] but it is only applied for single-phase flows with mixture of ideal gases. The WENO-IS scheme was originally designed as a finite volume scheme by Wang et al. [48] for compressible multi-phase flows with shocks and material interfaces using the same five-equation model. Although the robustness of the scheme was demonstrated in that paper, the finite volume approach is computationally more expensive than the finite difference WCNS for multi-dimensional problems, while the orders of accuracy are similar [36]. The WCNS with the WENO-IS nonlinear weighting technique, WCNS-IS, presented in this work is more efficient and has similar robustness in minimizing spurious oscillations in simulations.

In this work, we first show the convexity of the physically admissible set of solution states, under the mild assumption on the relative magnitude between the ratios of specific heats of the ideal gas and the liquid. We then prove the positivity-preserving and boundedness-preserving properties of the first order Harten-Lax-van Leer contact (HLLC) flux for gas-liquid flows with our choice of the advection velocity of the material interface using the convexity of the admissible set. Based on the positivity- and boundedness-preserving properties of the first order HLLC flux, we propose a limiter to blend a flux from any Cartesian conservative high-order shock-capturing schemes with the HLLC flux. The flux limiter together with a limiter for WENO interpolation can ensure the positivity-preserving and boundedness-preserving properties of the overall scheme. The fifth order accurate WCNS-IS formulation presented in this work is also proved mathematically and shown numerically to be high-order accurate in smooth advection problems, while robust because of the use of lower order interpolation near discontinuities such as shocks or material interfaces. We have demonstrated that the WCNS-IS scheme with the positivity- and boundedness-preserving limiters, PP-WCNS-IS, can successfully simulate very intense one-dimensional (1D) and two-dimensional (2D) air-water problems such as Mach 10 shock-water column interaction and Mach 100 water jet problems. The results also show that smaller errors are produced at shocks and material interfaces, and fine-scale flow features such as vortices are better captured with the high-order scheme compared to the first order HLLC scheme due to more localized numerical dissipation and higher resolution of the former method. All of the numerical tests highlight the robustness of the overall positivity- and boundedness-preserving finite difference scheme, and demonstrate the method as a highly accurate diffuse interface method for scale-resolving compressible gas-liquid simulations. 


\section{Governing equations}

The five-equation single-velocity, single-pressure model proposed by Allaire et al. [1] for compressible two-phase flows is considered in this work. The flow model has the following form:

$$
\begin{aligned}
\partial_{t}\left(\alpha_{1} \rho_{1}\right)+\nabla \cdot\left(\alpha_{1} \rho_{1} \boldsymbol{u}\right) & =0, \\
\partial_{t}\left(\alpha_{2} \rho_{2}\right)+\nabla \cdot\left(\alpha_{2} \rho_{2} \boldsymbol{u}\right) & =0, \\
\partial_{t}(\rho \boldsymbol{u})+\nabla \cdot(\rho \boldsymbol{u} \otimes \boldsymbol{u})+\nabla p & =0, \\
\partial_{t} E+\nabla \cdot[(E+p) \boldsymbol{u}] & =0, \\
\partial_{t} \alpha_{1}+\boldsymbol{u} \cdot \nabla \alpha_{1} & =0,
\end{aligned}
$$

where $\boldsymbol{u}$ is the velocity vector and $p$ is the mixture pressure. $\boldsymbol{u}=u$ and $\boldsymbol{u}=(u v)^{T}$ for $1 \mathrm{D}$ and $2 \mathrm{D}$ cases respectively (similar extension for the three-dimensional case). $\alpha_{k}$ and $\rho_{k}$ are respectively the volume fraction and phasic density of phase $k$, where $k=1,2$. $\alpha_{k} \rho_{k}=\rho Y_{k}$ is called partial density of phase $k$, where $\rho=\alpha_{1} \rho_{1}+\alpha_{2} \rho_{2}$ is the mixture density and $Y_{k}$ is mass fraction of phase $k . E=\rho\left(e+|\boldsymbol{u}|^{2} / 2\right)$ is the mixture total energy per unit volume, where $e$ is the mixture specific internal energy. Also, $\alpha_{1}+\alpha_{2}=1$ and $\rho e=\alpha_{1} \rho_{1} e_{1}+\alpha_{2} \rho_{2} e_{2}$, where $e_{k}$ is the phasic specific internal energy of phase $k$. The system is closed by the mechanical equilibrium and equation of state of each phase ${ }^{2}$. The stiffened gas equation of state is chosen in this work because of its popularity for gases and liquids. The equation of state was first proposed by Harlow and Amsden [16]. The stiffened gas equation of state of each phase is given by:

$$
\frac{p_{k}}{\gamma_{k}-1}+\frac{\gamma_{k} p_{k}^{\infty}}{\gamma_{k}-1}=\rho_{k} e_{k}
$$

where $\gamma_{k}$ and $p_{k}^{\infty}$ are fitting parameters for each of the fluids. $\gamma_{k}$ is the ratio of specific heats that is greater than one and $p_{k}^{\infty}$ is non-negative. The stiffened gas equation of state is reduced to the ideal gas equation of state if $p_{k}^{\infty}=0$. With the mechanical equilibrium assumption, i.e. $p_{1}=p_{2}=p$, we can obtain the mixture equation of state by multiplying equation (6) by $\alpha_{k}$ for each phase and then summing over all the phases:

$$
\frac{p}{\bar{\gamma}-1}+\frac{\bar{\gamma} \overline{p^{\infty}}}{\bar{\gamma}-1}=\rho e
$$

where $\bar{\gamma}$ and $\overline{p^{\infty}}$ are properties of the mixture. They can be defined by the following relations:

$$
\begin{aligned}
& \frac{1}{\bar{\gamma}-1}=\frac{\alpha_{1}}{\gamma_{1}-1}+\frac{\alpha_{2}}{\gamma_{2}-1} \\
& \frac{\bar{\gamma} \overline{p^{\infty}}}{\bar{\gamma}-1}=\frac{\alpha_{1} \gamma_{1} p_{1}^{\infty}}{\gamma_{1}-1}+\frac{\alpha_{2} \gamma_{2} p_{2}^{\infty}}{\gamma_{2}-1}
\end{aligned}
$$

\footnotetext{
${ }^{2}$ In this work, each phase consists of one species.
} 
We define the conservative variable vector as $\mathbf{W}=\left(\rho_{1} \alpha_{1} \rho_{2} \alpha_{2} \rho \boldsymbol{u} E \alpha_{1}\right)^{T 3}$. In this work, we also define the set of admissible states as,

$$
G=\left\{\mathbf{W}=\left(\begin{array}{c}
\alpha_{1} \rho_{1} \\
\alpha_{2} \rho_{2} \\
\rho \boldsymbol{u} \\
E \\
\alpha_{1}
\end{array}\right) \mid 0 \leq \alpha_{1} \leq 1, \alpha_{1} \rho_{1} \geq 0, \alpha_{2} \rho_{2} \geq 0, \rho c^{2}>0\right\} .
$$

This requires boundedness of the volume fractions, positivity of the partial densities, and positivity of the squared speed of sound $c^{2}$. The positive squared speed of sound implies that the system of equations remains hyperbolic with real wave speeds. Note that the positive partial densities also mean that all mass fractions $Y_{k}$ are bounded between zero and one. The flow model has a mixture speed of sound $c$ given by [1]:

$$
\begin{aligned}
\rho c^{2} & =\bar{\gamma}\left(p+\overline{p^{\infty}}\right) \\
& =\bar{\gamma}(\bar{\gamma}-1)\left(\rho e-\overline{p^{\infty}}\right)=\bar{\gamma}(\bar{\gamma}-1)\left(E-\frac{1}{2} \frac{|\rho \boldsymbol{u}|^{2}}{\rho}-\overline{p^{\infty}}\right) .
\end{aligned}
$$

Both $\bar{\gamma}$ and $\overline{p^{\infty}}$ only depend on $\alpha_{1}$ in $\mathbf{W}$.

It is obvious that $\alpha_{k}$ and $\alpha_{k} \rho_{k}$ are both concave functions of the conserved variables W. Since all $\gamma_{k}$ are greater than one, $\bar{\gamma}>1$ if $0 \leq \alpha_{1} \leq 1$. Therefore, an equivalent physically admissible set is,

$$
G=\left\{\mathbf{W}=\left(\begin{array}{c}
\alpha_{1} \rho_{1} \\
\alpha_{2} \rho_{2} \\
\rho \boldsymbol{u} \\
E \\
\alpha_{1}
\end{array}\right) \mid 0 \leq \alpha_{1} \leq 1, \alpha_{1} \rho_{1} \geq 0, \alpha_{2} \rho_{2} \geq 0, \rho e-\bar{p}^{\infty}>0\right\} .
$$

Lemma 1. If $p_{2}^{\infty}=0$ ( $\left.p_{1}^{\infty}=0\right), \gamma_{1} \geq \gamma_{2}\left(\gamma_{2} \geq \gamma_{1}\right)$ and $0 \leq \alpha_{1} \leq 1$, the function $\rho e-\bar{p}^{\infty}$ is a concave function of the conserved variables $\mathbf{W}$.

Proof. The non-zero eigenvalues of the Hessian matrix of the function are:

$$
\left\{-\frac{1}{\alpha_{1} \rho_{1}+\alpha_{2} \rho_{2}},-\frac{2|\rho \boldsymbol{u}|^{2}+\left(\alpha_{1} \rho_{1}+\alpha_{2} \rho_{2}\right)^{2}}{\left(\alpha_{1} \rho_{1}+\alpha_{2} \rho_{2}\right)^{3}},-\frac{\partial^{2} \bar{p}^{\infty}}{\partial \alpha_{1}^{2}}\right\} .
$$

The first non-zero eigenvalue does not exist for the 1D case. If the last non-zero eigenvalue is non-positive, then the lemma is proved since all eigenvalues are non-positive. Here, we consider the $p_{2}^{\infty}=0$ case and the other case is symmetric. For $p_{2}^{\infty}=0$ we have,

$$
\frac{\partial^{2} \bar{p}^{\infty}}{\partial \alpha_{1}^{2}}=2 \frac{\gamma_{1} p_{1}^{\infty}}{\gamma_{1}-1} \frac{\frac{1}{\gamma_{1}-1}-\frac{1}{\gamma_{2}-1}}{\left(\frac{\alpha_{1}}{\gamma_{1}-1}+\frac{\alpha_{2}}{\gamma_{2}-1}+1\right)^{2}}\left[\frac{\alpha_{1}\left(\frac{1}{\gamma_{1}-1}-\frac{1}{\gamma_{2}-1}\right)}{\left(\frac{\alpha_{1}}{\gamma_{1}-1}+\frac{\alpha_{2}}{\gamma_{2}-1}+1\right)}-1\right],
$$

and it is clear that if $\gamma_{1} \geq \gamma_{2}$, the last eigenvalue is less than or equal to zero. Note that if $\gamma_{2}>\gamma_{1}$, the function cannot be concave. In this work, we assume that one of the phases is an ideal gas with $p_{k}^{\infty}=0$.

\footnotetext{
${ }^{3}$ Strictly speaking, $\alpha_{1}$ is not a conservative variable.
} 
Theorem 1. The set $G$ is a convex set.

Proof. This is an immediate consequence of Lemma 1 and Jensen's inequality.

The proof of convexity of $G$ relies on the assumption that the ratio of specific heats of the liquid $\left(p_{k}^{\infty}>0\right)$ is larger than that of the ideal gas $\left(p_{k}^{\infty}=0\right)$. However, this is a very mild assumption based on most gas-liquid test problems found in previous literature. The ideal gases considered in the previous works are usually either monatomic gases or air, where the ratios of specific heats are around 1.67 and 1.4 respectively. When the stiffened gas equation of state is chosen for the liquid in tests, the most popular liquid used is water, where the ratio of specific heats mostly ranges between $4-7$ [34, 43, 8, 48, 32]. A rare but justified choice of $\gamma=1.932$ is utilized in [5] but that ratio of specific heats is still larger than those of monatomic gases or air. Other liquids commonly found in the literature include ethanol [32] with $\gamma=2.1$ and mercury [45] with $\gamma=8.2$. Both liquids also have larger specific heat ratios than the ideal gases considered.

In the following sections, we make the assumption that ratio of specific heats of liquid is larger than that of ideal gas and formulate our numerical method by taking advantage of the convexity of $G$ such that the solution update is a convex combination of states already in $G$, thus is also in $G$.

\section{First order positivity- and boundedness-preserving scheme with HLLC Riemann solver}

The flux given by the HLLC Riemann solver is illustrated in this section. For simplicity, a $2 \mathrm{D}$ case with domain $\left[x_{a}, x_{b}\right] \times\left[y_{a}, y_{b}\right]$ is considered with the following equation in compact form:

$$
\partial_{t} \mathbf{W}+\partial_{x} \mathbf{F}^{x}(\mathbf{W})+\partial_{y} \mathbf{F}^{y}(\mathbf{W})+\boldsymbol{\Sigma}(\mathbf{W}, \nabla \mathbf{W})=\mathbf{0},
$$

where

$$
\begin{aligned}
\mathbf{W} & =\left(\begin{array}{c}
\alpha_{1} \rho_{1} \\
\alpha_{2} \rho_{2} \\
\rho u \\
\rho v \\
E \\
\alpha_{1}
\end{array}\right), \quad \mathbf{F}^{x}(\mathbf{W})=\left(\begin{array}{c}
\alpha_{1} \rho_{1} u \\
\alpha_{2} \rho_{2} u \\
\rho u^{2}+p \\
\rho v u \\
(E+p) u \\
0
\end{array}\right), \quad \mathbf{F}^{y}(\mathbf{W})=\left(\begin{array}{c}
\alpha_{1} \rho_{1} v \\
\alpha_{2} \rho_{2} v \\
\rho u v \\
\rho v^{2}+p \\
(E+p) v \\
0
\end{array}\right), \\
\boldsymbol{\Sigma}(\mathbf{W}, \nabla \mathbf{W}) & =\left(\begin{array}{c}
0 \\
0 \\
0 \\
0 \\
u \\
u
\end{array}\right) .
\end{aligned}
$$

If the domain is discretized uniformly into a Cartesian grid with $N_{x} \times N_{y}$ grid points, we have the domain covered by cells $I_{i, j}=\left[x_{i-1 / 2}, x_{i+1 / 2}\right] \times\left[y_{j-1 / 2}, y_{j+1 / 2}\right]$ for $1 \leq$ $i \leq N_{x}, 1 \leq j \leq N_{y}$, where the grid midpoints are given by:

$$
x_{i+\frac{1}{2}}=x_{a}+i \Delta x, y_{j+\frac{1}{2}}=y_{a}+j \Delta y
$$


and

$$
\Delta x=\frac{x_{b}-x_{a}}{N_{x}}, \quad \Delta y=\frac{y_{b}-y_{a}}{N_{y}} .
$$

To obtain the numerical scheme with an exact or approximate Riemann solver, the numerical discretizations in different directions are treated independently. The flux in $y$ direction has similar formulation. Therefore, only the numerical discretization in $x$ direction is discussed in details in this section.

\subsection{Approximate solutions}

The discretization in $x$ direction is conducted by considering a generalized Riemann problem with a planar discontinuity initially at each grid midpoint in the $x$ direction, as shown in figure 1 . The generalized Riemann problem is reduced to a quasi-1D problem due to assumed homogeneity in other directions. The reduced quasi-1D generalized Riemann problem at midpoint $x=x_{i+1 / 2}$ between grid cells located at $\left(x_{i}, y_{j}\right)$ and $\left(x_{i+1}, y_{j}\right)$ is hence formulated as:

$$
\left\{\begin{array}{l}
\partial_{t} \mathbf{W}+\partial_{x} \mathbf{F}(\mathbf{W})+\mathbf{\Sigma}\left(\mathbf{W}, \partial_{x} \mathbf{W}\right)=0, \\
\mathbf{W}(x, t=0)= \begin{cases}\mathbf{W}_{L}, & x_{i+\frac{1}{2}}<0 \\
\mathbf{W}_{R}, & x_{i+\frac{1}{2}} \geq 0\end{cases}
\end{array}\right.
$$

where superscript " $x$ " in $\mathbf{F}^{x}$ and index " $j$ " are dropped for convenience. For first order accurate spatial approximation, $\mathbf{W}_{L}=\mathbf{W}_{i}$ and $\mathbf{W}_{R}=\mathbf{W}_{i+1}$. Higher order spatial approximation can be obtained from high-order interpolation to construct a high-order scheme which will be discussed in another section. The first order solution of Riemann problem given by equation (19) is self-similar: $\mathbf{W}(x, t)=R\left(x / t, \mathbf{W}_{i}, \mathbf{W}_{i+1}\right)$.

The exact solution to the generalized Riemann problem is computationally expensive and challenging to obtain. Approximate Riemann solvers can be used to provide approximate solutions in a less expensive way in Godunov-type schemes. Assuming the approximate waves generated at the two midpoints $x_{i \pm 1 / 2}$ do not interact under suitable Courant-Friedrichs-Lewy (CFL) condition as shown in figure 2, the approximate numerical solution at grid cell $i$ is the cell-averaged value:

$$
\begin{aligned}
\mathbf{W}_{i}^{n+1}= & \frac{1}{\Delta x} \int_{x_{i-\frac{1}{2}}}^{x_{i}} R\left(\frac{x-x_{i-\frac{1}{2}}}{\Delta t}, \mathbf{W}_{i-1}^{n}, \mathbf{W}_{i}^{n}\right) d x \\
& +\frac{1}{\Delta x} \int_{x_{i}}^{x_{i+\frac{1}{2}}} R\left(\frac{x-x_{i+\frac{1}{2}}}{\Delta t}, \mathbf{W}_{i}^{n}, \mathbf{W}_{i+1}^{n}\right) d x .
\end{aligned}
$$

Specifically since the last equation is an advection equation, the approximate solution of the volume fraction is given by:

$$
\begin{aligned}
\alpha_{1 i}^{n+1}= & {\left[\frac{1}{2} \alpha_{1 i}^{n}-\frac{u_{+, i-\frac{1}{2}} \Delta t}{\Delta x}\left(\alpha_{1 i}^{n}-\alpha_{1 i-1}^{n}\right)\right] } \\
& +\left[\frac{1}{2} \alpha_{1 i}^{n}-\frac{u_{-, i+\frac{1}{2}} \Delta t}{\Delta x}\left(\alpha_{1 i+1}^{n}-\alpha_{1 i}^{n}\right)\right] \\
\alpha_{1 i}^{n+1}= & \alpha_{1 i}^{n}-\frac{u_{+, i-\frac{1}{2}} \Delta t}{\Delta x}\left(\alpha_{1 i}^{n}-\alpha_{1 i-1}^{n}\right)-\frac{u_{-, i+\frac{1}{2}} \Delta t}{\Delta x}\left(\alpha_{1 i+1}^{n}-\alpha_{1 i}^{n}\right),
\end{aligned}
$$


where $u_{+, i-1 / 2}=\max \left\{0, u_{*, i-1 / 2}\right\}$ and $u_{-, i+1 / 2}=\min \left\{0, u_{*, i+1 / 2}\right\} . \quad u_{*, i \pm 1 / 2}$ are the approximate material wave speeds at edges $x_{i \pm 1 / 2}$ for the advection equation.

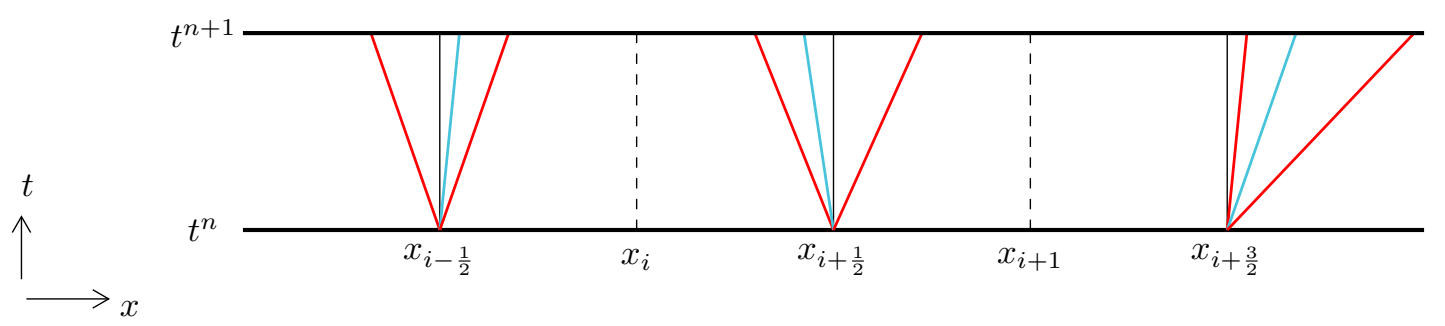

Figure 1: Illustration of a scheme with an approximate Riemann solver. Waves are generated from discontinuities initially located at grid midpoints.

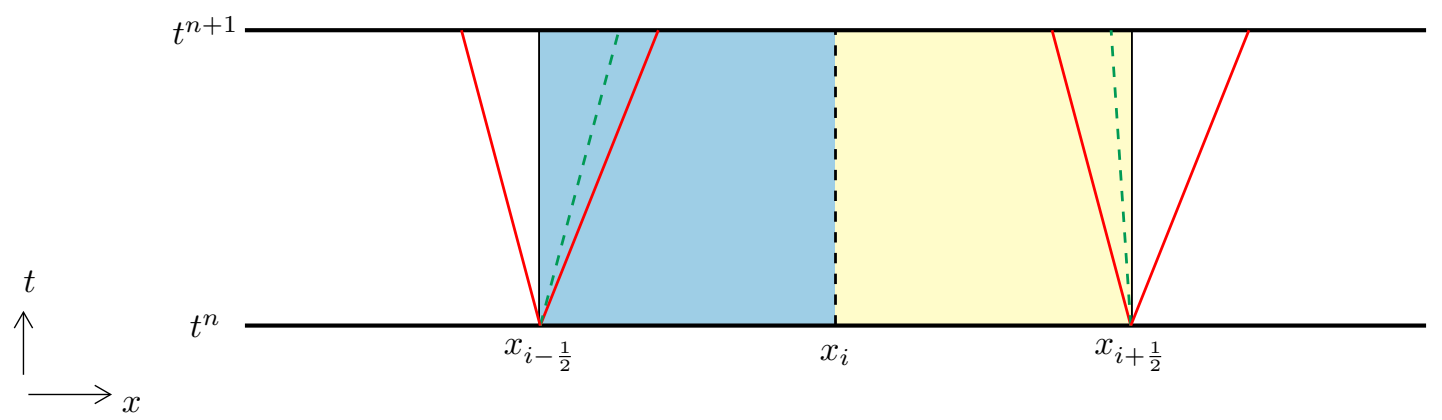

Figure 2: Approximate waves generated at the left and right edges of a cell $I_{i}=\left[x_{i-1 / 2}, x_{i+1 / 2}\right]$. The red solid lines and green dotted lines represent the locations of the approximate acoustic waves and contact waves respectively.

The HLLC discretization in a particular direction for a multi-dimensional problem can be approximated by the solutions of a quasi-1D generalized Riemann problem in that direction. The approximate solutions, $\mathbf{W}^{\text {HLLC }}$, of a quasi-1D generalized Riemann problem in $x$ direction with an initial planar discontinuity, is illustrated in figure 3 . The approximate solution to the generalized Riemann problem contains three discontinuities: one contact wave and two acoustic waves. The speed of the contact wave is denoted by $s_{*}$ while the smallest and largest acoustic wave speeds are represented by $s_{L}$ and $s_{R}$ respectively. The material wave speed for the advection equation is chosen as the contact wave speed $u_{*}=s_{*}$.

The HLLC approximate solutions in the four different regions separated by the three discontinuities are given by:

$$
\mathbf{W}^{\mathrm{HLLC}}= \begin{cases}\mathbf{W}_{L}, & \text { if } s_{L}>0, \\ \mathbf{W}_{*, L}, & \text { if } s_{L} \leq 0<s_{*}, \\ \mathbf{W}_{*, R}, & \text { if } s_{*} \leq 0 \leq s_{R}, \\ \mathbf{W}_{R}, & \text { if } s_{R}<0,\end{cases}
$$


where $L$ and $R$ are the left and right states respectively at a midpoint. With $K=L$ or $R$, the star state for the five-equation model for a $2 \mathrm{D}$ problem is given by:

$$
\mathbf{W}_{*, K}=\left(\begin{array}{c}
\chi_{*, K}\left(\alpha_{1} \rho_{1}\right)_{K} \\
\chi_{*, K}\left(\alpha_{2} \rho_{2}\right)_{K} \\
\chi_{*, K} \rho_{K} s_{*} \\
\chi_{*, K} \rho_{K} v_{K} \\
\chi_{*, K}\left[E_{K}+\left(s_{*}-u_{K}\right)\left(\rho_{K} s_{*}+\frac{p_{K}}{s_{K}-u_{K}}\right)\right] \\
\alpha_{1, K}
\end{array}\right)
$$

$\chi_{* K}$ is defined as:

$$
\chi_{* K}=\frac{s_{K}-u_{K}}{s_{K}-s_{*}} .
$$

We use the wave speeds suggested by Einfeldt et al. [11]:

$$
s_{L}=\min \left(\bar{u}-\bar{c}, u_{L}-c_{L}\right), \quad s_{R}=\max \left(\bar{u}+\bar{c}, u_{R}+c_{R}\right),
$$

where $\bar{u}$ and $\bar{c}$ are the arithmetic averages from the left and right states. For instance, $\bar{c}$ is the average of $c_{L}$ and $c_{R}$. Following Batten et al. [3], the wave speed in the star region is given by:

$$
s_{*}=\frac{p_{R}-p_{L}+\rho_{L} u_{L}\left(s_{L}-u_{L}\right)-\rho_{R} u_{R}\left(s_{R}-u_{R}\right)}{\rho_{L}\left(s_{L}-u_{L}\right)-\rho_{R}\left(s_{R}-u_{R}\right)} .
$$

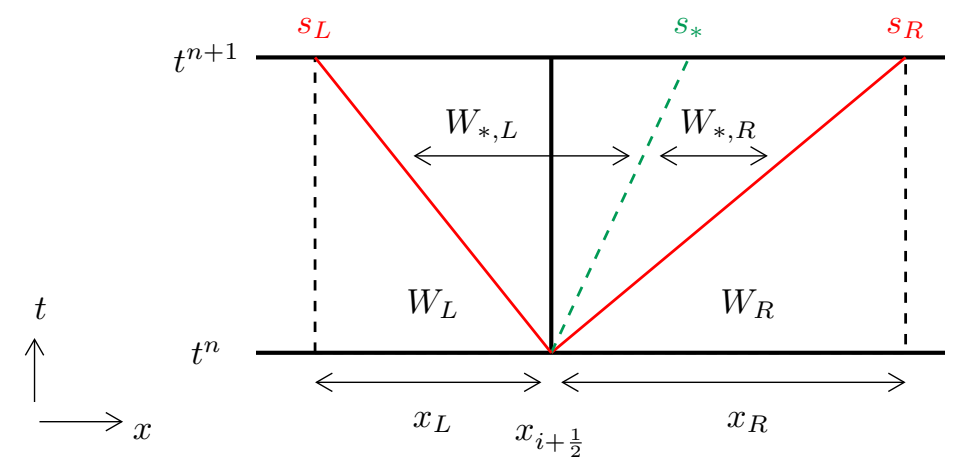

Figure 3: HLLC wave diagram at interface $x_{i+1 / 2}$. The red solid lines represent the locations of the approximate acoustic waves and the green dotted line represent the location of the contact wave.

\subsection{Approximate fluxes}

We now introduce a flux-source form that is convenient for the derivation of the fluxbased numerical discretization for the non-conservative system of equations and also the extension for high-order methods. The equation given by (15) can be rewritten as:

$$
\partial_{t} \mathbf{W}+\partial_{x} \mathbf{G}^{x}(\mathbf{W})+\partial_{y} \mathbf{G}^{y}(\mathbf{W})=\mathbf{S}(\mathbf{W}, \nabla \mathbf{W}),
$$


where

$$
\mathbf{G}^{x}(\mathbf{W})=\left(\begin{array}{c}
\alpha_{1} \rho_{1} u \\
\alpha_{2} \rho_{2} u \\
\rho u^{2}+p \\
\rho v u \\
(E+p) u \\
\alpha_{1} u
\end{array}\right), \quad \mathbf{G}^{y}(\mathbf{W})=\left(\begin{array}{c}
\alpha_{1} \rho_{1} v \\
\alpha_{2} \rho_{2} v \\
\rho u v \\
\rho v^{2}+p \\
(E+p) v \\
\alpha_{1} v
\end{array}\right), \quad \mathbf{S}(\mathbf{W}, \nabla \mathbf{W})=\left(\begin{array}{c}
0 \\
0 \\
0 \\
0 \\
0 \\
\alpha_{1} \nabla \cdot \boldsymbol{u}
\end{array}\right)
$$

The relation between fluxes $\mathbf{F}^{x}$ and $\mathbf{G}^{x}$ and that between fluxes $\mathbf{F}^{y}$ and $\mathbf{G}^{y}$ are given by:

$$
\begin{aligned}
& \mathbf{G}^{x}=\mathbf{F}^{x}+\left(\begin{array}{llllll}
0 & 0 & 0 & 0 & 0 & f_{\alpha}^{x}
\end{array}\right)^{T}, \\
& \mathbf{G}^{y}=\mathbf{F}^{y}+\left(\begin{array}{llllll}
0 & 0 & 0 & 0 & 0 & f_{\alpha}^{y}
\end{array}\right)^{T},
\end{aligned}
$$

where $f_{\alpha}^{x}=\alpha_{1} u$ and $f_{\alpha}^{y}=\alpha_{1} v$.

The fully discretized form of equation (28) with first order accurate forward Euler time integration is given by:

$$
\frac{\mathbf{W}_{i, j}^{n+1}-\mathbf{W}_{i, j}^{n}}{\Delta t}+\frac{\hat{\mathbf{G}}_{i+\frac{1}{2}, j}^{x}-\hat{\mathbf{G}}_{i-\frac{1}{2}, j}^{x}}{\Delta x}+\frac{\hat{\mathbf{G}}_{i, j+\frac{1}{2}}^{y}-\hat{\mathbf{G}}_{i, j-\frac{1}{2}}^{y}}{\Delta y}=\hat{\mathbf{S}}_{i, j},
$$

where

$$
\hat{\mathbf{S}}_{i, j}=\left(\begin{array}{c}
0 \\
0 \\
0 \\
0 \\
0 \\
\alpha_{1, i, j}^{n}\left(\frac{\hat{u}_{i+\frac{1}{2}, j}-\hat{u}_{i-\frac{1}{2}, j}}{\Delta x}+\frac{\hat{v}_{i, j+\frac{1}{2}}-\hat{v}_{i, j-\frac{1}{2}}}{\Delta y}\right)
\end{array}\right) .
$$

Only numerical approximation in $x$ direction is discussed in the following part as discretization in $y$ direction is similar. The first order accurate approximation of $\hat{\mathbf{G}}_{i \pm \frac{1}{2}, j}^{x}$ with the HLLC solutions is given by:

$$
\begin{aligned}
& \hat{\mathbf{G}}_{i-\frac{1}{2}, j}^{x}=\mathbf{G}_{i-\frac{1}{2}, j}^{x, \mathrm{HLC}}\left(\mathbf{W}_{i-1, j}, \mathbf{W}_{i, j}\right) \\
& =\mathbf{F}_{i-\frac{1}{2}, j}^{x, \mathrm{HLLC}}\left(\mathbf{W}_{i-1, j}, \mathbf{W}_{i, j}\right)+\left(\begin{array}{llllll}
0 & 0 & 0 & 0 & 0 & f_{\alpha, i-\frac{1}{2}, j}^{x, \mathrm{HLLC}} \\
\left(\mathbf{W}_{i-1, j}, \mathbf{W}_{i, j}\right.
\end{array}\right)^{T}, \\
& \hat{\mathbf{G}}_{i+\frac{1}{2}, j}^{x}=\mathbf{G}_{i+\frac{1}{2}, j}^{x, \mathrm{HLC}}\left(\mathbf{W}_{i, j}, \mathbf{W}_{i+1, j}\right) \\
& =\mathbf{F}_{i+\frac{1}{2}, j}^{x, \mathrm{HLLC}}\left(\mathbf{W}_{i, j}, \mathbf{W}_{i+1, j}\right)+\left(\begin{array}{llllll}
0 & 0 & 0 & 0 & 0 & f_{\alpha, i+\frac{1}{2}, j}^{x, \mathrm{HLLC}} \\
\left(\mathbf{W}_{i, j}, \mathbf{W}_{i+1, j}\right.
\end{array}\right)^{T},
\end{aligned}
$$

where

$$
\begin{aligned}
& \mathbf{F}_{i-\frac{1}{2}, j}^{x, \operatorname{HLLC}}\left(\mathbf{W}_{i-1, j}, \mathbf{W}_{i, j}\right)=\mathbf{F}^{x, \operatorname{HLLC}}\left(R^{\operatorname{HLLC}}\left(0, \mathbf{W}_{i-1, j}, \mathbf{W}_{i, j}\right)\right), \\
& \mathbf{F}_{i+\frac{1}{2}, j}^{x, \operatorname{HLLC}}\left(\mathbf{W}_{i, j}, \mathbf{W}_{i+1, j}\right)=\mathbf{F}^{x, \operatorname{HLLC}}\left(R^{\operatorname{HLLC}}\left(0, \mathbf{W}_{i, j}, \mathbf{W}_{i+1, j}\right)\right) .
\end{aligned}
$$


The conservative HLLC fluxes $\mathbf{F}_{i \pm \frac{1}{2}, j}^{x, \text { HLLC }}$ can be obtained with the divergence theorem [3]:

$$
\begin{aligned}
& \mathbf{F}^{x, \operatorname{HLLC}}\left(R^{\mathrm{HLLC}}\left(0, \mathbf{W}_{L}, \mathbf{W}_{R}\right)\right)= \\
& \quad \frac{1+\operatorname{sign}\left(s_{*}\right)}{2}\left(\mathbf{F}_{L}+s_{-}\left(\mathbf{W}_{*, L}-\mathbf{W}_{L}\right)\right)+\frac{1-\operatorname{sign}\left(s_{*}\right)}{2}\left(\mathbf{F}_{R}+s_{+}\left(\mathbf{W}_{*, R}-\mathbf{W}_{R}\right)\right),
\end{aligned}
$$

where

$$
s_{-}=\min \left(0, s_{L}\right), \quad s_{+}=\max \left(0, s_{R}\right) .
$$

Note that the last component of $\mathbf{F}^{x, H L L C}$ for the advection equation is zero. The discretization of the advection equation is contributed by $\hat{u}_{i \pm \frac{1}{2}, j}$ and $f_{\alpha, i \pm \frac{1}{2}, j}^{x, \mathrm{HLLC}}$ which are given by the first order accurate approximations as:

$$
\begin{aligned}
& \hat{u}_{i-\frac{1}{2}, j}=u_{*, i-\frac{1}{2}, j}=u_{*}^{\mathrm{HLLC}}\left(\mathbf{W}_{i-1, j}, \mathbf{W}_{i, j}\right)=s_{*}\left(\mathbf{W}_{i-1, j}, \mathbf{W}_{i, j}\right), \\
& \hat{u}_{i+\frac{1}{2}, j}=u_{*, i+\frac{1}{2}, j}=u_{*}^{\mathrm{HLLC}}\left(\mathbf{W}_{i, j}, \mathbf{W}_{i+1, j}\right)=s_{*}\left(\mathbf{W}_{i, j}, \mathbf{W}_{i+1, j}\right),
\end{aligned}
$$

and

$$
\begin{aligned}
f_{\alpha, i-\frac{1}{2}, j}^{x, \mathrm{HLLC}}\left(\mathbf{W}_{i-1, j}, \mathbf{W}_{i, j}\right)= & \frac{1+\operatorname{sign}\left(s_{*, i-\frac{1}{2}, j}\right)}{2}\left(\alpha_{1, i-1, j} s_{*, i-\frac{1}{2}, j}\right) \\
& +\frac{1-\operatorname{sign}\left(s_{*, i-\frac{1}{2}, j}\right)}{2}\left(\alpha_{1, i, j} s_{*, i-\frac{1}{2}, j}\right), \\
f_{\alpha, i+\frac{1}{2}, j}^{x, \mathrm{HLC}}\left(\mathbf{W}_{i, j}, \mathbf{W}_{i+1, j}\right)= & \frac{1+\operatorname{sign}\left(s_{*, i+\frac{1}{2}, j}\right)}{2}\left(\alpha_{1, i, j} s_{*, i+\frac{1}{2}, j}\right) \\
& +\frac{1-\operatorname{sign}\left(s_{*, i+\frac{1}{2}, j}\right)}{2}\left(\alpha_{1, i+1, j} s_{*, i+\frac{1}{2}, j}\right) .
\end{aligned}
$$

The expressions given above form the first order accurate solution of volume fraction given by equation (22).

Finally, the non-conservative flux $\hat{\mathbf{G}}_{i \mp \frac{1}{2}, j}^{x, \pm}$ (similarly for $\hat{\mathbf{G}}_{i, j \mp \frac{1}{2}}^{y, \pm}$ ) is introduced:

$$
\hat{\mathbf{G}}_{i \mp \frac{1}{2}, j}^{x, \pm}=\hat{\mathbf{G}}_{i \mp \frac{1}{2}, j}^{x}-\alpha_{1, i, j}\left(\begin{array}{lllllll}
0 & 0 & 0 & 0 & 0 & \left.\hat{u}_{i \mp \frac{1}{2}, j}\right)^{T},
\end{array}\right.
$$

where equation (32) can be simplified to:

$$
\frac{\mathbf{W}_{i, j}^{n+1}-\mathbf{W}_{i, j}^{n}}{\Delta t}+\frac{\hat{\mathbf{G}}_{i+\frac{1}{2}, j}^{x,-}-\hat{\mathbf{G}}_{i-\frac{1}{2}, j}^{x,+}}{\Delta x}+\frac{\hat{\mathbf{G}}_{i, j+\frac{1}{2}}^{y,-}-\hat{\mathbf{G}}_{i, j-\frac{1}{2}}^{y,+}}{\Delta y}=\mathbf{0} .
$$

Note that the components of $\hat{\mathbf{G}}_{i \mp \frac{1}{2}, j}^{x, \pm}$ (or $\hat{\mathbf{G}}_{i \mp \frac{1}{2}, j}^{x, \mathrm{HLLC}, \pm}$ more precisely) for all conservative equations, except the last advection equation, are conservative numerical fluxes and are equivalent to the corresponding components of $\mathbf{F}_{i \mp \frac{1}{2}, j}^{x, \mathrm{HLC}}$.

3.3. Proof of positivity- and bounded-preserving preservation of first order HLLC solver

For a quasi-1D problem, it is shown in equation (20) that the solution update is the convex averaging of the exact or approximate solutions to the generalized Riemann 
problem. Therefore, the HLLC Riemann solver gives physically admissible solution if all states generated are physically admissible using Jensen's inequality for integral equations. Here, the left star state is considered and the right star state can be proved to be physically admissible by symmetry.

With the definition of $s_{*}$ given by equation (27), it can be shown that $s_{L}<s_{*}[3]$. Also, since $s_{L}=\min \left(\bar{u}-\bar{c}, u_{L}-c_{L}\right), s_{L}<u_{L}$. As a result, the partial densities in the star state are positive:

$$
\begin{aligned}
\left(\alpha_{1} \rho_{1}\right)_{*, L} & =\frac{s_{L}-u_{L}}{s_{L}-s_{*}}\left(\alpha_{1} \rho_{1}\right)_{L} \geq 0 \\
\left(\alpha_{2} \rho_{2}\right)_{*, L} & =\frac{s_{L}-u_{L}}{s_{L}-s_{*}}\left(\alpha_{2} \rho_{2}\right)_{L} \geq 0 .
\end{aligned}
$$

Since $\rho_{*, L}=\left(\alpha_{1} \rho_{1}\right)_{*, L}+\left(\alpha_{2} \rho_{2}\right)_{*, L}$,

$$
\rho_{*, L}=\frac{s_{L}-u_{L}}{s_{L}-s_{*}} \rho_{L} \geq 0 .
$$

The positivity of mixture and partial densities implies all mass fractions are bounded between zero and one. As for the volume fraction, since $\alpha_{1, *, L}=\alpha_{1, L}$,

$$
0 \leq \alpha_{1, *, L} \leq 1
$$

The only remaining requirement is $(\rho e)_{*, L}>\bar{p}_{L}^{\infty}$ for positive squared speed of sound. From the definition of $(\rho e)_{*, L}$ :

$$
\begin{aligned}
(\rho e)_{*, L}= & E_{*, L}-\frac{1}{2} \frac{\left|\rho \boldsymbol{u}_{*, L}\right|^{2}}{\rho_{*, L}}=E_{*, L}-\frac{1}{2} \frac{(\rho u)_{*, L}^{2}+(\rho v)_{*, L}^{2}}{\rho_{*, L}} \\
(\rho e)_{*, L}= & \frac{s_{L}-u_{L}}{s_{L}-s_{*}} E_{L}+\frac{s_{L}-u_{L}}{s_{L}-s_{*}}\left(s_{*}-u_{L}\right)\left(\rho_{L} s_{*}+\frac{p_{L}}{s_{L}-u_{L}}\right) \\
& -\frac{1}{2} \frac{s_{L}-u_{L}}{s_{L}-s_{*}} \rho_{L}\left(s_{*}^{2}+v_{L}^{2}\right) .
\end{aligned}
$$

Therefore, we require the following inequality:

$$
\begin{aligned}
& \frac{s_{L}-u_{L}}{s_{L}-s_{*}} E_{L}+\frac{s_{L}-u_{L}}{s_{L}-s_{*}}\left(s_{*}-u_{L}\right)\left(\rho_{L} s_{*}+\frac{p_{L}}{s_{L}-u_{L}}\right)-\frac{1}{2} \frac{s_{L}-u_{L}}{s_{L}-s_{*}} \rho_{L}\left(s_{*}^{2}+v_{L}^{2}\right) \\
& \quad>\bar{p}_{L}^{\infty} \\
& E_{L}+\left(s_{*}-u_{L}\right)\left(\rho_{L} s_{*}+\frac{p_{L}}{s_{L}-u_{L}}\right)-\frac{1}{2} \rho_{L}\left(s_{*}^{2}+v_{L}^{2}\right) \\
& \quad>\bar{p}_{L}^{\infty} \frac{\left(s_{L}-u_{L}-s_{*}+u_{L}\right)}{\left(s_{L}-u_{L}\right)} \\
& (\rho e)_{L}+\frac{1}{2} \rho_{L}\left(u_{L}^{2}+v_{L}^{2}\right)+\left(s_{*}-u_{L}\right)\left(\rho_{L} s_{*}+\frac{p_{L}}{s_{L}-u_{L}}\right)-\frac{1}{2} \rho_{L}\left(s_{*}^{2}+v_{L}^{2}\right) \\
& \quad>\bar{p}_{L}^{\infty} \frac{\left(s_{L}-u_{L}-s_{*}+u_{L}\right)}{\left(s_{L}-u_{L}\right)} \\
& \frac{1}{2} \rho_{L}\left(s_{*}-u_{L}\right)^{2}-\frac{p_{L}+\bar{p}_{L}^{\infty}}{u_{L}-s_{L}}\left(s_{*}-u_{L}\right)+\left(\rho_{L} e_{L}-\bar{p}_{L}^{\infty}\right)>0 .
\end{aligned}
$$


Let $\beta=s_{*}-u_{L}$, then the inequality above is a quadratic function of $\beta$. We can show that this quadratic has no real roots by ensuring the discriminant is negative. That is,

$$
\left(\frac{p_{L}+\bar{p}_{L}^{\infty}}{u_{L}-s_{L}}\right)^{2}-2 \rho_{L}\left(\rho_{L} e_{L}-\bar{p}_{L}^{\infty}\right)<0 .
$$

This implies that we require:

$$
s_{L}<u_{L}-\frac{p_{L}+\bar{p}_{L}^{\infty}}{\sqrt{2 \rho_{L}\left(\rho_{L} e_{L}-\bar{p}_{L}^{\infty}\right)}} .
$$

It should be noted that:

$$
\begin{aligned}
\frac{p_{L}+\bar{p}_{L}^{\infty}}{\sqrt{2 \rho_{L}\left(\rho_{L} e_{L}-\bar{p}_{L}^{\infty}\right)}} & =\sqrt{\frac{\left(\bar{\gamma}_{L}-1\right)\left(p_{L}+\bar{p}_{L}^{\infty}\right)}{2 \rho_{L}}} \\
& <\sqrt{\frac{\bar{\gamma}_{L}\left(p_{L}+\bar{p}_{L}^{\infty}\right)}{\rho_{L}}} \\
& =c_{L} .
\end{aligned}
$$

Since $s_{L}=\min \left(\bar{u}-\bar{c}, u_{L}-c_{L}\right)$, the constraint on $s_{L}$ given by equation (57) is already satisfied and we have proved the solutions given by the left star state have positive partial densities and squared speed of sound. Besides, the volume fractions are bounded. Thus, the HLLC Riemann solver is positivity- and boundedness-preserving.

Equation (45) can be re-written as:

$$
\begin{aligned}
\mathbf{W}_{i, j}^{n+1}= & \sigma_{x}\left[\mathbf{W}_{i, j}^{n}+\lambda_{x}\left(\hat{\mathbf{G}}_{i-\frac{1}{2}, j}^{x,+}-\hat{\mathbf{G}}_{i+\frac{1}{2}, j}^{x,-}\right)\right] \\
& +\sigma_{y}\left[\mathbf{W}_{i, j}^{n}+\lambda_{y}\left(\hat{\mathbf{G}}_{i, j-\frac{1}{2}}^{y,+}-\hat{\mathbf{G}}_{i, j+\frac{1}{2}}^{y,-}\right)\right],
\end{aligned}
$$

where $\lambda_{x}=\Delta t /\left(\Delta x \sigma_{x}\right)$ and $\lambda_{y}=\Delta t /\left(\Delta y \sigma_{y}\right) . \quad \sigma_{x}$ and $\sigma_{y}$ are partitions of the contribution in the $x$ and $y$ directions respectively where $\sigma_{x}+\sigma_{y}=1$. They can be defined as [20]:

$$
\sigma_{x}=\frac{\tau_{x}}{\tau_{x}+\tau_{y}}, \quad \sigma_{y}=\frac{\tau_{y}}{\tau_{x}+\tau_{y}}, \quad \tau_{x}=\frac{(|u|+c)_{\max }}{\Delta x}, \quad \tau_{y}=\frac{(|v|+c)_{\max }}{\Delta y},
$$

such that $0<\sigma_{x}<1$ and $0<\sigma_{y}<1$. If the time-step size $\Delta t$ is given by a chosen CFL number, $C F L$, with the following equation:

$$
\Delta t=\frac{C F L}{\tau_{x}+\tau_{y}},
$$

one has the relations for the equivalent 1D time step sizes in different directions, $\Delta t^{x}$ and $\Delta t^{y}$ :

$$
\begin{aligned}
& \lambda_{x}=\frac{C F L}{(|u|+c)_{\max }}=\frac{\Delta t^{x}}{\Delta x}, \\
& \lambda_{y}=\frac{C F L}{(|v|+c)_{\max }}=\frac{\Delta t^{y}}{\Delta y} .
\end{aligned}
$$


We can define:

$$
\begin{aligned}
\mathbf{W}_{i, j}^{x} & =\mathbf{W}_{i, j}^{n}+\lambda_{x}\left(\hat{\mathbf{G}}_{i-\frac{1}{2}, j}^{x,+}-\hat{\mathbf{G}}_{i+\frac{1}{2}, j}^{x,-}\right), \\
\mathbf{W}_{i, j}^{y} & =\mathbf{W}_{i, j}^{n}+\lambda_{y}\left(\hat{\mathbf{G}}_{i, j-\frac{1}{2}}^{y,+}-\hat{\mathbf{G}}_{i, j+\frac{1}{2}}^{y,-}\right) .
\end{aligned}
$$

Since $\hat{\mathbf{G}}_{i \mp \frac{1}{2}, j}^{x, \pm}$ are obtained from the quasi-1D HLLC solutions in the $x$ direction, the approximate waves from the edges at $x_{i \pm \frac{1}{2}}$ do not interact if the CFL condition, $C F L \leq$ 0.5 , is satisfied (same for $\hat{\mathbf{G}}_{i, j \neq \frac{1}{2}}^{y, \pm}$ in the $y$ direction). Thus, equation (20) is satisfied for $\mathbf{W}_{i, j}^{x}$ (similar for $\mathbf{W}_{i, j}^{y}$ in $y$ direction). The first part of the RHS of equation (20) is the solution in the half cell from $x_{i-\frac{1}{2}}$ to $x_{i}$ at $t+\Delta t^{x}$ and the second part is the solution in another half cell. Using the finite volume approach on the left and right half cells, we will get:

$$
\begin{aligned}
\frac{1}{\Delta x} \int_{x_{i-\frac{1}{2}}}^{x_{i}} R\left(\frac{x-x_{i-\frac{1}{2}}}{\Delta t^{x}}, \mathbf{W}_{i-1, j}^{n}, \mathbf{W}_{i, j}^{n}\right) d x & =\frac{1}{2}\left[\mathbf{W}_{i, j}^{n}+2 \lambda_{x}\left(\hat{\mathbf{G}}_{i-\frac{1}{2}, j}^{x,+}-\mathbf{F}_{i, j}^{x}\right)\right], \\
\frac{1}{\Delta x} \int_{x_{i}}^{x_{i+\frac{1}{2}}} R\left(\frac{x-x_{i+\frac{1}{2}}}{\Delta t^{x}}, \mathbf{W}_{i, j}^{n}, \mathbf{W}_{i+1, j}^{n}\right) d x & =\frac{1}{2}\left[\mathbf{W}_{i, j}^{n}-2 \lambda_{x}\left(\hat{\mathbf{G}}_{i+\frac{1}{2}, j}^{x,-}-\mathbf{F}_{i, j}^{x}\right)\right] .
\end{aligned}
$$

If we define:

$$
\begin{aligned}
& \mathbf{W}_{i, j}^{x,-}=\frac{2}{\Delta x} \int_{x_{i-\frac{1}{2}}}^{x_{i}} R\left(\frac{x-x_{i-\frac{1}{2}}}{\Delta t}, \mathbf{W}_{i-1, j}^{n}, \mathbf{W}_{i, j}^{n}\right) d x \\
& \mathbf{W}_{i, j}^{x,+}=\frac{2}{\Delta x} \int_{x_{i}}^{x_{i+\frac{1}{2}}} R\left(\frac{x-x_{i+\frac{1}{2}}}{\Delta t}, \mathbf{W}_{i, j}^{n}, \mathbf{W}_{i+1, j}^{n}\right) d x
\end{aligned}
$$

we will get:

$$
\mathbf{W}_{i, j}^{x}=\frac{1}{2} \mathbf{W}_{i, j}^{x,-}+\frac{1}{2} \mathbf{W}_{i, j}^{x,+} .
$$

$\mathbf{W}_{i, j}^{x, \pm}$ are at physically admissible states since they are convex averaging of the approximate HLLC solutions. This also means $\mathbf{W}_{i, j}^{x}$ is also physically admissible. Equation (58) becomes:

$$
\mathbf{W}_{i, j}^{n+1}=\sigma_{x} \mathbf{W}_{i, j}^{x}+\sigma_{y} \mathbf{W}_{i, j}^{y} .
$$

Therefore, $\mathbf{W}_{i, j}^{n+1}$ is also physically admissible since it is a convex combination of $\mathbf{W}_{i, j}^{x}$ and $\mathbf{W}_{i, j}^{y}$.

\section{Incremental-stencil WCNS}

In this section, a high-order finite difference scheme for discretizing equation (28) is introduced. The high-order scheme belongs to the family of weighted compact nonlinear schemes (WCNSs) which is a variant of the WENO schemes for discontinuity-capturing. It was first proposed by Deng and Zhang [10] in which compact (spatially implicit) finite difference schemes are combined with WENO interpolation. Since then, WCNSs are extended to higher order of accuracy $[31,51]$. In principle, WCNSs can be used 
with both explicit or compact finite difference schemes. Nonomura and Fujii [29] suggested that explicit finite difference schemes are more efficient and later also proposed a family of robust explicit midpoint-and-node-to-node finite difference schemes [30]. In this section, an explicit WCNS with the explicit hybrid cell-midpoint and cell-node finite difference scheme $[9,50]$ and nonlinear interpolation adapted from incremental-stencil reconstruction [48] for finite volume WENO scheme is presented.

The semi-discretized finite difference form of equation (28) is given by:

$$
\left.\frac{\partial \mathbf{W}}{\partial t}\right|_{i, j}+\left.\frac{\widehat{\partial \mathbf{G}^{x}}}{\partial x}\right|_{i, j}+\left.\frac{\widehat{\partial \mathbf{G}^{y}}}{\partial y}\right|_{i, j}=\hat{\mathbf{S}}_{i, j}
$$

where $\hat{\mathbf{S}}_{i, j}$ is given by:

$$
\left.\hat{\mathbf{S}}_{i, j}=\left(\begin{array}{c}
0 \\
0 \\
0 \\
0 \\
0 \\
\alpha_{1, i, j}\left(\left.\frac{\widehat{\partial u}}{\partial x}\right|_{i, j}+\left.\frac{\widehat{\partial v}}{\partial y}\right|_{i, j}\right.
\end{array}\right)\right) .
$$

A high-order discretization is considered consistent (and conservative for any conservative equation) if:

$$
\begin{array}{rlrl}
\left.\frac{\partial \widehat{\mathbf{G}}^{x}}{\partial x}\right|_{i, j} & =\frac{\hat{\mathbf{G}}_{i+\frac{1}{2}, j}^{x}-\hat{\mathbf{G}}_{i-\frac{1}{2}, j}^{x}}{\Delta x}, & \left.\frac{\widehat{\partial \widehat{G}^{y}}}{\partial y}\right|_{i, j} & =\frac{\hat{\mathbf{G}}_{i, j+\frac{1}{2}}^{y}-\hat{\mathbf{G}}_{i, j-\frac{1}{2}}^{y}}{\Delta y}, \\
\left.\frac{\widehat{\partial u}}{\partial x}\right|_{i, j} & =\frac{\hat{u}_{i+\frac{1}{2}, j}-\hat{u}_{i-\frac{1}{2}, j}}{\Delta x}, & \left.\frac{\partial v}{\partial y}\right|_{i, j}=\frac{\hat{v}_{i, j+\frac{1}{2}}-\hat{v}_{i, j-\frac{1}{2}}}{\Delta y} .
\end{array}
$$

Therefore, equation (71) becomes:

$$
\left.\frac{\partial \mathbf{W}}{\partial t}\right|_{i, j}+\frac{\hat{\mathbf{G}}_{i+\frac{1}{2}, j}^{x}-\hat{\mathbf{G}}_{i-\frac{1}{2}, j}^{x}}{\Delta x}+\frac{\hat{\mathbf{G}}_{i, j+\frac{1}{2}}^{y}-\hat{\mathbf{G}}_{i, j-\frac{1}{2}}^{y}}{\Delta y}=\hat{\mathbf{S}}_{i, j},
$$

Equation (75) looks as same as equation (32) if it is further discretized in time with forward Euler method but $\hat{\mathbf{G}}_{i \pm \frac{1}{2}, j}^{x}, \hat{\mathbf{G}}_{i, j \pm \frac{1}{2}}^{y}$ and $\hat{\mathbf{S}}_{i, j}$ (composed of $\hat{u}_{i \pm \frac{1}{2}, j}$ and $\hat{v}_{i, j \pm \frac{1}{2}}$ ) are in high-order accurate approximations and are given by the WCNS introduced in this section. It should be noted that equation (75) is a conservative discretization for all conservative equations, except the last advection equation that is given by:

$$
\begin{array}{r}
\left.\frac{\partial \alpha_{1}}{\partial t}\right|_{i, j}+\frac{\hat{f}_{\alpha, i+\frac{1}{2}, j}^{x}-\hat{f}_{\alpha, i-\frac{1}{2}, j}^{x}+\frac{\hat{f}_{\alpha, i, j+\frac{1}{2}}^{y}-\hat{f}_{\alpha, i, j-\frac{1}{2}}^{y}}{\Delta x}=}{\Delta y}+ \\
\alpha_{1, i, j}\left(\frac{\hat{u}_{i+\frac{1}{2}, j}-\hat{u}_{i-\frac{1}{2}, j}}{\Delta x}+\frac{\hat{v}_{i, j+\frac{1}{2}}-\hat{v}_{i, j-\frac{1}{2}}}{\Delta y}\right) .
\end{array}
$$




\subsection{Explicit hybrid cell-midpoint and cell-node scheme}

The sixth order accurate explicit scheme from the hybrid cell-midpoint and cellnode compact scheme (HCS) [9] family is used for the approximation of the first order derivatives $\partial \mathbf{G}^{x} /\left.\partial x\right|_{i, j}$ and $\partial \mathbf{G}^{y} /\left.\partial y\right|_{i, j}$. The sixth order explicit HCS formulation is given by:

$$
\begin{aligned}
\left.\frac{\partial \mathbf{G}^{x}}{\partial x}\right|_{i, j} \approx & \left.\frac{\widehat{\partial \mathbf{G}^{x}}}{\partial x}\right|_{i, j} \\
= & \frac{1}{\Delta x}\left[\psi\left(\tilde{\mathbf{G}}_{i+\frac{1}{2}, j}^{x}-\tilde{\mathbf{G}}_{i-\frac{1}{2}, j}^{x}\right)-\frac{175 \psi-192}{256}\left(\mathbf{G}_{i+1, j}^{x}-\mathbf{G}_{i-1, j}^{x}\right)\right. \\
& \left.+\frac{35 \psi-48}{320}\left(\mathbf{G}_{i+2, j}^{x}-\mathbf{G}_{i-2, j}^{x}\right)-\frac{45 \psi-64}{3840}\left(\mathbf{G}_{i+3, j}^{x}-\mathbf{G}_{i-3, j}^{x}\right)\right] .
\end{aligned}
$$

If we replace $\tilde{\mathbf{G}}_{i \pm \frac{1}{2}, j}^{x}$ with the exact fluxes,

$$
\begin{aligned}
\left.\frac{\widehat{\partial \mathbf{G}^{x}}}{\partial x}\right|_{i, j}= & \left.\frac{\partial \mathbf{G}^{x}}{\partial x}\right|_{i, j}-\left.\left(\frac{5 \psi}{1024}-\frac{1}{140}\right) \frac{\partial^{7} \mathbf{G}^{x}}{\partial x^{7}}\right|_{i, j} \Delta x^{6} \\
& -\left.\left(\frac{95 \psi}{98304}-\frac{1}{720}\right) \frac{\partial^{9} \mathbf{G}^{x}}{\partial x^{9}}\right|_{i, j} \Delta x^{8}+\mathcal{O}\left(\Delta x^{10}\right) .
\end{aligned}
$$

If $\psi=256 / 175$, the scheme becomes eighth order accurate:

$$
\left.\frac{\widehat{\partial \mathbf{G}^{x}}}{\partial x}\right|_{i, j}=\left.\frac{\partial \mathbf{G}^{x}}{\partial x}\right|_{i, j}-\left.\frac{1}{40320} \frac{\partial^{9} \mathbf{G}^{x}}{\partial x^{9}}\right|_{i, j} \Delta x^{8}+\mathcal{O}\left(\Delta x^{10}\right) .
$$

$\psi=256 / 175$ is adopted in this work.

Any central explicit or compact finite difference scheme can be rewritten into the flux-difference forms given by equation (73) and it is derived in [44]. Following that work, the HCS given by equation (77) has implied reconstructed flux $\hat{\mathbf{G}}_{i+1 / 2, j}^{x}$ given by:

$$
\begin{aligned}
\hat{\mathbf{G}}_{i+\frac{1}{2}, j}^{x}= & \psi \tilde{\mathbf{G}}_{i+\frac{1}{2}, j}^{x}-\left(\frac{75 \psi}{128}-\frac{37}{60}\right)\left(\mathbf{G}_{i, j}^{x}+\mathbf{G}_{i+1, j}^{x}\right) \\
& +\left(\frac{25 \psi}{256}-\frac{2}{15}\right)\left(\mathbf{G}_{i-1, j}^{x}+\mathbf{G}_{i+2, j}^{x}\right)-\left(\frac{3 \psi}{256}-\frac{1}{60}\right)\left(\mathbf{G}_{i-2, j}^{x}+\mathbf{G}_{i+3, j}^{x}\right) .
\end{aligned}
$$

Note that the equation above is also used for reconstructing the flux $\hat{f}_{\alpha, i+\frac{1}{2}, j}^{x}$ in the advection equation.

High-order finite difference approximations of the velocity components are also required for $\hat{\mathbf{S}}_{i, j}$. Following the idea of [49], the numerical derivatives of the velocity components are also given by the same finite difference scheme as the flux derivatives:

$$
\begin{aligned}
&\left.\left.\frac{\partial u}{\partial x}\right|_{i, j} \approx \widehat{\partial u}\right|_{i, j}= \frac{1}{\Delta x}\left[\psi\left(\tilde{u}_{i+\frac{1}{2}, j}-\tilde{u}_{i-\frac{1}{2}, j}\right)-\frac{175 \psi-192}{256}\left(u_{i+1, j}-u_{i-1, j}\right)\right. \\
&\left.+\frac{35 \psi-48}{320}\left(u_{i+2, j}-u_{i-2, j}\right)-\frac{45 \psi-64}{3840}\left(u_{i+3, j}-u_{i-3, j}\right)\right] . \\
& 16
\end{aligned}
$$


The implied reconstructed velocity component $\hat{u}_{i+1 / 2, j}$ is given by:

$$
\begin{aligned}
\hat{u}_{i+\frac{1}{2}, j}= & \psi \tilde{u}_{i+\frac{1}{2}, j}-\left(\frac{75 \psi}{128}-\frac{37}{60}\right)\left(u_{i, j}+u_{i+1, j}\right)+\left(\frac{25 \psi}{256}-\frac{2}{15}\right)\left(u_{i-1, j}+u_{i+2, j}\right) \\
& -\left(\frac{3 \psi}{256}-\frac{1}{60}\right)\left(u_{i-2, j}+u_{i+3, j}\right) .
\end{aligned}
$$

The discretizations for flux and velocity component derivatives in $y$ direction are similar. High-order accurate approximations are required for $\tilde{\mathbf{G}}_{i+1 / 2, j}^{x}$ and $\tilde{u}_{i+1 / 2, j}$ to form high-order discretization for equation (75), which are discussed in the following sections. Finally, by using equation (44), equation (71) can be rewritten as:

$$
\left.\frac{\partial \mathbf{W}}{\partial t}\right|_{i}+\frac{\hat{\mathbf{G}}_{i+\frac{1}{2}, j}^{x,-}-\hat{\mathbf{G}}_{i-\frac{1}{2}, j}^{x,+}}{\Delta x}+\frac{\hat{\mathbf{G}}_{i, j+\frac{1}{2}}^{y,-}-\hat{\mathbf{G}}_{i, j-\frac{1}{2}}^{y,+}}{\Delta y}=\mathbf{0} .
$$

\subsection{Methodology of WCNS}

In WCNSs, the fluxes at the midpoints are obtained with aid of explicit nonlinear interpolations, which can also be interpreted as nonlinear filtering processes to avoid spurious oscillations near shocks and other discontinuities. For simplicity, the implementation details of a WCNS is explained with a 1D version of equation (83) in this and the following sub-section. Thus, superscript " $x$ " in $\mathbf{G}^{x}$ is dropped for convenience.

For the 1D five-equation model, the algorithm to obtain the high-order fluxes $\hat{\mathbf{G}}_{i+1 / 2}^{ \pm}$ with the WCNS approach is given below:

1. Convert all $\mathbf{W}_{i}$ in the stencils of left-biased and right-biased nonlinear WENO interpolations to primitive variable vectors $\mathbf{V}_{i}$.

2. Perform characteristic decomposition by transforming all $\mathbf{V}_{i}$ in the stencils of interpolations to characteristic variable vectors $\mathbf{U}_{i}$ with the projection matrix $\mathbf{R}_{i+1 / 2}^{-1}$ : $\mathbf{U}_{i}=\mathbf{R}_{i+1 / 2}^{-1} \mathbf{V}_{i}$

3. Compute $\tilde{\mathbf{U}}_{L}$ and $\tilde{\mathbf{U}}_{R}$ at each midpoint with $\mathbf{U}_{i}$ using left-biased and right-biased nonlinear WENO interpolations respectively.

4. Transform $\tilde{\mathbf{U}}_{L}$ and $\tilde{\mathbf{U}}_{R}$ to $\tilde{\mathbf{V}}_{L}$ and $\tilde{\mathbf{V}}_{R}$ with the projection matrix $\mathbf{R}_{i+1 / 2}: \tilde{\mathbf{V}}_{L}=$ $\mathbf{R}_{i+1 / 2} \tilde{\mathbf{U}}_{L}$, and $\tilde{\mathbf{V}}_{R}=\mathbf{R}_{i+1 / 2} \tilde{\mathbf{U}}_{R}$.

5. Convert $\tilde{\mathbf{V}}_{L}$ and $\tilde{\mathbf{V}}_{R}$ to $\tilde{\mathbf{W}}_{L}$ and $\tilde{\mathbf{W}}_{R}$.

6. Compute the high-order flux and velocity at each midpoint with the Riemann solver. If the HLLC Riemann solver is used: $\tilde{\mathbf{G}}_{i+\frac{1}{2}}=\mathbf{G}^{\mathrm{HLLC}}\left(\tilde{\mathbf{W}}_{L}, \tilde{\mathbf{W}}_{R}\right)$ and $\tilde{u}_{i+\frac{1}{2}}=u_{*}^{\mathrm{HLLC}}\left(\tilde{\mathbf{W}}_{L}, \tilde{\mathbf{W}}_{R}\right)$.

7. Compute the flux and velocity at the nodes: $\mathbf{G}_{i}=\mathbf{G}\left(\mathbf{W}_{i}\right)$ and $u_{i}=u\left(\mathbf{W}_{i}\right)$.

8. Reconstruct the flux $\hat{\mathbf{G}}_{i+\frac{1}{2}}$ and velocity $\hat{u}_{i+\frac{1}{2}}$ at the midpoints using the flux differencing approach (equations (80) and (82) in this work).

9. Compute $\hat{\mathbf{G}}_{i+1 / 2}^{ \pm}$using equation (44):

$$
\begin{aligned}
\hat{\mathbf{G}}_{i+\frac{1}{2}}^{-} & \left.=\hat{\mathbf{G}}_{i+\frac{1}{2}}-\alpha_{1, i} \quad \begin{array}{llllll}
0 & 0 & 0 & 0 & \hat{u}_{i+\frac{1}{2}}
\end{array}\right)^{T}, \\
\hat{\mathbf{G}}_{i+\frac{1}{2}}^{+} & =\hat{\mathbf{G}}_{i+\frac{1}{2}}-\alpha_{1, i+1}\left(\begin{array}{lllllll}
0 & 0 & 0 & 0 & \hat{u}_{i+\frac{1}{2}}
\end{array}\right)^{T} . \\
17 &
\end{aligned}
$$


In this work, only the incremental-stencil WENO interpolation of left-biased midpoint values is presented. The interpolation of right-biased midpoint values is similar due to symmetry and can be obtained by flipping the stencils and corresponding coefficients. The projection matrices for transformation between primitive variables and characteristic variables can be found in Appendix A. The projection matrices $\mathbf{R}_{i+1 / 2}$ are computed at midpoints $x_{i+1 / 2}$ with the arithmetic averages of partial densities, mixture density and speed of sound at $x_{i}$ and $x_{i+1}$.

\subsection{Incremental-stencil WENO interpolation}

The finite volume WENO scheme with the incremental-stencil reconstruction (WENOIS) was proposed by Wang et al. [48]. The finite volume WENO-IS is robust for compressible multi-phase problems with shocks and is also accurate for those problems due to high-order WENO reconstruction with the use of HLLC Riemann solver, which is well-known for its accuracy in capturing material interfaces. However, in general a finite volume WENO scheme is more expensive compared with finite difference WCNS and WENO schemes with similar orders of accuracy for multi-dimensional simulations. This is due to the fact that a finite volume scheme requires multi-dimensional reconstructions to obtain point values at many Gaussian points on the cell boundaries from cell averages when one desires third or high order of accuracy [41, 46, 8]. Generally, finite difference WENO schemes or WCNSs in explicit forms are four times cheaper in 2D and nine times cheaper in 3D compared to finite volume WENO schemes since multidimensional reconstructions are not required for the former schemes [36]. While the costs of WENO reconstruction in finite difference WENO schemes and WENO interpolation in finite difference WCNS methods are similar, a finite difference WENO scheme can only be used with flux-vector splitting methods, such as Lax-Friedrichs flux splitting, when high-order of accuracy is desired. The use of flux-difference splitting methods such as Riemann solvers in a finite difference WENO scheme for multi-dimensional simulations degenerates to only second order but a WCNS can still maintain high order of accuracy when used with a Riemann solver. Therefore, in this work we propose a WCNS with a WENO interpolation adapted from the robust incremental-stencil WENO reconstruction such that the HLLC Riemann solver can be applied for upwinding while the overall scheme is still high-order accurate and efficient.

The incremental-stencil (IS) interpolation approximates the midpoint values by nonlinear combination of linearly interpolated values from four different sub-stencils, $S_{0}-S_{3}$ (shown in figure 4). The interpolated values at the midpoints $\tilde{u}_{j+\frac{1}{2}}$ from the four different sub-stencils are given by:

$$
\begin{array}{ll}
S_{0}: & \tilde{u}_{i+\frac{1}{2}}^{0}=\frac{1}{2}\left(u_{i}+u_{i+1}\right), \\
S_{1}: & \tilde{u}_{i+\frac{1}{2}}^{1}=\frac{1}{2}\left(-u_{i-1}+3 u_{i}\right), \\
S_{2}: & \tilde{u}_{i+\frac{1}{2}}^{2}=\frac{1}{8}\left(3 u_{i}+6 u_{i+1}-u_{i+2}\right), \\
S_{3}: & \tilde{u}_{i+\frac{1}{2}}^{3}=\frac{1}{8}\left(3 u_{i-2}-10 u_{i-1}+15 u_{i}\right) .
\end{array}
$$

The interpolated values from stencils $S_{0}$ and $S_{1}$ are second order accurate and those from stencils $S_{2}$ and $S_{3}$ are third order accurate. The variable $u$ can either be fluxes, 
conservative variables, primitive variables or variables that are projected to the characteristic fields. In this work, the primitive variables projected to the characteristic fields are employed in the interpolation process.

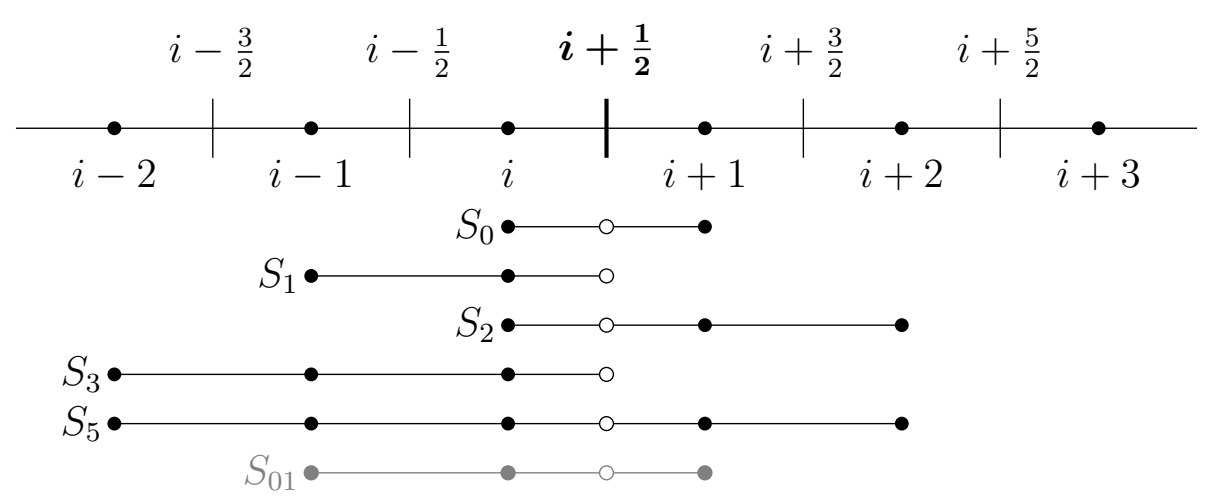

Figure 4: Sub-stencils of incremental-stencil WENO interpolation. The solid circles represent nodes used in the interpolation stencils, while empty circles represent the midpoint for the interpolated values. The stencil $S_{01}$ is in grey since it is not considered in the actual interpolation.

A fifth order 5-point linear interpolation from $S_{5}$ can be obtained from linear combination of the lower order interpolations:

$$
S_{5}: \quad \tilde{u}_{i+\frac{1}{2}}^{5}=\sum_{k=0}^{3} d_{k} \tilde{u}_{i+\frac{1}{2}}^{k}
$$

where the linear weights are given by:

$$
d_{0}=\frac{15}{32}, \quad d_{1}=\frac{5}{32}, \quad d_{2}=\frac{5}{16}, \quad d_{3}=\frac{1}{16} .
$$

The expanded form of the linear interpolation is given by:

$$
S_{5}: \quad \tilde{u}_{i+\frac{1}{2}}^{5}=\frac{1}{128}\left(3 u_{i-2}-20 u_{i-1}+90 u_{i}+60 u_{i+1}-5 u_{i+2}\right) .
$$

The 5-point linear interpolation scheme may generate spurious oscillations due to Gibbs phenomenon or is even unstable near shocks or discontinuities. To improve robustness, a nonlinear WENO interpolation is suggested in this work by replacing the linear weights with the incremental-stencil (IS) nonlinear weights [48]. The nonlinear weights have the following form:

$$
\tilde{u}_{i+\frac{1}{2}}=\sum_{k=0}^{3} \omega_{k} \tilde{u}_{i+\frac{1}{2}}^{k}
$$


The IS nonlinear weights are given by [48]:

$$
\begin{aligned}
\omega_{k} & =\frac{\eta_{k}}{\sum_{s=0}^{3} \eta_{s}}, \\
\eta_{k} & = \begin{cases}d_{k}\left(1+\frac{\tau_{5}}{\beta_{k}+\epsilon} \cdot \frac{\tau_{5}}{\beta_{01}+\epsilon}\right), & \text { if } k<2, \\
d_{k}\left(1+\frac{\tau_{5}}{\beta_{k}+\epsilon}\right), & \text { otherwise. }\end{cases}
\end{aligned}
$$

The smoothness indicators are defined by:

$$
\beta_{k}= \begin{cases}\int_{x_{i-\frac{1}{2}}}^{x_{i+\frac{1}{2}}} \Delta x\left(\frac{\partial}{\partial x} \tilde{u}^{k}(x)\right)^{2} d x, & k=0,1, \\ \sum_{l=1}^{2} \int_{x_{i-\frac{1}{2}}}^{x_{i+\frac{1}{2}}} \Delta x^{2 l-1}\left(\frac{\partial^{l}}{\partial x^{l}} \tilde{u}^{k}(x)\right)^{2} d x, & \text { otherwise (including } k=01),\end{cases}
$$

where $\tilde{u}^{k}(x)$ are the Lagrange interpolating polynomials from stencils $S_{k}$. The nonlinear weights require smoothness indicator computed with the Lagrange interpolation polynomial from stencil $S_{01}$ besides those from $S_{0}-S_{3}$. The integrated forms of smoothness indicators are given by:

$$
\begin{aligned}
& \beta_{0}=\left(u_{i}-u_{i+1}\right)^{2}, \\
& \beta_{1}=\left(u_{i-1}-u_{i}\right)^{2}, \\
& \beta_{01}=\frac{13}{12}\left(u_{i-1}-2 u_{i}+u_{i+1}\right)^{2}+\frac{1}{4}\left(u_{i-1}-u_{i+1}\right)^{2}, \\
& \beta_{2}=\frac{13}{12}\left(u_{i}-2 u_{i+1}+u_{i+2}\right)^{2}+\frac{1}{4}\left(3 u_{i}-4 u_{i+1}+u_{i+2}\right)^{2}, \\
& \beta_{3}=\frac{13}{12}\left(u_{i-2}-2 u_{i-1}+u_{i}\right)^{2}+\frac{1}{4}\left(u_{i-2}-4 u_{i-1}+3 u_{i}\right)^{2} .
\end{aligned}
$$

The reference smoothness indicator, $\tau_{5}$, is defined by [12]:

$$
\tau_{5}=\sum_{l=3}^{4} \int_{x_{i-\frac{1}{2}}}^{x_{i+\frac{1}{2}}} \Delta x^{2 l-1}\left(\frac{\partial^{l}}{\partial x^{l}} \tilde{l}^{5}(x)\right)^{2} d x
$$

where $\tilde{u}^{5}(x)$ is the Lagrange interpolating polynomial from stencil $S_{5}$. The integrated form of the reference smoothness indicator is given by:

$$
\begin{aligned}
\tau_{5}= & \frac{13}{12}\left(u_{i+2}-4 u_{i+1}+6 u_{i}-4 u_{i-1}+u_{i-2}\right)^{2} \\
& +\frac{1}{4}\left(u_{i+2}-2 u_{i+1}+2 u_{i-1}-u_{i-2}\right)^{2} .
\end{aligned}
$$

The robustness of incremental-stencil WENO interpolation presented above is as high as the WENO incremental-stencil reconstruction, as both of them can choose one of the 2-point stencils when there are closely located discontinuities, as explained in [48]. In Appendix B, it is proved that the IS nonlinear interpolation with the HCS finite differencing is fifth order accurate for a 1D scalar hyperbolic conservation law with perfect upwinding, provided not at critical points. This WCNS is termed WCNS-IS in this work. 


\section{Positivity- and boundedness-preserving limiting procedures}

Similar to the finite volume WENO-IS, the finite difference WCNS-IS is generally robust in capturing shocks and material interfaces in multi-phase flow simulations. However, it cannot be guaranteed that these high-order schemes are free from numerical failures due to negative squared speed of sound, partial densities (hence also out-ofbounds mass fractions), and out-of-bounds volume fractions. In this section, positivityand boundedness-preserving limiting procedures are introduced to improve the robustness of the WCNS-IS. The procedures are conservative for the corresponding equations in the system that are conservative, i.e. except the volume fraction advection equation.

In the algorithm of WCNS, there are two stages where positivity and boundedness can be violated. The first stage is the WENO interpolation step for the left-biased and right-biased interpolated conservative variable vectors $\left(\tilde{\mathbf{W}}_{L}\right.$ and $\left.\tilde{\mathbf{W}}_{R}\right)$, where the interpolated conservative variables may not be physically admissible. Another stage is the flux reconstruction step of $\hat{\mathbf{G}}_{i+1 / 2}^{ \pm}$using the Riemann solver and the high-order HCS finite difference scheme. The positivity- and boundedness-preserving interpolation limiter and flux limiter are introduced in this section to respectively deal with the two issues mentioned.

\subsection{Positivity- and boundedness-preserving interpolation limiter}

The incremental-stencil WENO interpolation is robust but it is still not positivitypreserving for partial densities and squared speed of sound. It is also not boundednesspreserving for volume fractions. However, the first order interpolation with the left and right node values are in the admissible state set and are positivity-preserving and boundedness-preserving. Therefore, the high-order WENO interpolation can be limited with a convex combination of itself and the first order interpolation. For simplicity, this sub-section only discusses the limiting procedures for the left-biased WENO interpolation in the $x$ direction for the governing equations. Hence, the subscripts " $L$ " and " $j$ " are omitted.

The first stage of the interpolation limiting procedures is to obtain a limited conservative variable vector with positive partial densities at each midpoint, $\tilde{\mathbf{W}}_{i+\frac{1}{2}}^{*}$. As described in algorithm $1, \tilde{\mathbf{W}}_{i+\frac{1}{2}}^{*}$ is first initialized as the WENO interpolated conservative variable vector. It is then limited for positive partial densities through repeated convex combination of $\tilde{\mathbf{W}}_{i+\frac{1}{2}}^{*}$ with the first order interpolated conservative variable vector for each phase using a user-defined small tolerance $\epsilon_{\alpha_{k} \rho_{k}}$. In the next stage, limiting procedure on the volume fractions can be applied similarly through another set of successive convex combinations given by algorithm 2 with another user-defined small threshold $\epsilon_{\alpha_{k}}$. After this stage, the limited vector $\tilde{\mathbf{W}}_{i+\frac{1}{2}}^{* *}$ should have all partial densities (including mixture density) positive and all volume fractions bounded between $\epsilon_{\alpha_{k}}$ and $1-\epsilon_{\alpha_{k}}$. This also means that all mass fractions are bounded between 0 and 1 . The remaining quantity to limit is the squared speed of sound.

We can define a helper variable $\tilde{c}$ where $\tilde{c}^{2}=\left(\rho e-\bar{p}^{\infty}\right) / \rho$ such that squared sound speed and $\tilde{c}$ are related through $c^{2}=\bar{\gamma}(\bar{\gamma}-1) \tilde{c}^{2}$. As the final stage of the limiting procedures, $\tilde{\mathbf{W}}_{i+\frac{1}{2}}^{* * *}$ with positive $\rho \tilde{c}^{2}$ can be obtained using algorithm 3 . This is carried out through convex combination of $\tilde{\mathbf{W}}_{i+\frac{1}{2}}^{* *}$ and the first order interpolated conservative 
variable vector with tolerance $\epsilon_{\rho \tilde{c}^{2}}$ by utilizing the convexity of the admissible set proved with the Jensen's inequality. The squared speed of sound of the final limited conservative variable vector, $\tilde{\mathbf{W}}_{i+\frac{1}{2}}^{* * *}$, is also positive as $c^{2}=\bar{\gamma}(\bar{\gamma}-1)\left(\rho \tilde{c}^{2}\right) / \rho$. Note that $\bar{\gamma}$ is larger than 1 since volume fractions of $\tilde{\mathbf{W}}_{i+\frac{1}{2}}^{* *}$ are already bounded between 0 and 1 . The positivity and boundedness limiting procedures for the right-biased WENO interpolation in the $x$ direction can be performed with $\mathbf{W}_{i+1}$ instead of $\mathbf{W}_{i}$ in a similar way. We have chosen the tolerances for the limiting procedures as $\epsilon_{\alpha_{k} \rho_{k}}=1.0 \mathrm{e}-10, \epsilon_{\alpha_{k}}=1.0 \mathrm{e}-10$, and $\epsilon_{\rho \tilde{c}^{2}}=1.0 \mathrm{e}-8$. Note that in practice, user should make sure the tolerances are chosen to be smaller than the minimum values of the corresponding initial fields.

Due to numerical round-off, the $\tilde{\mathbf{W}}_{i+\frac{1}{2}}^{* * *}$ may still have negative partial densities, negative squared speed of sound, or out-of-bounds volume fractions. Therefore, a hard switch is suggested by setting $\tilde{\mathbf{W}}_{i+\frac{1}{2}}^{* * *}=\mathbf{W}_{i}$ when $\alpha_{k} \rho_{k}\left(\tilde{\mathbf{W}}_{i+\frac{1}{2}}^{* * *}\right)<\epsilon_{\alpha_{k} \rho_{k}}^{\mathrm{HS}}, \alpha_{k}\left(\tilde{\mathbf{W}}_{i+\frac{1}{2}}^{* * *}\right)<\epsilon_{\alpha_{k}}^{\mathrm{HS}}$, or $\rho \tilde{c}^{2}\left(\tilde{\mathbf{W}}_{i+\frac{1}{2}}^{* * *}\right)<\epsilon_{\rho \tilde{c}^{2}}^{\mathrm{HS}}$. The tolerances of the hard switch are chosen as $\epsilon_{\alpha_{k} \rho_{k}}^{\mathrm{HS}}=1.0 \mathrm{e}-11$, $\epsilon_{\alpha_{k}}^{\mathrm{HS}}=1.0 \mathrm{e}-11$, and $\epsilon_{\rho \tilde{c}^{2}}^{\mathrm{HS}}=1.0 \mathrm{e}-9$.

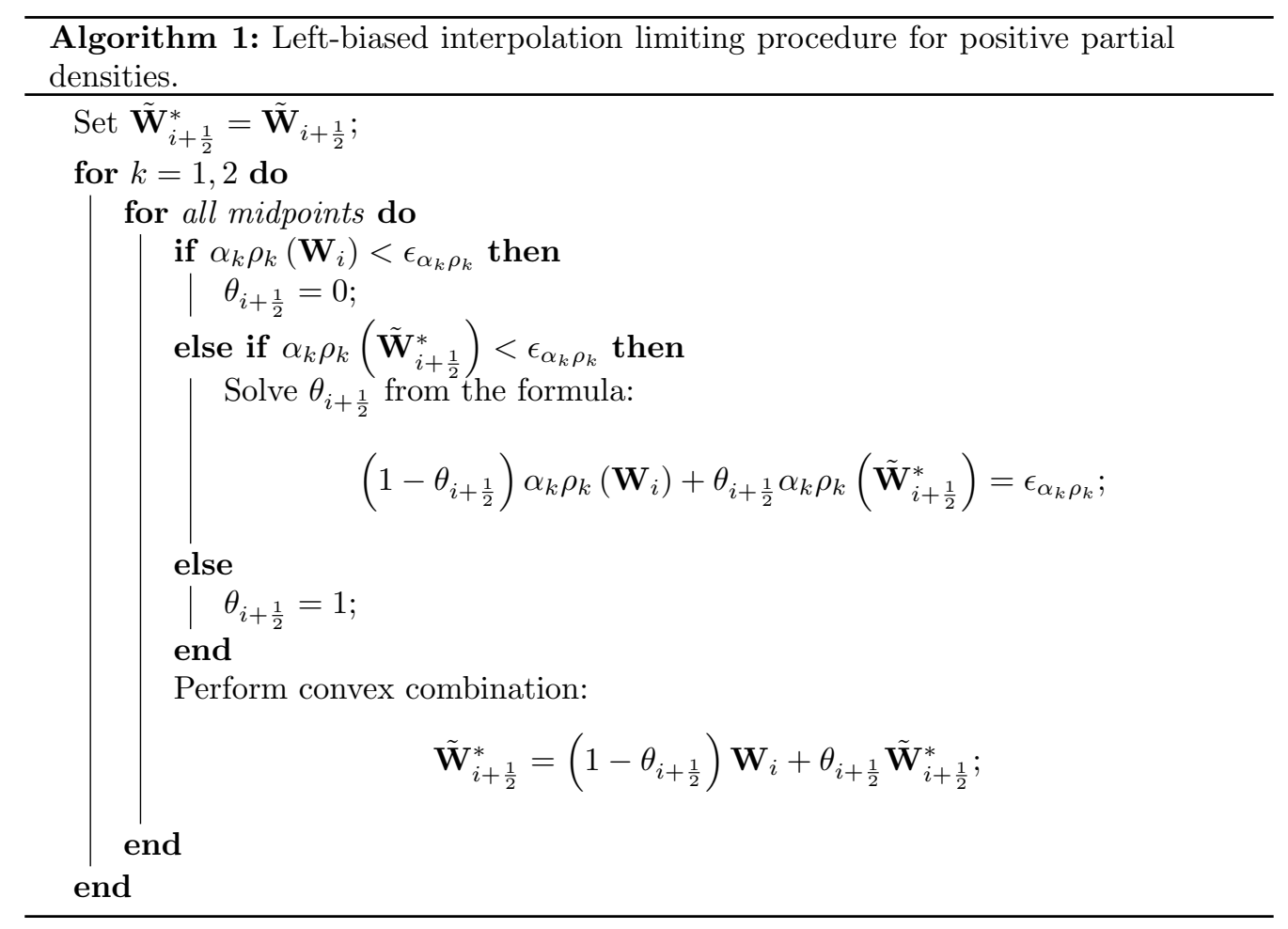

\subsection{Positivity- and boundedness-preserving flux limiter}

The high-order flux reconstruction step of $\hat{\mathbf{G}}_{i+1 / 2}^{ \pm}$using a Riemann solver and the HCS finite difference scheme is not positivity- and boundedness-preserving in general and may cause numerical failures. Therefore, a flux limiter is critical to make sure that 

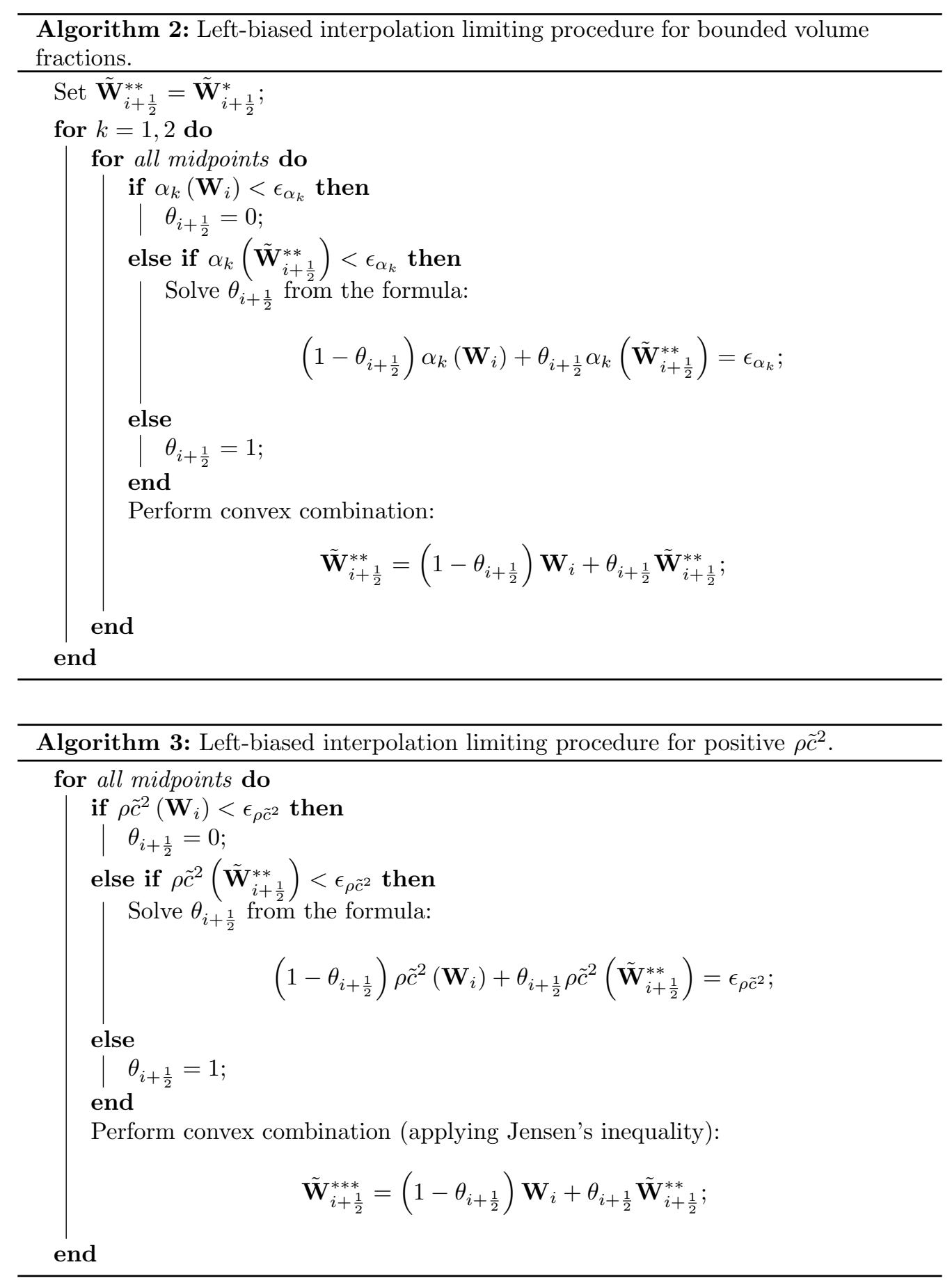
the flux used for time stepping gives physically admissible state. Following the same splitting idea introduced in the section of HLLC Riemann solver, if first order forward Euler time stepping is used for the WCNS, equation (83) can be rewritten as:

$$
\mathbf{W}_{i, j}^{n+1}=\sigma_{x} \underbrace{\left(\frac{1}{2} \mathbf{W}_{i, j}^{x,-}+\frac{1}{2} \mathbf{W}_{i, j}^{x,+}\right)}_{\mathbf{W}_{i, j}^{x}}+\sigma_{y} \underbrace{\left(\frac{1}{2} \mathbf{W}_{i, j}^{y,-}+\frac{1}{2} \mathbf{W}_{i, j}^{y,+}\right)}_{\mathbf{W}_{i, j}^{y}},
$$

where

$$
\begin{aligned}
& \mathbf{W}_{i, j}^{x, \mp}=\mathbf{W}_{i, j}^{n} \pm 2 \lambda_{x}\left(\hat{\mathbf{G}}_{i \mp \frac{1}{2}, j}^{x, \pm}-\mathbf{F}_{i, j}^{x}\right), \\
& \mathbf{W}_{i, j}^{y, \mp}=\mathbf{W}_{i, j}^{n} \pm 2 \lambda_{y}\left(\hat{\mathbf{G}}_{i, j \mp \frac{1}{2}}^{y, \pm}-\mathbf{F}_{i, j}^{y}\right) .
\end{aligned}
$$

Note that $\hat{\mathbf{G}}_{i \mp \frac{1}{2}, j}^{x, \pm}$ and $\hat{\mathbf{G}}_{i, j \mp \frac{1}{2}}^{y, \pm}$ here are the high-order reconstructed fluxes in contrast to the first order HLLC fluxes, $\hat{\mathbf{G}}_{i \mp \frac{1}{2}, j}^{x, \mathrm{HLLC}, \pm}$ and $\hat{\mathbf{G}}_{i, j \mp \frac{1}{2}}^{y, \mathrm{HLLC} \pm}$. Since both $\sigma_{x}$ and $\sigma_{y}$ are positive and $\sigma_{x}+\sigma_{y}=1, \mathbf{W}_{i, j}^{n+1}$ is a convex combination of $\mathbf{W}_{i, j}^{x, \pm}$ and $\mathbf{W}_{i, j}^{y, \pm}$. If all four conservative variable vectors are in the physically admissible set, $\mathbf{W}_{i, j}^{n+1}$ is also in the physically admissible set. For simplicity, only positivity- and boundedness-preserving flux limiting in $x$ direction for $\mathbf{W}_{i, j}^{x, \pm}$ is discussed here and hence " $x$ " superscript and " $j$ " subscript are dropped in the following part.

If the first order flux from the approximate Riemann solver is positively preserving such that all intermediate states given by the approximate Riemann solutions are in the admissible state set, such as the HLLC Riemann solver presented in this work, we can first construct the positivity flux limiting procedure for partial densities through the convex combination of the high-order solution $\mathbf{W}_{i}^{ \pm}$and the first order solution $\mathbf{W}_{i}^{\mathrm{HLLC}, \pm}$. This first stage of the flux limiting procedures to obtain the limited flux $\hat{\mathbf{G}}_{i+\frac{1}{2}}^{*, \pm}$ is detailed in algorithm 4 with tolerance $\epsilon_{\alpha_{k} \rho_{k}}$. After this stage, both solutions $\mathbf{W}_{i}^{*, \pm}$ time-advanced with the limited fluxes have all partial densities (including mixture density) positive, where

$$
\mathbf{W}_{i}^{*, \mp}=\mathbf{W}_{i}^{n} \pm 2 \lambda\left(\hat{\mathbf{G}}_{i \mp \frac{1}{2}}^{*, \pm}-\mathbf{F}_{i}\right) .
$$

Note that in the last step of the algorithm, the intention is to hybridize $\left(\hat{\mathbf{G}}_{i+\frac{1}{2}}^{*, \pm}-\mathbf{F}_{i}\right)$ with $\left(\hat{\mathbf{G}}_{i+\frac{1}{2}}^{\text {HLLC } \pm}-\mathbf{F}_{i}\right)$ but the $\mathbf{F}_{i}$ on both sides of the equation cancel each other. Also, the flux limiting process is conservative for all equations except the last advection equation of volume fraction.

In the next stage, we can apply the boundedness flux limiting for volume fractions similarly to obtain the limited flux $\hat{\mathbf{G}}_{i+\frac{1}{2}}^{* *, \pm}$, which is given in algorithm 5 with threshold $\epsilon_{\alpha_{k}}$. After this step, the limited solutions $\mathbf{W}_{i}^{* *, \pm}$ should have all volume fractions bounded in addition to positive partial densities (including mixture density), where

$$
\mathbf{W}_{i}^{* *, \mp}=\mathbf{W}_{i}^{n} \pm 2 \lambda\left(\hat{\mathbf{G}}_{i \mp \frac{1}{2}}^{* *, \pm}-\mathbf{F}_{i}\right)
$$

Finally, the positivity flux limiting for squared sound speed is conducted through the helper variable $\rho \tilde{c}^{2}$ similarly with the previous sub-section. This step is described 
in algorithm 6 with tolerance $\epsilon_{\rho \tilde{c}^{2}}$ and makes use of the fact that the admissible set of the conservative variable vector is convex to obtain the final limited flux $\hat{\mathbf{G}}_{i+\frac{1}{2}}^{* * * \pm}$. The squared speeds of sound $c^{2}$ of $\mathbf{W}_{i}^{* * *, \pm}$, where

$$
\mathbf{W}_{i}^{* * *, \mp}=\mathbf{W}_{i}^{n} \pm 2 \lambda\left(\hat{\mathbf{G}}_{i \mp \frac{1}{2}}^{* * * \pm}-\mathbf{F}_{i}\right),
$$

are limited to be positive since $c^{2}=\bar{\gamma}(\bar{\gamma}-1)\left(\rho \tilde{c}^{2}\right) / \rho$.

Note that the tolerance values $\left(\epsilon_{\alpha_{k} \rho_{k}}, \epsilon_{\alpha_{k}}\right.$, and $\left.\epsilon_{\rho \tilde{c}^{2}}\right)$ are as same as those used in the positivity- and boundedness-preserving interpolation limiter. Similar to the interpolation limiter, a hard switch is used by setting $\hat{\mathbf{G}}_{i+\frac{1}{2}}^{* * * \pm}=\hat{\mathbf{G}}_{i+\frac{1}{2}}^{\mathrm{HLLC}, \pm}$ if either $\mathbf{W}_{i}^{* * *,+}$ or $\mathbf{W}_{i+1}^{* * *-}$ are at states that are not bounded by smaller tolerances $\left(\epsilon_{\alpha_{k} \rho_{k}}^{\mathrm{HS}}=1.0 \mathrm{e}-11\right.$, $\epsilon_{\alpha_{k}}^{\mathrm{HS}}=1.0 \mathrm{e}-11$, and $\left.\epsilon_{\rho \tilde{c}^{2}}^{\mathrm{HS}}=1.0 \mathrm{e}-9\right)$.

\subsection{Extension to strong stability preserving Runge-Kutta time stepping scheme}

The extension of positivity- and boundedness-preserving limiters for high-order flux with the high-order strong stability preserving Runge-Kutta (SSPRK) time stepping methods $[40,14,13]$ is trivial since SSPRK time stepping schemes are convex combinations of Euler forward steps. However, the upper limit of CFL number is still constrained by 0.5 .

\section{Test problems}

1D and 2D test problems are conducted with the first order HLLC scheme and the high-order WCNS-IS with the positivity- and boundedness-preserving limiters. The two schemes are termed HLLC and PP-WCNS-IS respectively in this work. All tests involve liquid water and air as an ideal gas. The properties of the fluids are given in table 1. Note that the fluids satisfy the requirement from the positivity- and boundedness-preserving limiters as the ratio of specific heats of water is larger than that of air. The three-stage third order SSPRK scheme (TVDRK3) [40] is used for time stepping for both schemes. The CFL number is chosen to be 0.5 unless constant time step size is used. When constant time step size is used, the corresponding CFL number is always less than 0.5 until the end of simulations.

\subsection{Convergence study}

To verify the formal order of accuracy of each scheme, advection of volume fraction disturbance in a 2D periodic domain $[-1,1) \mathrm{m} \times[-1,1) \mathrm{m}$ is used as the test problem similar to that in [49]. The initial conditions are given by table 2 and the exact solutions are given by table 3 .

Simulations using different schemes are conducted up to $t=0.1 \mathrm{~ms}$ with mesh refinements from $N_{x}=N_{y}=8$ to $N_{x}=N_{y}=256$. All simulations are run with very small constant time steps in order to observe the spatial orders of accuracy of different numerical schemes. $\Delta t / \Delta x=4.0 e-5 \mathrm{~s} \mathrm{~m}^{-1}$ is used. 


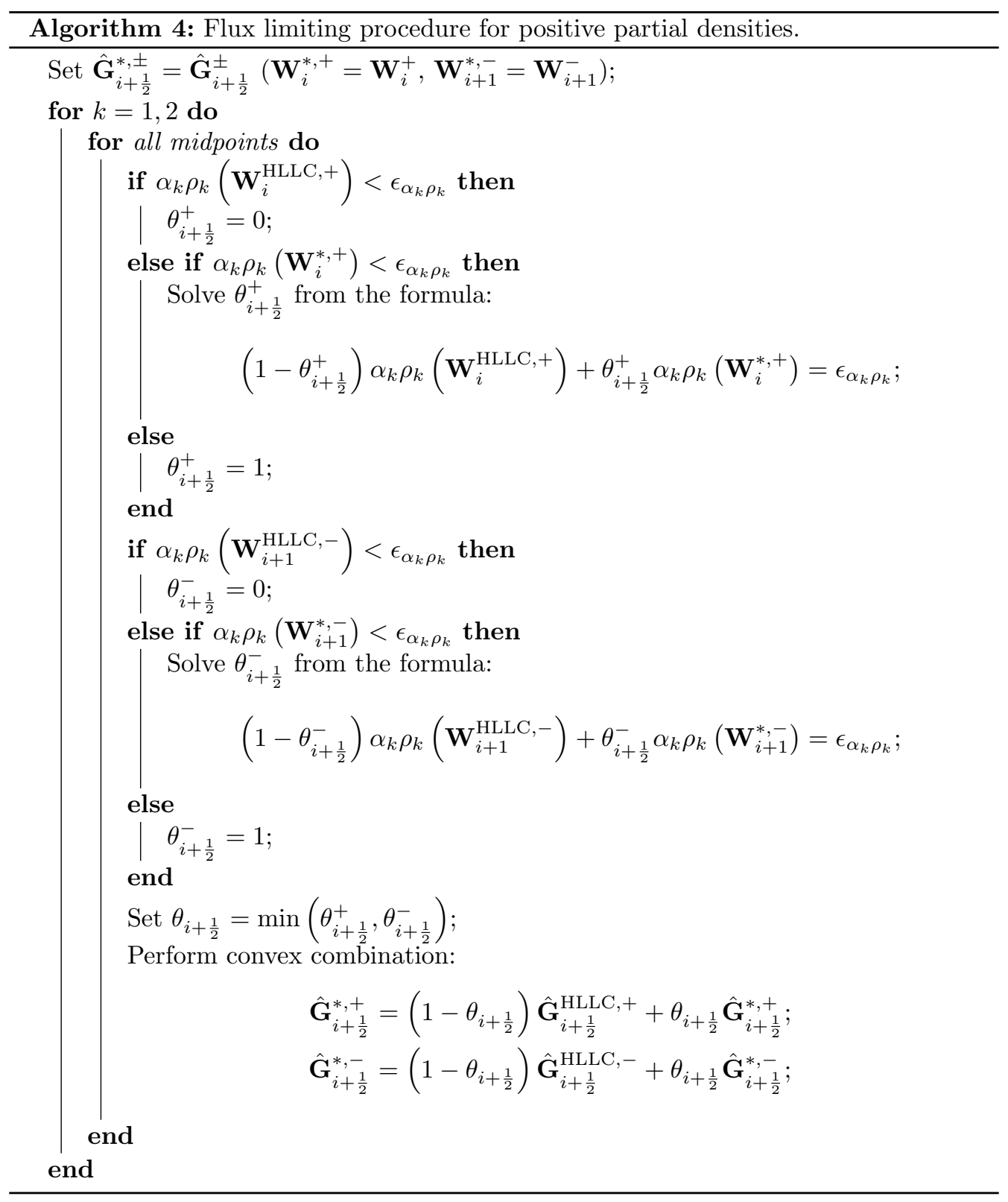

Table 4 shows the $L_{2}$ errors and the computed rates of convergence of volume fraction $\alpha_{1}$ respectively by the two schemes at $t=0.1 \mathrm{~ms}$. The $L_{2}$ error of volume fraction is 


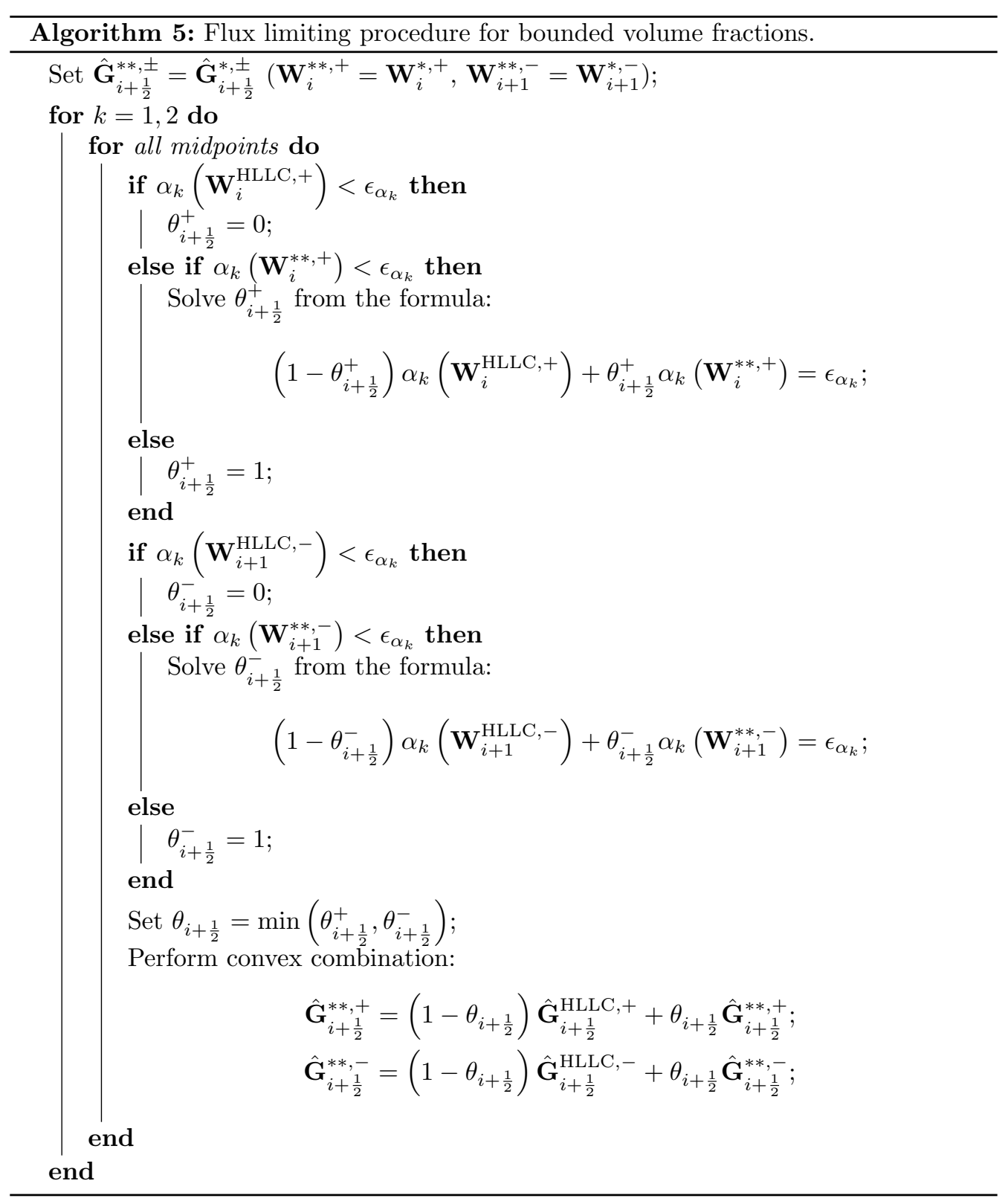

computed as:

$$
L_{2} \text { error }=\sqrt{\sum_{i=0}^{N-1} \sum_{j=0}^{N-1} \Delta x \Delta y\left(\alpha_{1 i, j}-\alpha_{1, j}^{\text {exact }}\right)^{2} / \sum_{i=0}^{N-1} \sum_{j=0}^{N-1} \Delta x \Delta y}
$$

where $\alpha_{1}{ }_{i, j}^{\text {exact }}$ is the exact solution of volume fraction at the corresponding grid point. It 


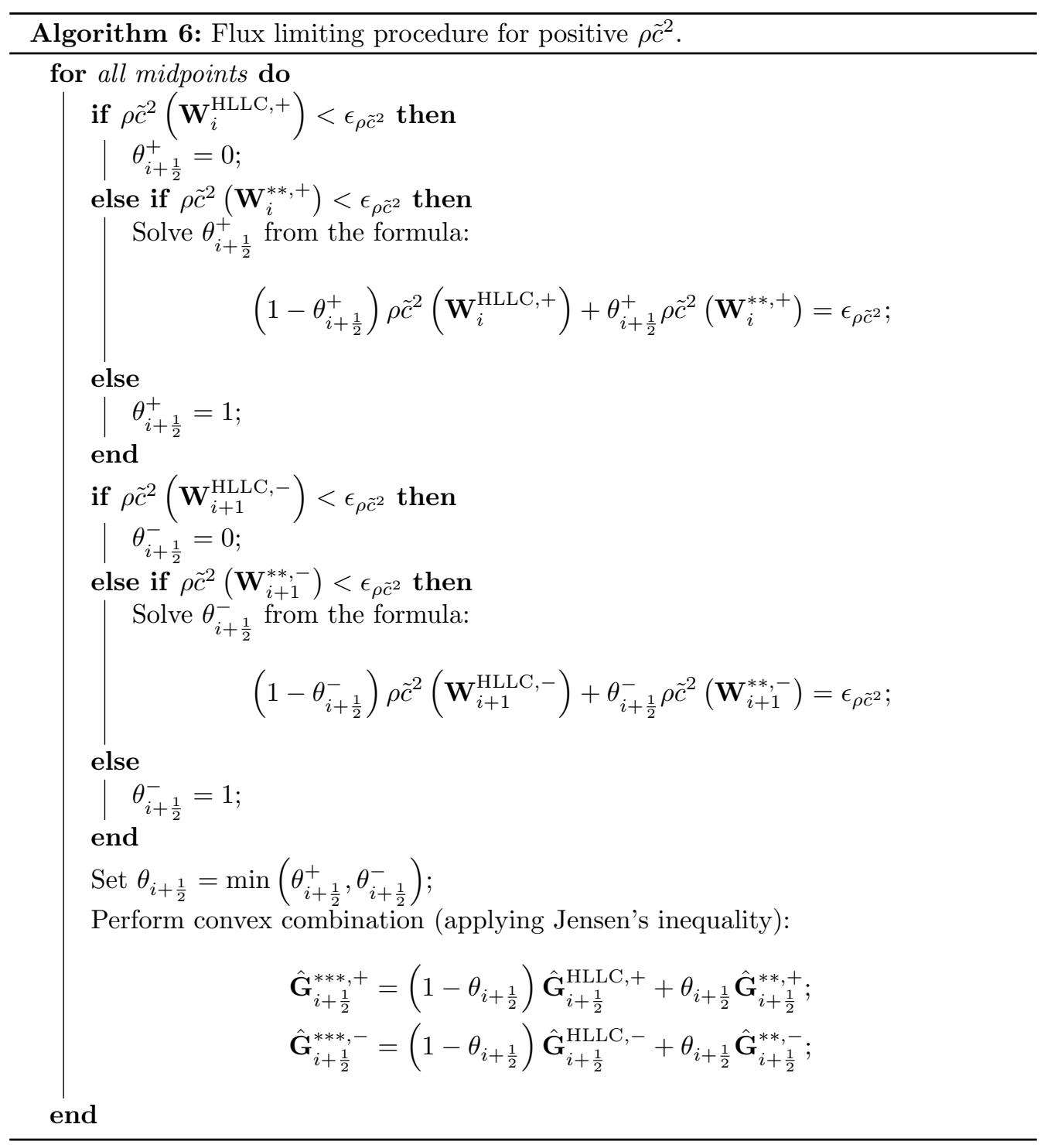

\begin{tabular}{c|ccc}
\hline Fluid & Phase number & $\gamma$ & $p_{\infty}(\mathrm{Pa})$ \\
\hline Liquid water & 1 & 6.12 & $3.43 \mathrm{e} 8$ \\
Air & 2 & 1.40 & 0 \\
\hline
\end{tabular}

Table 1: Properties of the fluids.

can be seen from the table that all schemes achieve the expected rates of convergence. 


\begin{tabular}{cccccc}
\hline $\begin{array}{c}\rho_{1} \\
\left(\mathrm{~kg} \mathrm{~m}^{-3}\right)\end{array}$ & $\begin{array}{c}\rho_{2} \\
\left(\mathrm{~kg} \mathrm{~m}^{-3}\right)\end{array}$ & $\begin{array}{c}u \\
\left(\mathrm{~m} \mathrm{~s}^{-1}\right)\end{array}$ & $\begin{array}{c}v \\
\left(\mathrm{~m} \mathrm{~s}^{-1}\right)\end{array}$ & $\begin{array}{c}p \\
(\mathrm{~Pa})\end{array}$ & $\alpha_{1}$ \\
\hline 1000 & 1 & 10 & 10 & 101325 & $0.5+0.25 \sin [\pi(x+y)]$ \\
\hline
\end{tabular}

Table 2: Initial conditions of 2D convergence problem.

\begin{tabular}{cccccc}
\hline $\begin{array}{c}\rho_{1} \\
\left(\mathrm{~kg} \mathrm{~m}^{-3}\right)\end{array}$ & $\begin{array}{c}\rho_{2} \\
\left(\mathrm{~kg} \mathrm{~m}^{-3}\right)\end{array}$ & $\begin{array}{c}u \\
\left(\mathrm{~m} \mathrm{~s}^{-1}\right)\end{array}$ & $\begin{array}{c}v \\
\left(\mathrm{~m} \mathrm{~s}^{-1}\right)\end{array}$ & $\begin{array}{c}p \\
(\mathrm{~Pa})\end{array}$ & $\alpha_{1}$ \\
\hline 1000 & 1 & 10 & 10 & 101325 & $0.5+0.25 \sin [\pi(x+y-20 t)]$ \\
\hline
\end{tabular}

Table 3: Exact solutions of 2D convergence problem.

\begin{tabular}{c|cccc}
\hline \multirow{2}{*}{$\begin{array}{c}\text { Number of } \\
\text { grid points }\end{array}$} & HLLC & \multicolumn{2}{c}{ PP-WCNS-IS } \\
\cline { 2 - 5 } & error & order & error & order \\
\hline $8^{2}$ & $4.283 \mathrm{e}-04$ & & $3.136 \mathrm{e}-05$ & \\
$16^{2}$ & $2.170 \mathrm{e}-04$ & 0.98 & $1.517 \mathrm{e}-07$ & 7.69 \\
$32^{2}$ & $1.089 \mathrm{e}-04$ & 0.99 & $4.855 \mathrm{e}-09$ & 4.97 \\
$64^{2}$ & $5.450 \mathrm{e}-05$ & 1.00 & $1.679 \mathrm{e}-10$ & 4.85 \\
$128^{2}$ & $2.726 \mathrm{e}-05$ & 1.00 & $5.382 \mathrm{e}-12$ & 4.96 \\
$256^{2}$ & $1.363 \mathrm{e}-05$ & 1.00 & $1.714 \mathrm{e}-13$ & 4.97 \\
\hline
\end{tabular}

Table 4: $L_{2}$ errors and orders of convergence of volume fraction for the $2 \mathrm{D}$ advection problem from different schemes at $t=0.1 \mathrm{~ms}$.

\subsection{One-dimensional material interface advection}

The next multi-phase problem is a $1 \mathrm{D}$ problem with the advection of two material interfaces. The settings of this problem are similar to those in $[8,49,2]$. The initial conditions are given by table 5 . Periodic conditions are applied at both boundaries. The spatial domain is $x \in[0,1) \mathrm{m}$ and the final time is at $t=0.01 \mathrm{~s}$. Simulations are evolved with constant time steps $\Delta t=1.25 \mathrm{e}-6 \mathrm{~s}$ on a uniform grid with 200 grid points where $\Delta x=0.005 \mathrm{~m}$. The two material interfaces have exactly advected one period at the end of the simulations.

\begin{tabular}{c|ccccc}
\hline & $\begin{array}{c}\alpha_{1} \rho_{1} \\
\left(\mathrm{~kg} \mathrm{~m}^{-3}\right)\end{array}$ & $\begin{array}{c}\alpha_{2} \rho_{2} \\
\left(\mathrm{~kg} \mathrm{~m}^{-3}\right)\end{array}$ & $\begin{array}{c}u \\
\left(\mathrm{~m} \mathrm{~s}^{-1}\right)\end{array}$ & $\begin{array}{c}p \\
(\mathrm{~Pa})\end{array}$ & $\alpha_{1}$ \\
\hline $\begin{array}{c}0.25 \leq x<0.75 \\
\text { otherwise }\end{array}$ & 1000 & $1.0 \mathrm{e}-8$ & 100 & 101325 & $1-1.0 \mathrm{e}-8$ \\
\hline
\end{tabular}

Table 5: Initial conditions of 1D material interface advection problem.

The density fields obtained with the two schemes at the final simulation time are compared with the exact solution in figure 5. It can be seen that the high-order PP-WCNS-IS 
can capture the material interfaces with much smaller numerical widths compared to the first order HLLC scheme and no spurious oscillations are observed at the two material interfaces for PP-WCNS-IS. Both the velocity and pressure fields are uniform and constant in this advection problem. In figure 6 , it can be seen that the relative errors in velocity and pressure fields for both schemes are insignificantly small and the uniform and constant fields are maintained well over time.

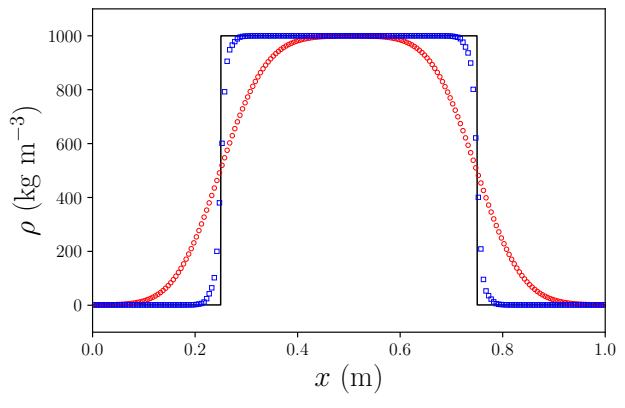

(a) Global density profile

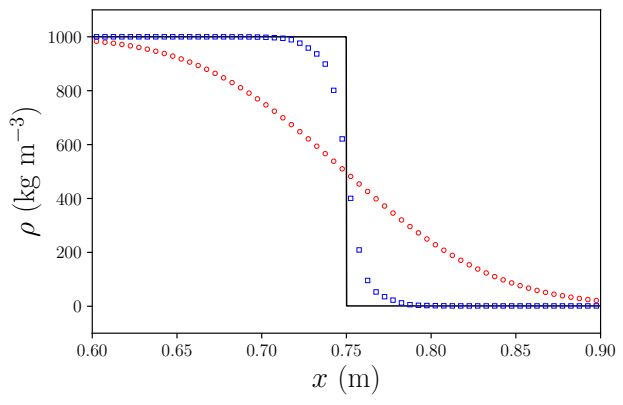

(b) Local density profile

Figure 5: Material interface advection problem at $t=0.01 \mathrm{~s}$ using different schemes. Black solid line: exact; red circles: HLLC; blue squares: PP-WCNS-IS.

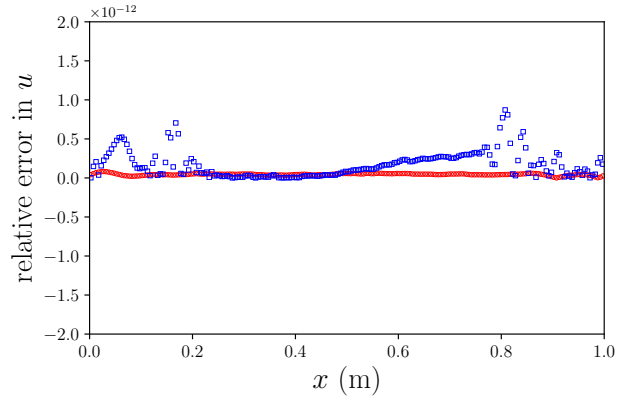

(a) Velocity

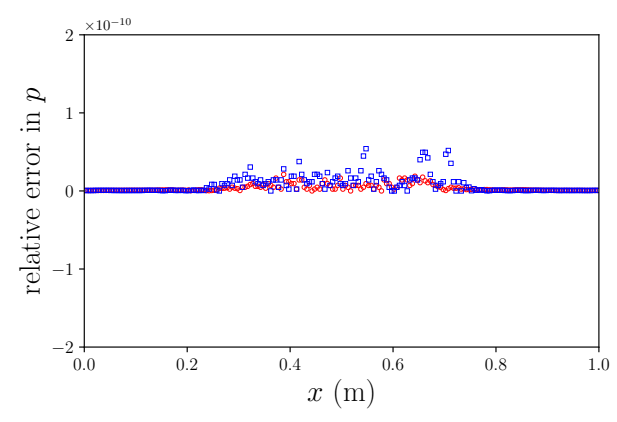

(b) Pressure

Figure 6: Relative errors for the material interface advection problem at $t=0.01 \mathrm{~s}$ using different schemes. Red circles: HLLC; blue squares: PP-WCNS-IS.

\subsection{One-dimensional gas/liquid Sod shock tube problem}

This gas/liquid shock tube problem is taken from Chen and Liang [6] and Wang et al. [48]. The initial conditions are given by table 6. Extrapolations are applied at both boundaries. The spatial domain is $x \in[0,1.5] \mathrm{m}$ and the final time is at $t=3 \mathrm{e}-4 \mathrm{~s}$. Simulations are evolved with constant time steps $\Delta t=1.25 \mathrm{e}-6 \mathrm{~s}$ on a uniform grid with 200 grid points. The reference solutions are obtained using PP-WCNS-IS with 4000 grid points. 


\begin{tabular}{l|ccccc}
\hline & $\begin{array}{c}\alpha_{1} \rho_{1} \\
\left(\mathrm{~kg} \mathrm{~m}^{-3}\right)\end{array}$ & $\begin{array}{c}\alpha_{2} \rho_{2} \\
\left(\mathrm{~kg} \mathrm{~m}^{-3}\right)\end{array}$ & $\begin{array}{c}u \\
\left(\mathrm{~m} \mathrm{~s}^{-1}\right)\end{array}$ & $\begin{array}{c}p \\
(\mathrm{~Pa})\end{array}$ & $\alpha_{1}$ \\
\hline$x<0.8$ & 1000 & $1.0 \mathrm{e}-8$ & 0 & $1.0 \mathrm{e} 9$ & $1-1.0 \mathrm{e}-8$ \\
$x \geq 0.8$ & $1.0 \mathrm{e}-8$ & 20 & 0 & $1.0 \mathrm{e} 5$ & $1.0 \mathrm{e}-8$ \\
\hline
\end{tabular}

Table 6: Initial conditions of 1D gas/liquid Sod shock tube problem.

Figures 7 and 8 compare the numerical solutions from the two schemes with the reference solutions. In figure 7(a), it can be seen that PP-WCNS-IS can capture both the material interface (the left larger density jump) and the shock (the right smaller density jump) with only a few grid points. On the other hand, the first order HLLC scheme is too dissipative that both the material interface and shock are smeared out severely, and hence the two discontinuities cannot be distinguished at this grid resolution. In figures $7(\mathrm{~b})$ and $8(\mathrm{a})$, it can be seen that PP-WCNS-IS can give more accurate solutions in velocity and pressure fields compared to first order HLLC scheme. However, as seen in figure 8(b), PP-WCNS-IS produces a slightly larger undershoot and overshoot at the expansion fan.

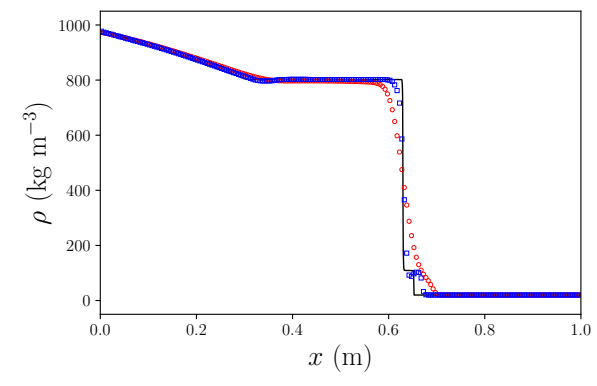

(a) Global density profile

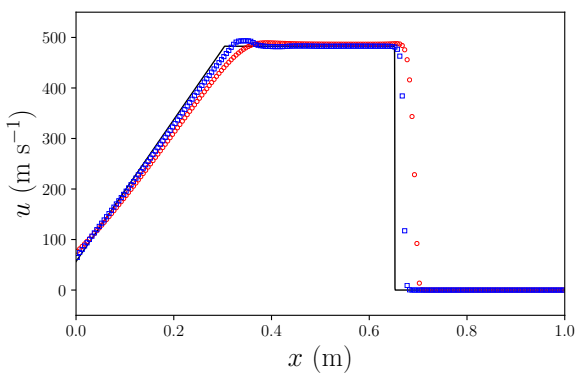

(b) Global velocity profile

Figure 7: Gas/liquid Sod shock tube problem at $t=3 \mathrm{e}-4 \mathrm{~s}$ using different schemes. Black solid line: reference; red circles: HLLC; blue squares: PP-WCNS-IS.

\subsection{One-dimensional planar multi-material Sedov blast wave problem}

This is a multi-material version modified from the well-known single-phase 1D planar Sedov blast wave problem $[37,55,20]$. Initially there is a singularity of highly pressurized air at the center of the domain filled with very low pressure water. Blast waves are created at the original position of the singularity and propagate towards the domain boundaries. The initial conditions are given by table 7. Extrapolations are applied at both boundaries. The spatial domain is $x \in[0,4.0] \mathrm{m}$ and the final time is at $t=1 \mathrm{e}-3 \mathrm{~s}$. Simulations are evolved with constant time steps $\Delta t=2.5 \mathrm{e}-7 \mathrm{~s}$ on a uniform grid with 401 grid points The reference solutions are generated using PP-WCNS-IS with 4001 grid points.

Figures 9 (b) and 10 show the density, velocity, pressure and volume fraction profiles respectively obtained using the two schemes at the final simulation time. It can be seen 


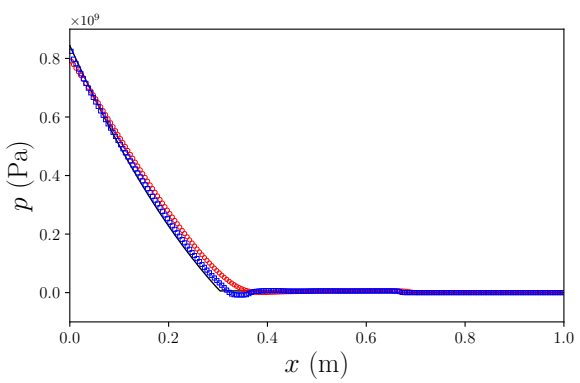

(a) Global pressure profile

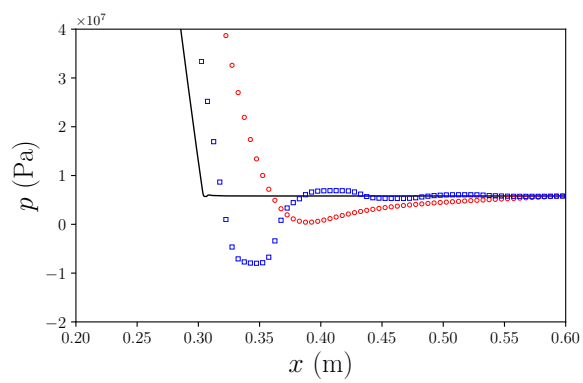

(b) Local pressure profile

Figure 8: Gas/liquid Sod shock tube problem at $t=3 \mathrm{e}-4 \mathrm{~s}$ using different schemes. Black solid line: reference; red circles: HLLC; blue squares: PP-WCNS-IS.

\begin{tabular}{c|ccccc}
\hline & $\begin{array}{c}\alpha_{1} \rho_{1} \\
\left(\mathrm{~kg} \mathrm{~m}^{-3}\right)\end{array}$ & $\begin{array}{c}\alpha_{2} \rho_{2} \\
\left(\mathrm{~kg} \mathrm{~m}^{-3}\right)\end{array}$ & $\begin{array}{c}u \\
\left(\mathrm{~m} \mathrm{~s}^{-1}\right)\end{array}$ & $\begin{array}{c}p \\
(\mathrm{~Pa})\end{array}$ & $\alpha_{1}$ \\
\hline $\begin{array}{c}x<2-0.5 \Delta x \\
\text { or } x>2+0.5 \Delta x \\
\text { otherwise }\end{array}$ & 1000 & $1.0 \mathrm{e}-8$ & 0 & $-3.0 \mathrm{e} 7$ & $1-1.0 \mathrm{e}-8$ \\
\hline
\end{tabular}

Table 7: Initial conditions of 1D planar multi-material Sedov blast wave problem.

that both schemes can capture the blast waves without spurious oscillations. However, the velocity and pressure profiles computed with PP-WCNS-IS are much sharper at the shock fronts while the shocks captured with first order HLLC are severely smeared out due to excess numerical dissipation.

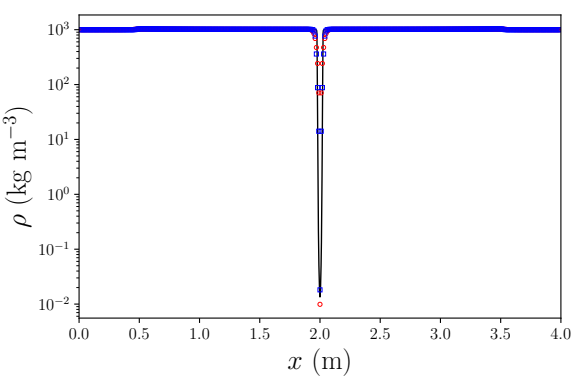

(a) Global density profile

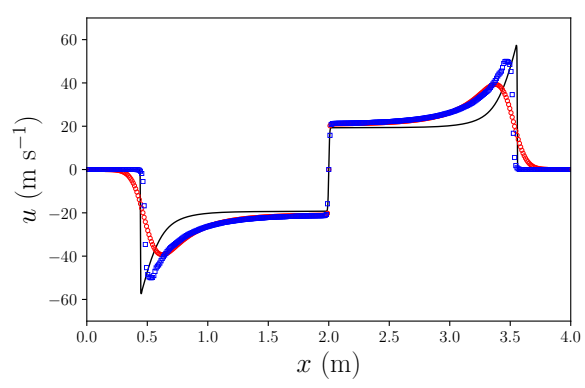

(b) Global velocity profile

Figure 9: 1D multi-material Sedov problem at $t=1 \mathrm{e}-3 \mathrm{~s}$ using different schemes. Black solid line: reference; red circles: HLLC; blue squares: PP-WCNS-IS. 


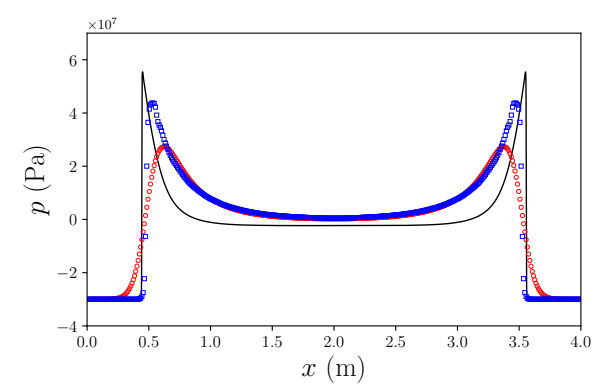

(a) Global pressure profile

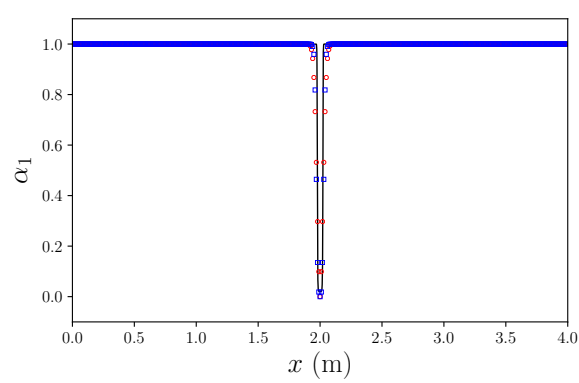

(b) Global water volume fraction profile

Figure 10: 1D multi-material Sedov problem at $t=1 \mathrm{e}-3 \mathrm{~s}$ using different schemes. Black solid line: reference; red circles: HLLC; blue squares: PP-WCNS-IS.

\subsection{Two-dimensional Mach 2.4 shock water cylinder interaction problem by Sembian et al. [38]}

The case of a Mach 2.4 planar shock interacting with a water cylinder in the paper by Sembian et al. [38] is simulated. The purpose of this test case is to investigate the reliability of the flow model with the high-order diffuse interface method for simulating two-phase flows with shocks. Figure 11 shows the schematic of the initial flow field and domain. The water cylinder is initially placed at location $[4 \mathrm{~cm}, 0 \mathrm{~cm}]$. The initial conditions are given by table 8 . Constant extrapolation is used at all domain boundaries. The computations is performed with PP-WCNS-IS on a $3072 \times 2048$ mesh.

\begin{tabular}{c|cccccc}
\hline & $\begin{array}{c}\alpha_{1} \rho_{1} \\
\left(\mathrm{~kg} \mathrm{~m}^{-3}\right)\end{array}$ & $\begin{array}{c}\alpha_{2} \rho_{2} \\
\left(\mathrm{~kg} \mathrm{~m}^{-3}\right)\end{array}$ & $\begin{array}{c}u \\
\left(\mathrm{~m} \mathrm{~s}^{-1}\right)\end{array}$ & $\begin{array}{c}v \\
\left(\mathrm{~m} \mathrm{~s}^{-1}\right)\end{array}$ & $\begin{array}{c}p \\
(\mathrm{~Pa})\end{array}$ & $\alpha_{1}$ \\
\hline pre-shock air & $1.0 \mathrm{e}-8$ & 1.17 & 0 & 0 & $1.01 \mathrm{e} 5$ & $1.0 \mathrm{e}-8$ \\
post-shock air & $1.0 \mathrm{e}-8$ & 3.7579 & 574.57 & 0 & $6.6189 \mathrm{e} 5$ & $1.0 \mathrm{e}-8$ \\
water cylinder & 1000 & $1.0 \mathrm{e}-8$ & 0 & 0 & $1.01 \mathrm{e} 5$ & $1-1.0 \mathrm{e}-8$ \\
\hline
\end{tabular}

Table 8: Initial conditions of 2D Mach 2.4 shock water cylinder interaction problem [38].

The grey schlieren images from the experiment [38] are shown in the left column of figure 12. At the instance when the incident shock interacts with the water column, a shock is reflected upstream since the acoustic impedance of water is higher than that of air. The reflected shock interacts with the incident shock to generate a triple point where the reflected shock, incident shock and a Mach stem along with its slip line conincide. Meanwhile, there is also a shock transmitted into the water column. The transmitted shock travels faster than the shocks outside the water column and it gets reflected as an expansion wave when the transmitted shock reaches the downstream water-air interface. The reflected expansion wave focuses at a point due to the column's downstream concave geometry, where negative presure is produced due to tensile stresses. The reflected expansion wave forms a "horse-shoe" structure after focusing and is reflected 
again at the upstream water-air interface. The expansion wave continues to get reflected inside the water column repeatedly. As the water column is a buff body, the surrounding air separates in the adverse pressure gradient region on the water column surface. Therefore, recirculation regions are created and two counter-rotating vortices are formed downstream of the flow. In the right column of figure 12, the density gradients computed with the simulation results using the high-order PP-WCNS-IS scheme at different times are displayed. Compared with the schlieren images from the experiment, it can be seen that most of the wave features, such as the incident, reflected and transmitted shocks, and expansion waves are captured accurately. Also, the two counter-rotating vortices are reproduced in the simulation.

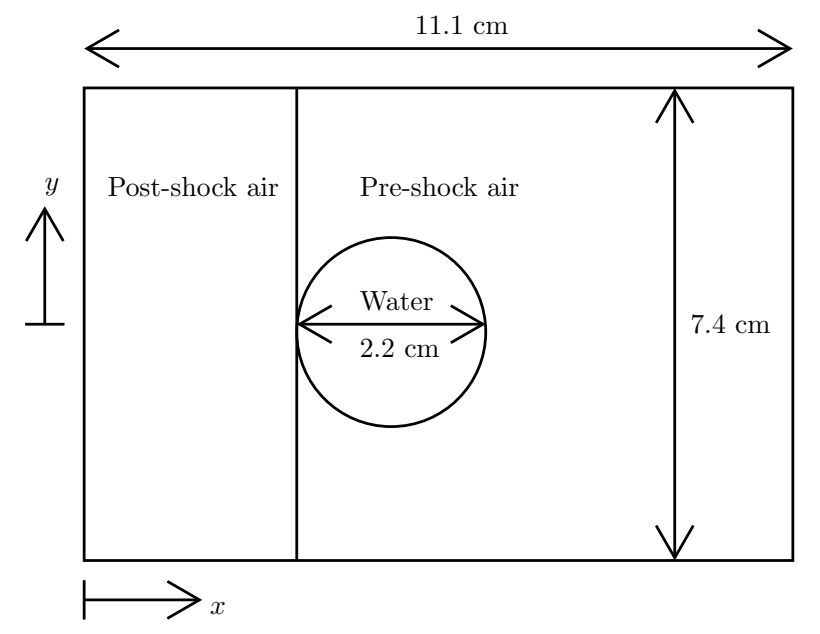

Figure 11: Schematic diagram of 2D Mach 2.4 shock water cylinder interaction problem by Sembian et al. [38].

\subsection{Two-dimensional Mach 10 shock water cylinder interaction problem}

This is the more extreme case of the previous test case. In this problem, a Mach 10 shock wave in air interacts with a water cylinder with diameter of $8 \mathrm{~L}$ in a domain of $[0,30 L] \times[-10 L, 10 L]$, where $L=1 \mathrm{~mm}$ is chosen. Figure 13 shows the schematic of the initial flow field and domain. The water cylinder is initially placed at location $[13 L, 0]$. The initial conditions are given by table 9 . Constant extraploation is used at all domain boundaries. All computations are performed on a $1152 \times 768$ mesh.

This is a very extreme problem due to the high incident shock Mach number initially. The problem is simulated with the first order HLLC and the high-order PP-WCNS-IS. While the first order scheme has no numerical difficulty in this test case, numerical failure is experienced with WCNS-IS without the positivity- and boundedness-preserving limiters as the speed of sound becomes imaginary. This can happen at the strong incident shock, or at the low pressure regions created by the expansion waves inside the water column and at the counter-rotating vortices. The positivity- and boundedness-preserving limiters are necessary for the WCNS-IS scheme in this problem. Figure 14 shows the comparison of numerical schlieren defined as $\exp \left(|\nabla \rho| /|\nabla \rho|_{\max }\right)$ between the two schemes. 


\begin{tabular}{c|cccccc}
\hline & $\begin{array}{c}\alpha_{1} \rho_{1} \\
\left(\mathrm{~kg} \mathrm{~m}^{-3}\right)\end{array}$ & $\begin{array}{c}\alpha_{2} \rho_{2} \\
\left(\mathrm{~kg} \mathrm{~m}^{-3}\right)\end{array}$ & $\begin{array}{c}u \\
\left(\mathrm{~m} \mathrm{~s}^{-1}\right)\end{array}$ & $\begin{array}{c}v \\
\left(\mathrm{~m} \mathrm{~s}^{-1}\right)\end{array}$ & $\begin{array}{c}p \\
(\mathrm{~Pa})\end{array}$ & $\alpha_{1}$ \\
\hline pre-shock air & $1.0 \mathrm{e}-8$ & 1.2 & 0 & 0 & $1.0 \mathrm{e} 5$ & $1.0 \mathrm{e}-8$ \\
post-shock air & $1.0 \mathrm{e}-8$ & 6.8571 & $2.8179 \mathrm{e} 3$ & 0 & $1.165 \mathrm{e} 7$ & $1.0 \mathrm{e}-8$ \\
water cylinder & 1000 & $1.0 \mathrm{e}-8$ & 0 & 0 & $1.0 \mathrm{e} 5$ & $1-1.0 \mathrm{e}-8$ \\
\hline
\end{tabular}

Table 9: Initial conditions of 2D Mach 10 shock water cylinder interaction problem.

From the figure, we can see that the PP-WCNS-IS has much thinner interface thickness over time compared with first order HLLC scheme and this is consistent with other test problems. Vortical features produced by the hydrodynamic instability due to baroclinic torque are observed at the interface for PP-WCNS-IS as time evolves. However, the first order scheme is too dissipative to produce the roll-up of the interfaces at the chosen grid resolution. The comparison of speed of sound between the two schemes can be seen in figure 15. Finally, the volume fraction fields of both schemes are shown in figure 16. It should be noted that the volume fraction field is also verified to be always bounded by the corresponding threshold chosen in the positivity- and boundedness-preserving limiters for PP-WCNS-IS.

\subsection{Two-dimensional Mach 100 water jet problem}

This test case is a multi-phase version of the popular Mach 2000 jet problem first proposed by Zhang and Shu [53]. In this problem, a Mach 100 water jet enters a domain full of ambient air. The domain size is $[0, L] \times[-0.25 L, 0.25 L]$, where $L=1 \mathrm{~m}$ is chosen. The initial conditions of the ambient air are given by table 10. Constant extrapolation is used at top, bottom and right boundaries. The left boundary is described by Dirichlet boundary conditions given by table 11 . The speed of the jet is $1.5 \mathrm{e} 5 \mathrm{~m} \mathrm{~s}^{-1}$, which is around Mach 100 with respect to the sound speed in the water jet. The computations are performed on a $1024 \times 512$ mesh.

\begin{tabular}{cccccc}
\hline $\begin{array}{c}\alpha_{1} \rho_{1} \\
\left(\mathrm{~kg} \mathrm{~m}^{-3}\right)\end{array}$ & $\begin{array}{c}\alpha_{2} \rho_{2} \\
\left(\mathrm{~kg} \mathrm{~m}^{-3}\right)\end{array}$ & $\begin{array}{c}u \\
\left(\mathrm{~m} \mathrm{~s}^{-1}\right)\end{array}$ & $\begin{array}{c}v \\
\left(\mathrm{~m} \mathrm{~s}^{-1}\right)\end{array}$ & $\begin{array}{c}p \\
(\mathrm{~Pa})\end{array}$ & $\alpha_{1}$ \\
\hline $1.0 \mathrm{e}-8$ & 1 & 0 & 0 & $1.0 \mathrm{e} 5$ & $1.0 \mathrm{e}-8$ \\
\hline
\end{tabular}

Table 10: Initial conditions of 2D Mach 100 water jet problem.

The comparison of speed of sound between the two schemes is shown in figure 17 . Despite the large jump in sound speed across the bow shock ahead of the high speed water jet, none of the schemes fail due to the positivity-preserving properties of both schemes for sound speed. The main difference between the two schemes is at the water jet front where the interface at the water jet front produced by first order HLLC scheme is heavily smeared out while that of PP-WCNS-IS is reasonably captured with only a few grid points. There are also some small but obvious numerical artificts at the bow shock in the solutions computed with the first order scheme. In figure 18, the numerical schilren 


\begin{tabular}{c|cccccc}
\hline & $\begin{array}{c}\alpha_{1} \rho_{1} \\
\left(\mathrm{~kg} \mathrm{~m}^{-3}\right)\end{array}$ & $\begin{array}{c}\alpha_{2} \rho_{2} \\
\left(\mathrm{~kg} \mathrm{~m}^{-3}\right)\end{array}$ & $\begin{array}{c}u \\
\left(\mathrm{~m} \mathrm{~s}^{-1}\right)\end{array}$ & $\begin{array}{c}v \\
\left(\mathrm{~m} \mathrm{~s}^{-1}\right)\end{array}$ & $\begin{array}{c}p \\
(\mathrm{~Pa})\end{array}$ & $\alpha_{1}$ \\
\hline $\begin{array}{c}y \in[-0.05 L, 0.05 L] \\
\text { otherwise }\end{array}$ & 1000 & $1.0 \mathrm{e}-8$ & $1.5 \mathrm{e} 5$ & 0 & $1.0 \mathrm{e} 5$ & $1-1.0 \mathrm{e}-8$ \\
\hline
\end{tabular}

Table 11: Left boundary conditions of 2D Mach 100 water jet problem.

between the two schemes are compared. Since the interface water jet front is seriously diffused, the shape of the water-air interface at the water front cannot be visualized at all. Figure 19 compares the volume fraction field of water at different times. The volume fraction field is verified to be bounded in PP-WCNS-IS. Similar to other fields, volume fraction interface at the water jet front is very diffused for the first order scheme compared to the high-order PP-WCNS-IS.

\section{Concluding remarks}

In this work, limiting procedures were proposed on a high-order finite difference scheme that can preserve the positivity of partial density of each phase and squared speed of sound and also the boundedness of the volume fractions in 1D and multi-dimensional gas-liquid two-phase problems under a mild assumption on the material properties of the gas and liquid. The procedures consist of two stages which limit the WENO interpolation and flux reconstruction respectively in the high-order WCNS-IS algorithm. Discrete conservation of solutions for the conservation equations in the five-equation model is still maintained even with the limiting. The overall positivity- and boundedness-preserving scheme, PP-WCNS-IS, was tested with different severe problems, under suitable CFL conditions. Comparison between the results of the first order HLLC scheme and PPWCNS-IS showed the low dissipation and high resolution properties of the latter scheme while its robustness is also ensured. The positivity- and boundedness-preserving limiting procedures can also be potentially used with any conservative finite difference and finite volume schemes for gas-liquid two-phase flows. Future work includes generalization of the positivity- and boundedness-preserving limiters to more general equation of states and multi-phase flows with more than two species. There will also be future investigations on the use of the diffuse interface method for simulations of space vehicle launches with water-based sound suppression systems $[47,26]$.

\section{Acknowledgments}

This work was partially supported by the NASA Exploration Ground Systems (EGS) program and the NASA Engineering and Safety Center (NESC). Computer time has been provided by the NASA Advanced Supercomputing (NAS) facility at NASA Ames Research Center. We also gratefully acknowledge Dr. Bruce T. Vu, Dr. Jeffrey A. Housman and Dr. Oliver M. Browne for valuable discussions. 


\section{Appendix A. Characteristic decomposition}

The choice of variables for WENO reconstruction and interpolation is very critical to avoid spurious oscillations across discontinuities, especially across the material interfaces. It was shown in [23] that if conservative variables are chosen for WENO reconstruction, spurious oscillations will appear at material interfaces. Primitive variables were suggested $[23,8,49]$ for reconstruction and interpolation in order to maintain pressure and velocity equilibria across interfaces. Furthermore, WENO reconstruction and interpolation of characteristic variables projected from primitive variables can avoid the interaction of discontinuities in different characteristic fields. To illustrate how the primitive variables are converted into characteristic variables, we follow previous works $[8,49]$ by first rewriting the $2 \mathrm{D}$ governing equations in the quasi-linear primitive form:

$$
\frac{\partial \mathbf{V}}{\partial t}+\mathbf{A}(\mathbf{V}) \frac{\partial \mathbf{V}}{\partial x}+\mathbf{B}(\mathbf{V}) \frac{\partial \mathbf{V}}{\partial y}=0,
$$

where $\mathbf{V}$ is the vector of primitive variables. $\mathbf{V}$ and matrix $\mathbf{A}$ are given by:

$$
\begin{aligned}
\mathbf{V} & =\left(\begin{array}{c}
\alpha_{1} \rho_{1} \\
\alpha_{2} \rho_{2} \\
u \\
v \\
p \\
\alpha_{1}
\end{array}\right), \quad \mathbf{A}=\left(\begin{array}{cccccc}
u & 0 & \alpha_{1} \rho_{1} & 0 & 0 & 0 \\
0 & u & \alpha_{2} \rho_{2} & 0 & 0 & 0 \\
0 & 0 & u & 0 & \frac{1}{\rho} & 0 \\
0 & 0 & 0 & u & 0 & 0 \\
0 & 0 & \rho c^{2} & 0 & u & 0 \\
0 & 0 & 0 & 0 & 0 & u
\end{array}\right), \\
\mathbf{B} & =\left(\begin{array}{cccccc}
v & 0 & 0 & \alpha_{1} \rho_{1} & 0 & 0 \\
0 & v & 0 & \alpha_{2} \rho_{2} & 0 & 0 \\
0 & 0 & v & 0 & 0 & 0 \\
0 & 0 & 0 & v & \frac{1}{\rho} & 0 \\
0 & 0 & 0 & \rho c^{2} & v & 0 \\
0 & 0 & 0 & 0 & 0 & v
\end{array}\right) .
\end{aligned}
$$

The eigenvectors of the matrices $\mathbf{A}$ and $\mathbf{B}$ have to be determined first in order to transform primitive variables to characteristic variables. The eigenvalue decompositions of the matrices are given by:

$$
\mathbf{A}=\mathbf{R}_{\mathbf{A}} \boldsymbol{\Lambda}_{\mathbf{A}} \mathbf{R}_{\mathrm{A}}^{-1}, \quad \mathbf{B}=\mathbf{R}_{\mathbf{B}} \boldsymbol{\Lambda}_{\mathbf{B}} \mathbf{R}_{\mathbf{B}}^{-1},
$$


where $\mathbf{R}_{\mathbf{A}}, \mathbf{R}_{\mathbf{A}}^{-1}$ and $\boldsymbol{\Lambda}_{\mathbf{A}}$ are given by:

$$
\begin{aligned}
\mathbf{R}_{\mathbf{A}} & =\left(\begin{array}{cccccc}
-\frac{\alpha_{1} \rho_{1}}{2 c} & 1 & 0 & 0 & 0 & \frac{\alpha_{1} \rho_{1}}{2 c} \\
-\frac{\alpha_{2} \rho_{2}}{2 c} & 0 & 1 & 0 & 0 & \frac{\alpha_{2} \rho_{2}}{2 c} \\
\frac{1}{2} & 0 & 0 & 0 & 0 & \frac{1}{2} \\
0 & 0 & 0 & 1 & 0 & 0 \\
-\frac{\rho c}{2} & 0 & 0 & 0 & 0 & \frac{\rho c}{2} \\
0 & 0 & 0 & 0 & 1 & 0
\end{array}\right), \quad \mathbf{R}_{\mathbf{A}}^{-1}=\left(\begin{array}{cccccc}
0 & 0 & 1 & 0 & -\frac{1}{\rho c} & 0 \\
1 & 0 & 0 & 0 & -\frac{\alpha_{1} \rho_{1}}{\rho c^{2}} & 0 \\
0 & 1 & 0 & 0 & -\frac{\alpha_{2} \rho_{2}}{\rho c^{2}} & 0 \\
0 & 0 & 0 & 1 & 0 & 0 \\
0 & 0 & 0 & 0 & 0 & 1 \\
0 & 0 & 1 & 0 & \frac{1}{\rho c} & 0
\end{array}\right), \\
\boldsymbol{\Lambda}_{\mathbf{A}} & =\left(\begin{array}{cccccc}
u-c & 0 & 0 & 0 & 0 & 0 \\
0 & u & 0 & 0 & 0 & 0 \\
0 & 0 & u & 0 & 0 & 0 \\
0 & 0 & 0 & u & 0 & 0 \\
0 & 0 & 0 & 0 & u & 0 \\
0 & 0 & 0 & 0 & 0 & u+c
\end{array}\right) .
\end{aligned}
$$

$\mathbf{R}_{\mathrm{B}}, \mathbf{R}_{\mathrm{B}}^{-1}$ and $\boldsymbol{\Lambda}_{\mathrm{B}}$ have similar corresponding forms.

\section{Appendix B. Convergence analysis of the incremental-stencil WCNS}

The convergence analysis discussed in this section is the extension of the convergence analysis of Yan et al. [50] to HCS with WENO-IS interpolation. Assume that we have a 1D scalar hyperbolic conservation law of dependent variable $u$ :

$$
\partial_{t} u+\partial_{x} F=0,
$$

where $F=F(u)$ is the flux. Under the assumption that $\partial F(u) / \partial u>0$ with perfect upwinding scheme, $\tilde{F}_{i+\frac{1}{2}}=F\left(\tilde{u}_{i+\frac{1}{2}, L}\right)$, where $\tilde{u}_{i+\frac{1}{2}, L}$ is the left-biased WENO interpolated value of $u$. The subscript " $L$ " is dropped in this section for convenience.

After Taylor series expansion of the interpolation equations (84)-(87) for the substencils, we obtain:

$$
\tilde{u}_{i+\frac{1}{2}}^{k}= \begin{cases}u_{i+\frac{1}{2}}+A_{i}^{k} \Delta x^{2}+\mathcal{O}\left(\Delta x^{3}\right), & \text { if } k=0,1, \\ u_{i+\frac{1}{2}}+A_{i}^{k} \Delta x^{3}+\mathcal{O}\left(\Delta x^{4}\right), & \text { if } k=2,3\end{cases}
$$

where $A_{i}^{0}=u_{i}^{\prime \prime} / 8, A_{i}^{1}=-3 u_{i}^{\prime \prime} / 8, A_{i}^{2}=-u_{i}^{\prime \prime \prime} / 16$, and $A_{i}^{3}=-5 u_{i}^{\prime \prime \prime} / 16$. If we replace the nonlinear weights with the corresponding linear weights in equation (91),

$$
\sum_{k=0}^{3} d_{k} \tilde{u}_{i+\frac{1}{2}}^{k}=u_{i+\frac{1}{2}}+B_{i} \Delta x^{5}+\mathcal{O}\left(\Delta x^{6}\right)
$$

where $B_{i}=-3 u_{i}^{(5)} / 256$.

From equation (91),

$$
\begin{aligned}
\tilde{u}_{i+\frac{1}{2}} & =\sum_{k=0}^{3} \omega_{k} \tilde{u}_{i+\frac{1}{2}}^{(k)} \\
& =\sum_{k=0}^{3} d_{k} \tilde{u}_{i+\frac{1}{2}}^{(k)}+\sum_{k=0}^{3}\left(\omega_{k}-d_{k}\right) \tilde{u}_{i+\frac{1}{2}}^{(k)} .
\end{aligned}
$$


Using equations (B.2) and (B.3), the equation above becomes:

$$
\begin{aligned}
\tilde{u}_{i+\frac{1}{2}}= & u_{i+\frac{1}{2}}+B_{i} \Delta x^{5}+\mathcal{O}\left(\Delta x^{6}\right) \\
& +\sum_{k=0}^{1}\left\{\left(\omega_{k}-d_{k}\right)\left[u_{i+\frac{1}{2}}+A_{i}^{k} \Delta x^{2}+\mathcal{O}\left(\Delta x^{3}\right)\right]\right\} \\
& +\sum_{k=2}^{3}\left\{\left(\omega_{k}-d_{k}\right)\left[u_{i+\frac{1}{2}}+A_{i}^{k} \Delta x^{3}+\mathcal{O}\left(\Delta x^{4}\right)\right]\right\} \\
= & u_{i+\frac{1}{2}}+B_{i} \Delta x^{5}+\mathcal{O}\left(\Delta x^{6}\right)+u_{i+\frac{1}{2}} \sum_{k=0}^{3}\left(\omega_{k}-d_{k}\right) \\
& +\Delta x^{2} \sum_{k=0}^{1}\left[A_{i}^{k}\left(\omega_{k}-d_{k}\right)\right]+\Delta x^{3} \sum_{k=2}^{3}\left[A_{i}^{k}\left(\omega_{k}-d_{k}\right)\right] \\
& +\sum_{k=0}^{1}\left[\left(\omega_{k}-d_{k}\right) \mathcal{O}\left(\Delta x^{3}\right)\right]+\sum_{k=2}^{3}\left[\left(\omega_{k}-d_{k}\right) \mathcal{O}\left(\Delta x^{4}\right)\right]
\end{aligned}
$$

The derivation is similar for $\tilde{u}_{i-\frac{1}{2}}$. Therefore,

$$
\begin{aligned}
\tilde{u}_{i \pm \frac{1}{2}}-u_{i \pm \frac{1}{2}}= & B_{i} \Delta x^{5}+u_{i \pm \frac{1}{2}} \sum_{k=0}^{3}\left(\omega_{k}^{ \pm}-d_{k}\right)+\Delta x^{2} \sum_{k=0}^{1}\left[A_{i}^{k}\left(\omega_{k}^{ \pm}-d_{k}\right)\right] \\
& +\Delta x^{3} \sum_{k=2}^{3}\left[A_{i}^{k}\left(\omega_{k}^{ \pm}-d_{k}\right)\right]+\sum_{k=0}^{1}\left[\left(\omega_{k}^{ \pm}-d_{k}\right) \mathcal{O}\left(\Delta x^{3}\right)\right] \\
& +\sum_{k=2}^{3}\left[\left(\omega_{k}^{ \pm}-d_{k}\right) \mathcal{O}\left(\Delta x^{4}\right)\right]+\mathcal{O}\left(\Delta x^{6}\right)
\end{aligned}
$$

at midpoints $i+1 / 2$ and $i-1 / 2$. The superscript \pm is added to $\omega_{k}$ to distinguish the values at the two different midpoints. Note that no \pm is added to $d_{k}, A_{i}^{k}$ and $B_{i}$ since they have the same values at the two midpoints. Using the fact that $\sum_{k=0}^{3} \omega_{k}^{ \pm}=\sum_{k=0}^{3} d_{k}=1$, we finally get:

$$
\begin{aligned}
\tilde{u}_{i \pm \frac{1}{2}}-u_{i \pm \frac{1}{2}}= & B_{i} \Delta x^{5}+\Delta x^{2} \sum_{k=0}^{1}\left[A_{i}^{k}\left(\omega_{k}^{ \pm}-d_{k}\right)\right]+\Delta x^{3} \sum_{k=2}^{3}\left[A_{i}^{k}\left(\omega_{k}^{ \pm}-d_{k}\right)\right] \\
& +\sum_{k=0}^{1}\left[\left(\omega_{k}^{ \pm}-d_{k}\right) \mathcal{O}\left(\Delta x^{3}\right)\right]+\sum_{k=2}^{3}\left[\left(\omega_{k}^{ \pm}-d_{k}\right) \mathcal{O}\left(\Delta x^{4}\right)\right] \\
& +\mathcal{O}\left(\Delta x^{6}\right) .
\end{aligned}
$$

We will show that the WCNS-IS is fifth order accurate if $\omega_{k}^{ \pm}-d_{k}=\mathcal{O}\left(\Delta x^{4}\right)$. If we 
assume that $\omega_{k}^{ \pm}-d_{k}=\mathcal{O}\left(\Delta x^{4}\right)$, by Taylor series expansion,

$$
\begin{aligned}
\tilde{F}_{i \pm \frac{1}{2}} & =F_{i \pm \frac{1}{2}}+\left.\frac{\partial F}{\partial u}\right|_{i \pm \frac{1}{2}}\left(\tilde{u}_{i \pm \frac{1}{2}}-u_{i \pm \frac{1}{2}}\right)+\left.\frac{1}{2} \frac{\partial^{2} F}{\partial u^{2}}\right|_{i \pm \frac{1}{2}}\left(\tilde{u}_{i \pm \frac{1}{2}}-u_{i \pm \frac{1}{2}}\right)^{2}+\cdots \\
& =F_{i \pm \frac{1}{2}}+\left.\frac{\partial F}{\partial u}\right|_{i \pm \frac{1}{2}}\left(\tilde{u}_{i \pm \frac{1}{2}}-u_{i \pm \frac{1}{2}}\right)+\mathcal{O}\left(\Delta x^{10}\right) .
\end{aligned}
$$

Substituting equations (B.7) and (B.8) into the 1D version of equation (77) (noticing $F$ is the scalar version of $\mathbf{G}^{x}$ here) and using Taylor-sereis expanded equation (78) (assuming $\psi=256 / 175)$,

$$
\begin{aligned}
\left.\widehat{\partial F}\right|_{i}= & \left.\frac{\partial F}{\partial x}\right|_{i}+\mathcal{O}\left(\Delta x^{8}\right) \\
+ & \psi\left\{\left.\frac{\partial F}{\partial u}\right|_{i+\frac{1}{2}} \sum_{k=0}^{1}\left[\left(\omega_{k}^{+}-d_{k}\right) \mathcal{O}\left(\Delta x^{2}\right)\right]\right. \\
& \left.-\left.\frac{\partial F}{\partial u}\right|_{i-\frac{1}{2}} \sum_{k=0}^{1}\left[\left(\omega_{k}^{-}-d_{k}\right) \mathcal{O}\left(\Delta x^{2}\right)\right]\right\} \\
+ & \psi\left\{\left.\frac{\partial F}{\partial u}\right|_{i+\frac{1}{2}} \sum_{k=2}^{3}\left[\left(\omega_{k}^{+}-d_{k}\right) \mathcal{O}\left(\Delta x^{3}\right)\right]\right. \\
& \left.-\left.\frac{\partial F}{\partial u}\right|_{i-\frac{1}{2}} \sum_{k=2}^{3}\left[\left(\omega_{k}^{-}-d_{k}\right) \mathcal{O}\left(\Delta x^{3}\right)\right]\right\} \\
& +\psi \Delta x^{4} B_{i}\left(\left.\frac{\partial F}{\partial u}\right|_{i+\frac{1}{2}}-\left.\frac{\partial F}{\partial u}\right|_{i-\frac{1}{2}}\right) \\
& +\psi \Delta x\left\{\left.\frac{\partial F}{\partial u}\right|_{i+\frac{1}{2}} \sum_{k=0}^{1}\left[A_{i}^{k}\left(\omega_{k}^{+}-d_{k}\right)\right]-\left.\frac{\partial F}{\partial u}\right|_{i-\frac{1}{2}} \sum_{k=0}^{1}\left[A_{i}^{k}\left(\omega_{k}^{-}-d_{k}\right)\right]\right\} \\
& +\psi \Delta x^{2}\left\{\left.\frac{\partial F}{\partial u}\right|_{i+\frac{1}{2}} \sum_{k=2}^{3}\left[A_{i}^{k}\left(\omega_{k}^{+}-d_{k}\right)\right]-\left.\frac{\partial F}{\partial u}\right|_{i-\frac{1}{2}} \sum_{k=2}^{3}\left[A_{i}^{k}\left(\omega_{k}^{-}-d_{k}\right)\right]\right\} \\
& +\mathcal{O}\left(\Delta x^{5}\right) .
\end{aligned}
$$

Also, by Taylor series expansion,

$$
\begin{aligned}
\left.\frac{\partial F}{\partial u}\right|_{i+\frac{1}{2}} & =\left.\frac{\partial F}{\partial u}\right|_{i}+\left.\frac{\partial^{2} F}{\partial u^{2}}\right|_{i}\left(u_{i+\frac{1}{2}}-u_{i}\right)+\left.\frac{1}{2} \frac{\partial^{3} F}{\partial u^{3}}\right|_{i}\left(u_{i+\frac{1}{2}}-u_{i}\right)^{2}+\cdots, \\
u_{i+\frac{1}{2}} & =u_{i}+\left.\frac{\Delta x}{2} \frac{\partial u}{\partial x}\right|_{i}+\left.\frac{\Delta x^{2}}{8} \frac{\partial^{2} u}{\partial x^{2}}\right|_{i}+\mathcal{O}\left(\Delta x^{3}\right) .
\end{aligned}
$$


Therefore,

$$
\begin{aligned}
\left.\frac{\partial F}{\partial u}\right|_{i+\frac{1}{2}}= & \left.\frac{\partial F}{\partial u}\right|_{i}+\left.\frac{\partial^{2} F}{\partial u^{2}}\right|_{i}\left[\left.\frac{\Delta x}{2} \frac{\partial u}{\partial x}\right|_{i}+\left.\frac{\Delta x^{2}}{8} \frac{\partial^{2} u}{\partial x^{2}}\right|_{i}+\mathcal{O}\left(\Delta x^{3}\right)\right] \\
& +\left.\frac{1}{2} \frac{\partial^{3} F}{\partial u^{3}}\right|_{i}\left[\left.\frac{\Delta x}{2} \frac{\partial u}{\partial x}\right|_{i}+\mathcal{O}\left(\Delta x^{2}\right)\right]^{2}+\mathcal{O}\left(\Delta x^{3}\right) \\
= & \left.\frac{\partial F}{\partial u}\right|_{i}+\frac{\Delta x}{2} \frac{\partial^{2} F}{\partial u^{2}}\left|\frac{\partial u}{\partial x}\right|_{i}+\left.\left.\frac{\Delta x^{2}}{8} \frac{\partial^{2} F}{\partial u^{2}}\right|_{i} \frac{\partial^{2} u}{\partial x^{2}}\right|_{i}+\left.\frac{\Delta x^{2}}{8} \frac{\partial^{3} F}{\partial u^{3}}\right|_{i}\left(\left.\frac{\partial u}{\partial x}\right|_{i}\right)^{2} \\
& +\mathcal{O}\left(\Delta x^{3}\right) .
\end{aligned}
$$

Similarly, by Taylor series expansion,

$$
\begin{aligned}
\left.\frac{\partial F}{\partial u}\right|_{i-\frac{1}{2}}= & \left.\frac{\partial F}{\partial u}\right|_{i}+\left.\frac{\partial^{2} F}{\partial u^{2}}\right|_{i}\left[-\left.\frac{\Delta x}{2} \frac{\partial u}{\partial x}\right|_{i}+\left.\frac{\Delta x^{2}}{8} \frac{\partial^{2} u}{\partial x^{2}}\right|_{i}+\mathcal{O}\left(\Delta x^{3}\right)\right] \\
& +\left.\frac{1}{2} \frac{\partial^{3} F}{\partial u^{3}}\right|_{i}\left[-\left.\frac{\Delta x}{2} \frac{\partial u}{\partial x}\right|_{i}+\mathcal{O}\left(\Delta x^{2}\right)\right]^{2}+\mathcal{O}\left(\Delta x^{3}\right) \\
= & \left.\frac{\partial F}{\partial u}\right|_{i}-\frac{\Delta x}{2} \frac{\partial^{2} F}{\partial u^{2}}\left|\frac{\partial u}{\partial x}\right|_{i}+\left.\left.\frac{\Delta x^{2}}{8} \frac{\partial^{2} F}{\partial u^{2}}\right|_{i} \frac{\partial^{2} u}{\partial x^{2}}\right|_{i}+\left.\frac{\Delta x^{2}}{8} \frac{\partial^{3} F}{\partial u^{3}}\right|_{i}\left(\left.\frac{\partial u}{\partial x}\right|_{i}\right)^{2} \\
& +\mathcal{O}\left(\Delta x^{3}\right) .
\end{aligned}
$$


As a result, equation (B.9) is simplified to:

$$
\begin{aligned}
\left.\widehat{\partial F}\right|_{i}= & \left.\frac{\partial F}{\partial x}\right|_{i} \\
+ & \left\{\left.\frac{\partial F}{\partial u}\right|_{i+\frac{1}{2}} \sum_{k=0}^{1}\left[\left(\omega_{k}^{+}-d_{k}\right) \mathcal{O}\left(\Delta x^{2}\right)\right]\right. \\
& \left.-\left.\frac{\partial F}{\partial u}\right|_{i-\frac{1}{2}} \sum_{k=0}^{1}\left[\left(\omega_{k}^{-}-d_{k}\right) \mathcal{O}\left(\Delta x^{2}\right)\right]\right\} \\
+ & \left\{\left.\frac{\partial F}{\partial u}\right|_{i+\frac{1}{2}} \sum_{k=2}^{3}\left[\left(\omega_{k}^{+}-d_{k}\right) \mathcal{O}\left(\Delta x^{3}\right)\right]\right. \\
& \left.-\left.\frac{\partial F}{\partial u}\right|_{i-\frac{1}{2}} \sum_{k=2}^{3}\left[\left(\omega_{k}^{-}-d_{k}\right) \mathcal{O}\left(\Delta x^{3}\right)\right]\right\} \\
+ & {\left[\left.\left.\left.\frac{\psi \Delta x^{5} B_{i}}{\partial x^{2}} \frac{\partial^{2} F}{\partial u^{2}}\right|_{i} \frac{\partial u}{\partial x}\right|_{i}\right|_{i}+\left[\left.\psi u_{i} \frac{\partial u}{\partial x}\right|_{i}+\mathcal{O}\left(\Delta x^{4}\right)\right]+\mathcal{O}\left(\Delta x^{3}\right)\right] \sum_{k=0}^{1}\left[A_{i}^{k}\left(\omega_{k}^{+}-\omega_{k}^{-}\right)\right] } \\
& \left\{\sum_{k=0}^{1}\left[A_{i}^{k}\left(\omega_{k}^{+}-d_{k}\right)\right]+\sum_{k=0}^{1}\left[A_{i}^{k}\left(\omega_{k}^{-}-d_{k}\right)\right]\right\} \\
+ & {\left[\left.\psi \Delta x^{2} \frac{\partial F}{\partial u}\right|_{i}+\mathcal{O}\left(\Delta x^{4}\right)\right] \sum_{k=2}^{3}\left[A_{i}^{k}\left(\omega_{k}^{+}-\omega_{k}^{-}\right)\right] } \\
+ & {\left[\left.\left.\frac{\psi \Delta x^{3}}{2} \frac{\partial^{2} F}{\partial u^{2}}\right|_{i} \frac{\partial u}{\partial x}\right|_{i}+\mathcal{O}\left(\Delta x^{5}\right)\right] } \\
& \left\{\sum_{k=2}^{3}\left[A_{i}^{k}\left(\omega_{k}^{+}-d_{k}\right)\right]+\sum_{k=2}^{3}\left[A_{i}^{k}\left(\omega_{k}^{-}-d_{k}\right)\right]\right\} \\
+ & \mathcal{O}\left(\Delta x^{5}\right) .
\end{aligned}
$$

It can be seen from equation (B.14) that a sufficient condition for fifth order convergence is $\omega_{k}^{ \pm}-d_{k}=\mathcal{O}\left(\Delta x^{4}\right)$, which is already assumed earlier.

To prove that $\omega_{k}^{ \pm}-d_{k}=\mathcal{O}\left(\Delta x^{4}\right)$ is true, we can perform Taylor series expansion on 
the smoothness indicators given by equations (95)-(99),

$$
\begin{aligned}
\beta_{0}= & \left(u_{i}^{\prime}\right)^{2} \Delta x^{2}+u_{i}^{\prime} u_{i}^{\prime \prime} \Delta x^{3}+\left(\frac{u_{i}^{\prime} u_{i}^{\prime \prime \prime}}{3}+\frac{\left(u_{i}^{\prime \prime}\right)^{2}}{4}\right) \Delta x^{4}+\left(\frac{u_{i}^{\prime} u_{i}^{(4)}}{12}+\frac{u_{i}^{\prime \prime} u_{i}^{\prime \prime \prime}}{6}\right) \Delta x^{5} \\
& +\mathcal{O}\left(\Delta x^{6}\right), \\
\beta_{1}= & \left(u_{i}^{\prime}\right)^{2} \Delta x^{2}-u_{i}^{\prime} u_{i}^{\prime \prime} \Delta x^{3}+\left(\frac{u_{i}^{\prime} u_{i}^{\prime \prime \prime}}{3}+\frac{\left(u_{i}^{\prime \prime}\right)^{2}}{4}\right) \Delta x^{4} \\
& +\left(-\frac{u_{i}^{\prime} u_{i}^{(4)}}{12}-\frac{u_{i}^{\prime \prime} u_{i}^{\prime \prime \prime}}{6}\right) \Delta x^{5}+\mathcal{O}\left(\Delta x^{6}\right), \\
\beta_{01}= & \left(u_{i}^{\prime}\right)^{2} \Delta x^{2}+\left(\frac{u_{i}^{\prime} u_{i}^{\prime \prime \prime}}{3}+\frac{13\left(u_{i}^{\prime \prime}\right)^{2}}{12}\right) \Delta x^{4}+\mathcal{O}\left(\Delta x^{6}\right), \\
\beta_{2}= & \left(u_{i}^{\prime}\right)^{2} \Delta x^{2}+\left(-\frac{2 u_{i}^{\prime} u_{i}^{\prime \prime \prime}}{3}+\frac{13\left(u_{i}^{\prime \prime}\right)^{2}}{12}\right) \Delta x^{4}+\left(-\frac{u_{i}^{\prime} u_{i}^{(4)}}{2}+\frac{13 u_{i}^{\prime \prime} u_{i}^{\prime \prime \prime}}{6}\right) \Delta x^{5} \\
& +\mathcal{O}\left(\Delta x^{6}\right), \\
\beta_{3}= & \left(u_{i}^{\prime}\right)^{2} \Delta x^{2}+\left(-\frac{2 u_{i}^{\prime} u_{i}^{\prime \prime \prime}}{3}+\frac{13\left(u_{i}^{\prime \prime}\right)^{2}}{12}\right) \Delta x^{4}+\left(\frac{u_{i}^{\prime} u_{i}^{(4)}}{2}-\frac{13 u_{i}^{\prime \prime} u_{i}^{\prime \prime \prime}}{6}\right) \Delta x^{5} \\
& +\mathcal{O}\left(\Delta x^{6}\right) .
\end{aligned}
$$

The Taylor series expansion of reference smoothness indicator (equation (101)) gives:

$$
\tau_{5}=\left(u_{i}^{\prime \prime \prime}\right)^{2} \Delta x^{6}+\mathcal{O}\left(\Delta x^{8}\right) .
$$

Therefore,

$$
\frac{\tau_{5}}{\beta_{k}+\epsilon}=\mathcal{O}\left(\Delta x^{4}\right)
$$

provided not at critical points. As explained in Borges et al. [4] and Wang et al. [48], this is sufficient to have $\omega_{k}^{ \pm}-d_{k}=\mathcal{O}\left(\Delta x^{4}\right)$.

\section{References}

[1] G. Allaire, S. Clerc, and S. Kokh. A five-equation model for the simulation of interfaces between compressible fluids. Journal of Computational Physics, 181(2):577-616, 2002.

[2] M. Aslani and J. D. Regele. A localized artificial diffusivity method to simulate compressible multiphase flows using the stiffened gas equation of state. International Journal for Numerical Methods in Fluids, 88(9):413-433, 2018.

[3] P. Batten, N. Clarke, C. Lambert, and D. Causon. On the choice of wavespeeds for the HLLC Riemann solver. SIAM Journal on Scientific Computing, 18(6):1553-1570, 1997.

[4] R. Borges, M. Carmona, B. Costa, and W. S. Don. An improved weighted essentially non-oscillatory scheme for hyperbolic conservation laws. Journal of Computational Physics, 227(6):3191-3211, 2008.

[5] C.-H. Chang and M.-S. Liou. A robust and accurate approach to computing compressible multiphase flow: Stratified flow model and AUSM+-up scheme. Journal of Computational Physics, 225(1): 840-873, 2007.

[6] H. Chen and S. Liang. Flow visualization of shock/water column interactions. Shock Waves, 17(5): 309-321, 2008. 
[7] J. Cheng and C.-W. Shu. Positivity-preserving Lagrangian scheme for multi-material compressible flow. Journal of Computational Physics, 257:143-168, 2014.

[8] V. Coralic and T. Colonius. Finite-volume WENO scheme for viscous compressible multicomponent flows. Journal of computational physics, 274:95-121, 2014.

[9] X. Deng. New high-order hybrid cell-edge and cell-node weighted compact nonlinear schemes. In 20th AIAA Computational Fluid Dynamics Conference, page 3857, 2011.

[10] X. Deng and H. Zhang. Developing high-order weighted compact nonlinear schemes. Journal of Computational Physics, 165(1):22-44, 2000.

[11] B. Einfeldt, C.-D. Munz, P. L. Roe, and B. Sjögreen. On Godunov-type methods near low densities. Journal of Computational Physics, 92(2):273-295, 1991.

[12] L. Fu, X. Y. Hu, and N. A. Adams. A family of high-order targeted ENO schemes for compressiblefluid simulations. Journal of Computational Physics, 305:333-359, 2016.

[13] S. Gottlieb, C.-W. Shu, and E. Tadmor. Strong stability-preserving high-order time discretization methods. SIAM review, 43(1):89-112, 2001.

[14] S. Gottlieb, D. I. Ketcheson, and C.-W. Shu. High order strong stability preserving time discretizations. Journal of Scientific Computing, 38(3):251-289, 2009.

[15] J. Gressier, P. Villedieu, and J.-M. Moschetta. Positivity of flux vector splitting schemes. Journal of Computational Physics, 155(1):199-220, 1999.

[16] F. H. Harlow and A. A. Amsden. Fluid dynamics. Monograph LA-4700, Los Alamos National Lab., NM (United States), 1971.

[17] J. A. Housman, C. C. Kiris, and M. M. Hafez. Time-derivative preconditioning methods for multicomponent flows - part i: Riemann problems. Journal of applied mechanics, 76(2), 2009.

[18] J. A. Housman, C. C. Kiris, and M. M. Hafez. Time-derivative preconditioning methods for multicomponent flows - part ii: Two-dimensional applications. Journal of applied mechanics, 76(3), 2009.

[19] X. Hu, Q. Wang, and N. Adams. An adaptive central-upwind weighted essentially non-oscillatory scheme. Journal of Computational Physics, 229(23):8952-8965, 2010.

[20] X. Y. Hu, N. A. Adams, and C.-W. Shu. Positivity-preserving method for high-order conservative schemes solving compressible Euler equations. Journal of Computational Physics, 242:169-180, 2013.

[21] S. S. Jain, A. Mani, and P. Moin. A conservative diffuse-interface method for compressible two-phase flows. Journal of Computational Physics, page 109606, 2020.

[22] G.-S. Jiang and C.-W. Shu. Efficient implementation of weighted eno schemes. Journal of computational physics, 126(1):202-228, 1996.

[23] E. Johnsen and T. Colonius. Implementation of WENO schemes in compressible multicomponent flow problems. Journal of Computational Physics, 219(2):715-732, 2006.

[24] E. Johnsen, J. Larsson, A. V. Bhagatwala, W. H. Cabot, P. Moin, B. J. Olson, P. S. Rawat, S. K. Shankar, B. Sjögreen, H. C. Yee, X. Zhong, and S. K. Lele. Assessment of high-resolution methods for numerical simulations of compressible turbulence with shock waves. Journal of Computational Physics, 229(4):1213-1237, 2010.

[25] S. Kawai, S. K. Shankar, and S. K. Lele. Assessment of localized artificial diffusivity scheme for large-eddy simulation of compressible turbulent flows. Journal of Computational Physics, 229(5): $1739-1762,2010$

[26] C. C. Kiris, J. A. Housman, M. F. Barad, C. Brehm, E. Sozer, and S. Moini-Yekta. Computational framework for launch, ascent, and vehicle aerodynamics (LAVA). Aerospace Science and Technology, 55:189-219, 2016.

[27] T. Linde, P. Roe, T. Linde, and P. Roe. Robust Euler codes. In 13th Computational Fluid Dynamics Conference, page 2098, 1997.

[28] M.-S. Liou. A sequel to AUSM: AUSM+. Journal of computational Physics, 129(2):364-382, 1996

[29] T. Nonomura and K. Fujii. Effects of difference scheme type in high-order weighted compact nonlinear schemes. Journal of Computational Physics, 228(10):3533-3539, 2009.

[30] T. Nonomura and K. Fujii. Robust explicit formulation of weighted compact nonlinear scheme. Computers \& Fluids, 2013.

[31] T. Nonomura, N. Iizuka, and K. Fujii. Increasing order of accuracy of weighted compact nonlinear scheme. AIAA Paper, 893, 2007.

[32] G. Perigaud and R. Saurel. A compressible flow model with capillary effects. Journal of Computational Physics, 209(1):139-178, 2005.

[33] B. Perthame and C.-W. Shu. On positivity preserving finite volume schemes for euler equations. Numerische Mathematik, 73(1):119-130, 1996. 
[34] R. Saurel and R. Abgrall. A simple method for compressible multifluid flows. SIAM Journal on Scientific Computing, 21(3):1115-1145, 1999.

[35] R. Saurel and C. Pantano. Diffuse-interface capturing methods for compressible two-phase flows. Annual Review of Fluid Mechanics, 50:105-130, 2018.

[36] K. Sebastian and C.-W. Shu. Multidomain WENO finite difference method with interpolation at subdomain interfaces. Journal of Scientific Computing, 19(1-3):405-438, 2003.

[37] L. I. Sedov. Similarity and dimensional methods in mechanics. CRC press, 1993.

[38] S. Sembian, M. Liverts, N. Tillmark, and N. Apazidis. Plane shock wave interaction with a cylindrical water column. Physics of Fluids, 28(5):056102, 2016.

[39] H. Shen, C.-Y. Wen, M. Parsani, and C.-W. Shu. Maximum-principle-satisfying space-time conservation element and solution element scheme applied to compressible multifluids. Journal of Computational Physics, 330:668-692, 2017.

[40] C.-W. Shu. Total-variation-diminishing time discretizations. SIAM Journal on Scientific and Statistical Computing, 9(6):1073-1084, 1988.

[41] C.-W. Shu. High-order finite difference and finite volume weno schemes and discontinuous galerkin methods for cfd. International Journal of Computational Fluid Dynamics, 17(2):107-118, 2003.

[42] C.-W. Shu and S. Osher. Efficient implementation of essentially non-oscillatory shock-capturing schemes. Journal of computational physics, 77(2):439-471, 1988.

[43] K.-M. Shyue. A fluid-mixture type algorithm for compressible multicomponent flow with van der Waals equation of state. Journal of Computational Physics, 156(1):43-88, 1999.

[44] A. Subramaniam, M. L. Wong, and S. K. Lele. A high-order weighted compact high resolution scheme with boundary closures for compressible turbulent flows with shocks. Journal of Computational Physics, 397:108822, 2019.

[45] H. Takahira, T. Matsuno, and K. Shuto. Numerical investigations of shock-bubble interactions in mercury. Fluid Dynamics Research, 40(7-8):510, 2008.

[46] V. A. Titarev and E. F. Toro. Finite-volume WENO schemes for three-dimensional conservation laws. Journal of Computational Physics, 201(1):238-260, 2004.

[47] B. T. Vu, N. Bachchan, O. Peroomian, and V. Akdag. Multiphase modeling of water injection on flame deflector. In 21st AIAA computational fluid dynamics conference, page 2592, 2013.

[48] B. Wang, G. Xiang, and X. Y. Hu. An incremental-stencil WENO reconstruction for simulation of compressible two-phase flows. International Journal of Multiphase Flow, 104:20-31, 2018.

[49] M. L. Wong and S. K. Lele. High-order localized dissipation weighted compact nonlinear scheme for shock-and interface-capturing in compressible flows. Journal of Computational Physics, 339: 179-209, 2017.

[50] Z. Yan, H. Liu, M. Mao, H. Zhu, and X. Deng. New nonlinear weights for improving accuracy and resolution of weighted compact nonlinear scheme. Computers \&S Fluids, 127:226-240, 2016.

[51] S. Zhang, S. Jiang, and C.-W. Shu. Development of nonlinear weighted compact schemes with increasingly higher order accuracy. Journal of Computational Physics, 227(15):7294-7321, 2008.

[52] X. Zhang and C.-W. Shu. On maximum-principle-satisfying high order schemes for scalar conservation laws. Journal of Computational Physics, 229(9):3091-3120, 2010.

[53] X. Zhang and C.-W. Shu. On positivity-preserving high order discontinuous Galerkin schemes for compressible Euler equations on rectangular meshes. Journal of Computational Physics, 229(23): $8918-8934,2010$

[54] X. Zhang and C.-W. Shu. Positivity-preserving high order discontinuous Galerkin schemes for compressible Euler equations with source terms. Journal of Computational Physics, 230(4):12381248,2011

[55] X. Zhang and C.-W. Shu. Positivity-preserving high order finite difference WENO schemes for compressible Euler equations. Journal of Computational Physics, 231(5):2245-2258, 2012. 


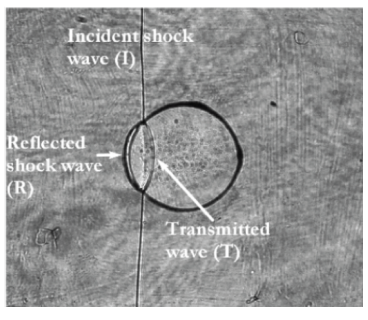

(a) Experiment

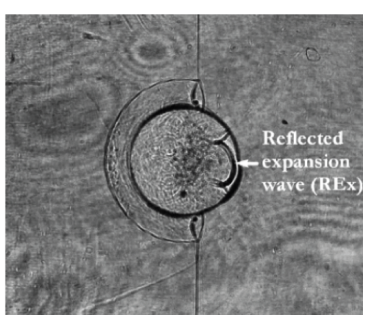

(c) Experiment

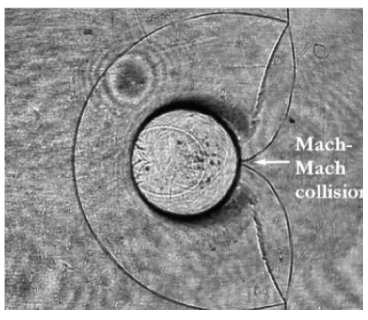

(e) Experiment

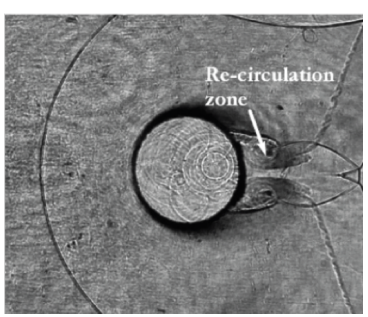

(g) Experiment

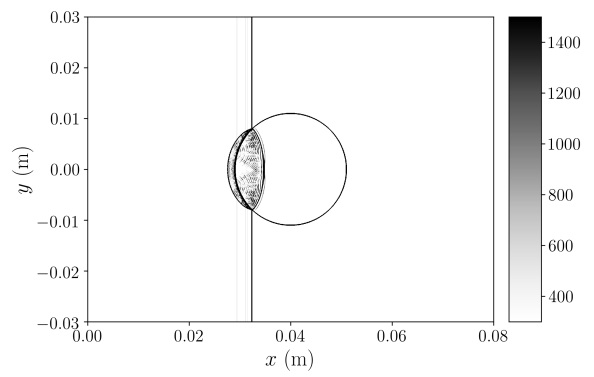

(b) $t=4 \mu \mathrm{s}$, PP-WCNS-IS

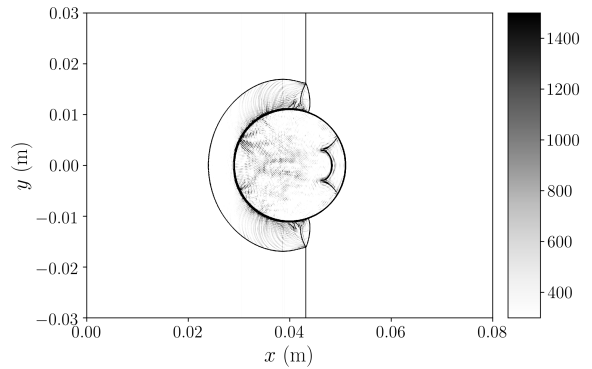

(d) $t=17 \mu \mathrm{s}$, PP-WCNS-IS

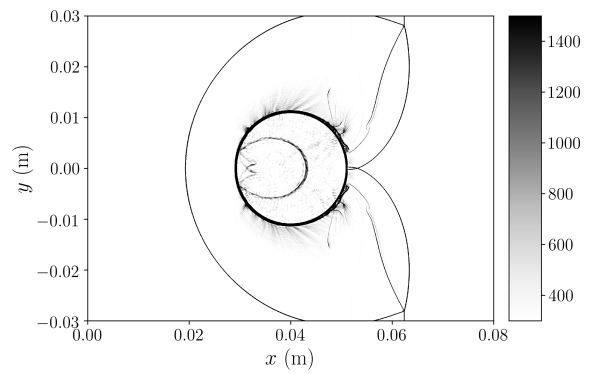

(f) $t=40 \mu \mathrm{s}$, PP-WCNS-IS

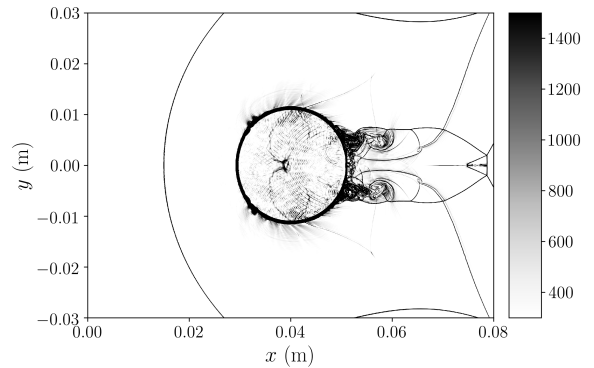

(h) $t=67 \mu \mathrm{s}$, PP-WCNS-IS

Figure 12: Comparision of 2D Mach 2.4 shock water cylinder interaction problem by Sembian et al. [38]. Left: shadowgraph images from [38]; right: density gradient from simulation with PP-WCNS-IS. 


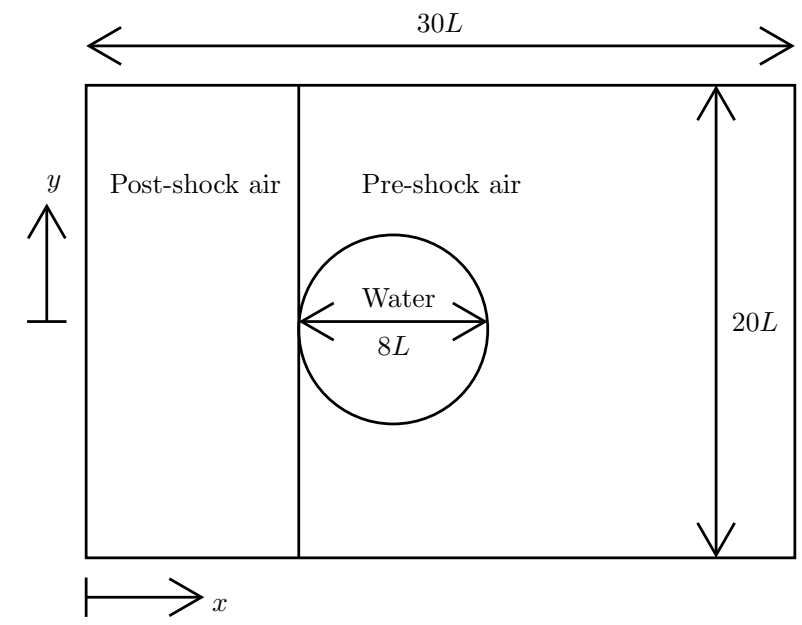

Figure 13: Schematic diagram of 2D Mach 10 shock water cylinder interaction problem. 


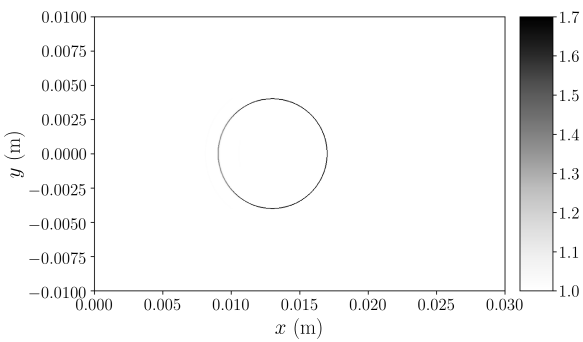

(a) $t=1 \mu \mathrm{s}, \mathrm{HLLC}$

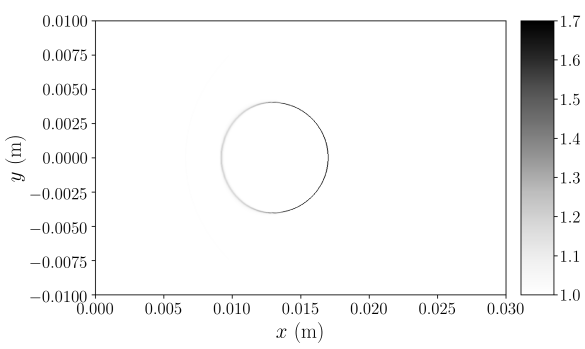

(c) $t=4 \mu \mathrm{s}$, HLLC

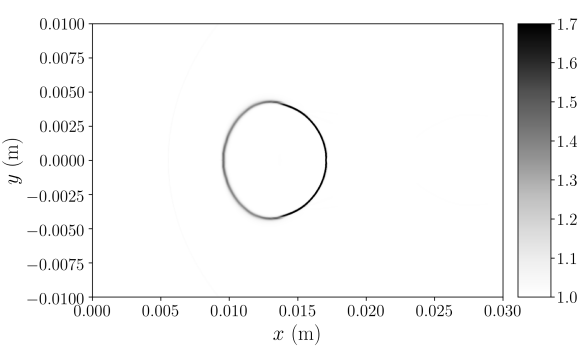

(e) $t=8 \mu \mathrm{s}$, HLLC

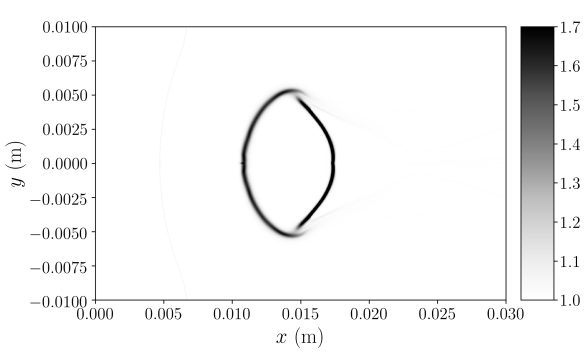

(g) $t=16 \mu \mathrm{s}$, HLLC

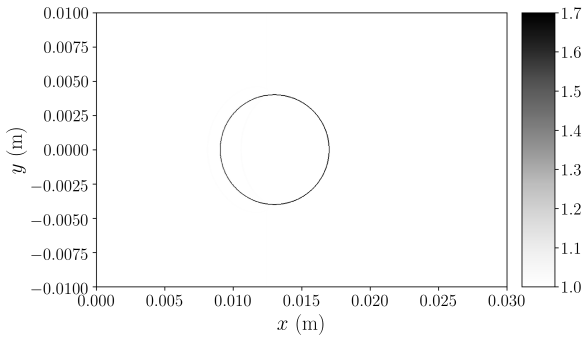

(b) $t=1 \mu \mathrm{s}$, PP-WCNS-IS

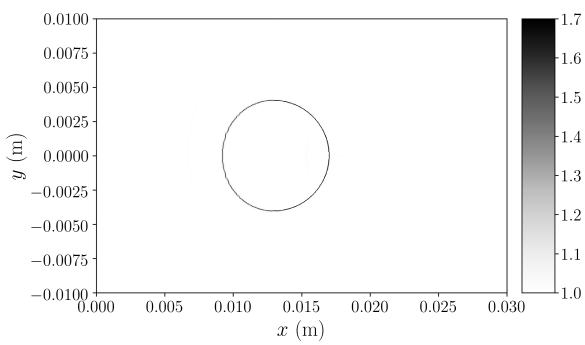

(d) $t=4 \mu \mathrm{s}$, PP-WCNS-IS

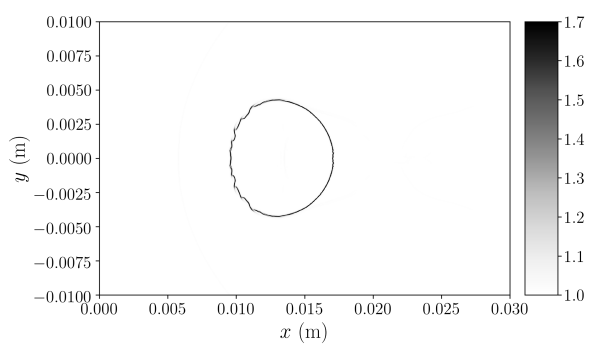

(f) $t=8 \mu \mathrm{s}$, PP-WCNS-IS

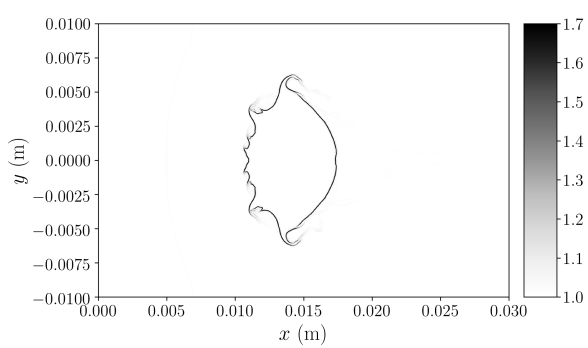

(h) $t=16 \mu \mathrm{s}$, PP-WCNS-IS

Figure 14: Numerical schlieren $\left(\exp \left(|\nabla \rho| /|\nabla \rho|_{\max }\right)\right)$ of 2D Mach 10 shock water cylinder interaction problem. 


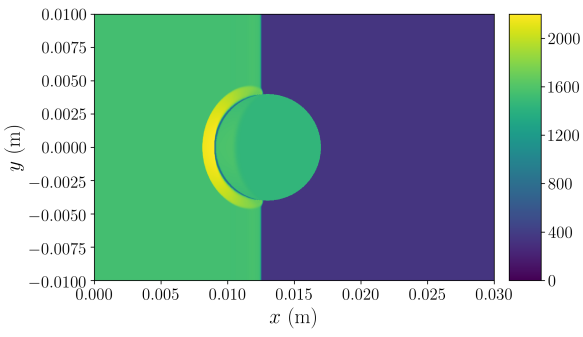

(a) $t=1 \mu \mathrm{s}$, HLLC

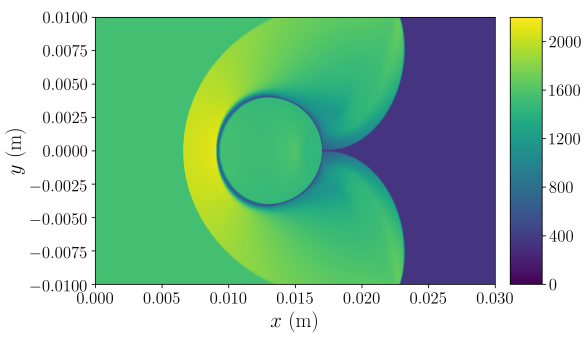

(c) $t=4 \mu \mathrm{s}$, HLLC

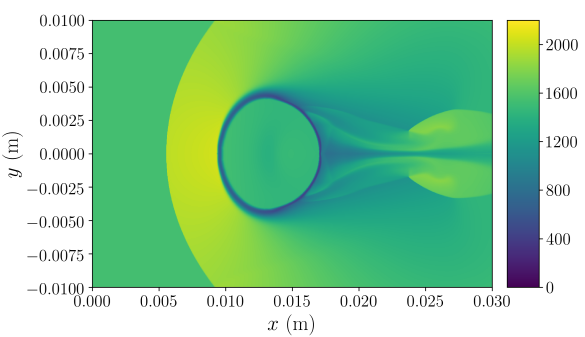

(e) $t=8 \mu \mathrm{s}$, HLLC

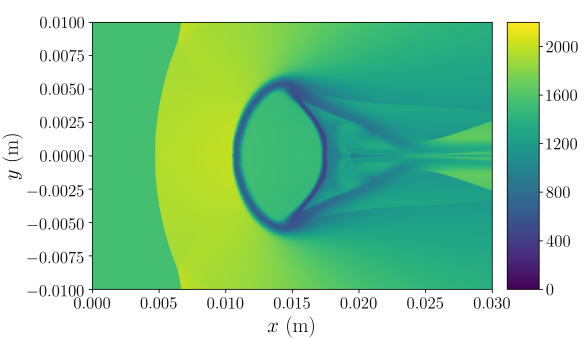

(g) $t=16 \mu \mathrm{s}$, HLLC

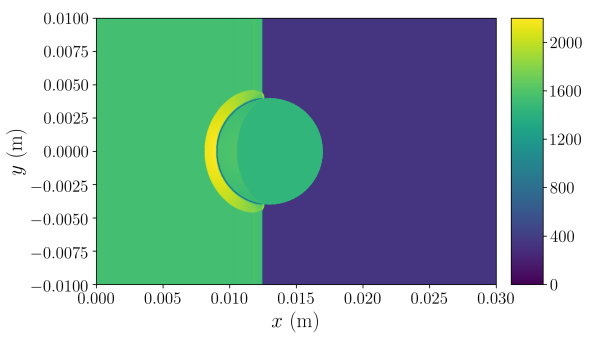

(b) $t=1 \mu \mathrm{s}$, PP-WCNS-IS

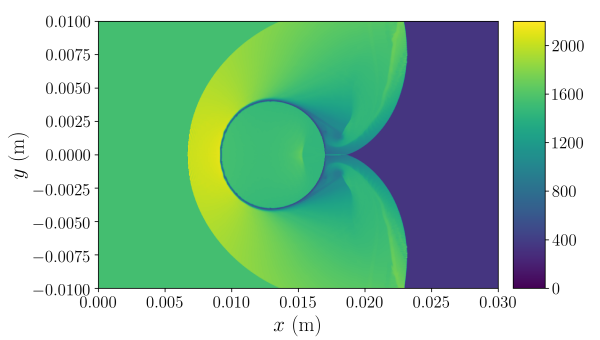

(d) $t=4 \mu \mathrm{s}$, PP-WCNS-IS

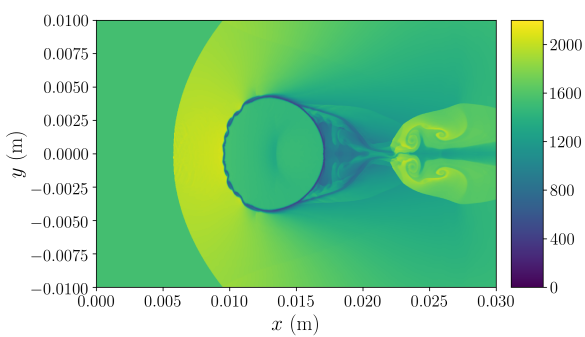

(f) $t=8 \mu \mathrm{s}$, PP-WCNS-IS

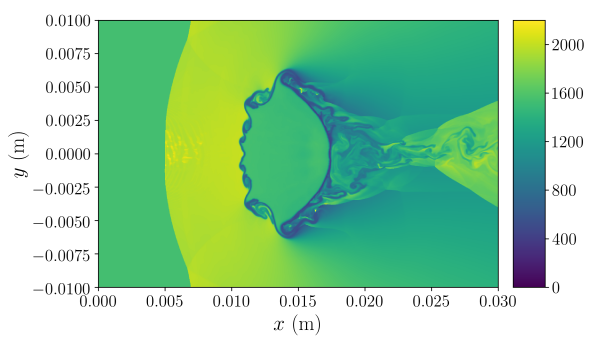

(h) $t=16 \mu \mathrm{s}$, PP-WCNS-IS

Figure 15: Speed of sound of 2D Mach 10 shock water cylinder interaction problem. 


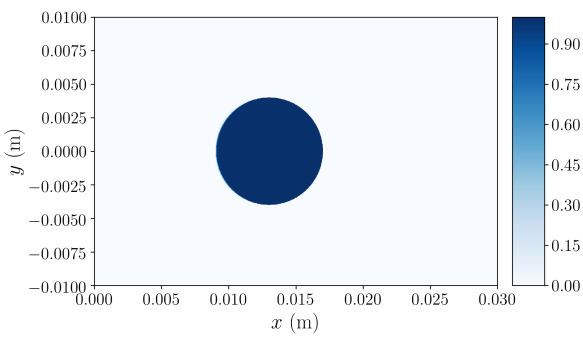

(a) $t=1 \mu \mathrm{s}$, HLLC

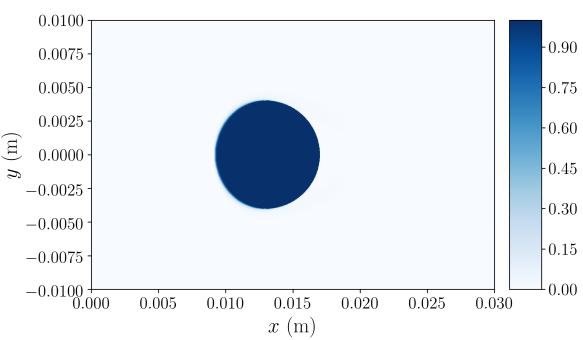

(c) $t=4 \mu \mathrm{s}$, HLLC

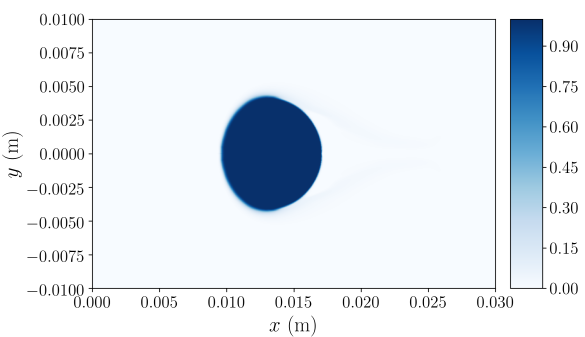

(e) $t=8 \mu \mathrm{s}$, HLLC

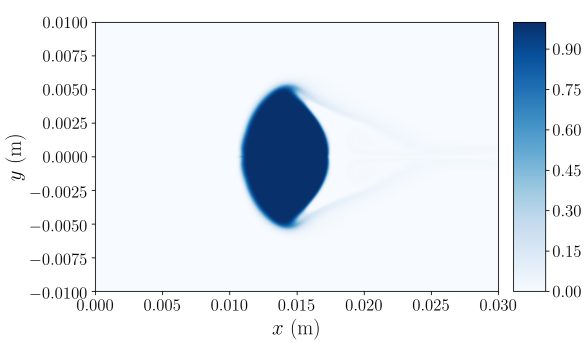

(g) $t=16 \mu \mathrm{s}$, HLLC

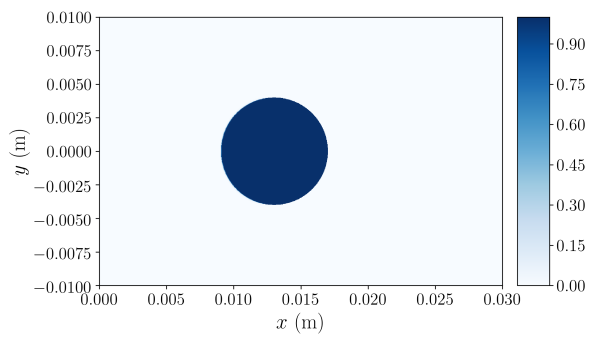

(b) $t=1 \mu \mathrm{s}$, PP-WCNS-IS

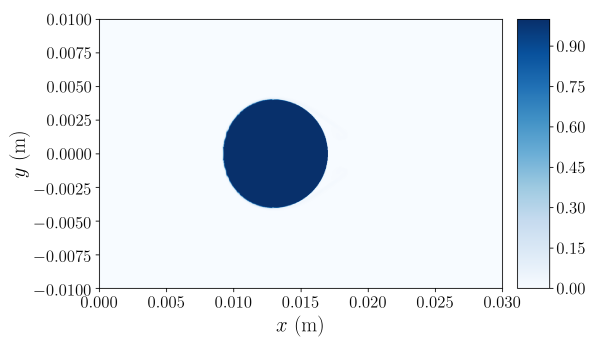

(d) $t=4 \mu \mathrm{s}$, PP-WCNS-IS

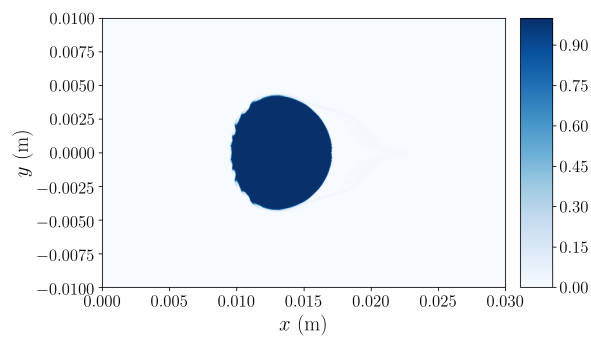

(f) $t=8 \mu \mathrm{s}$, PP-WCNS-IS

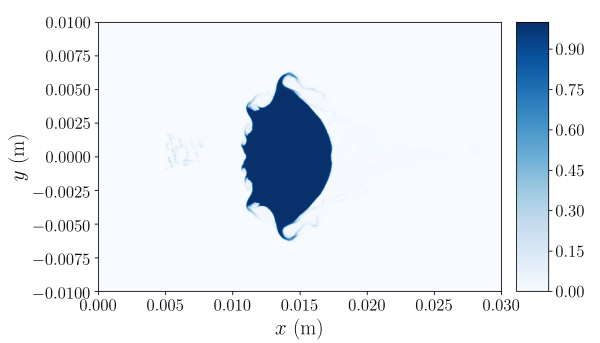

(h) $t=16 \mu \mathrm{s}$, PP-WCNS-IS

Figure 16: Volume fraction of water of 2D Mach 10 shock water cylinder interaction problem. 


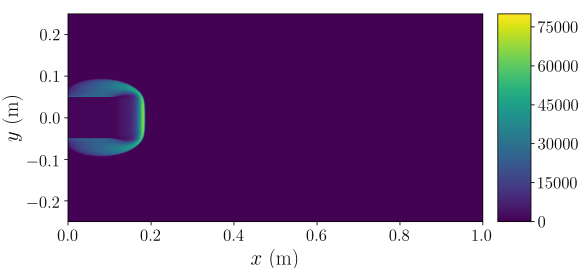

(a) $t=1 \mu \mathrm{s}$, HLLC

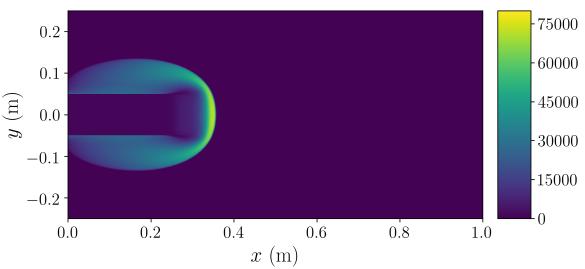

(c) $t=2 \mu \mathrm{s}$, HLLC

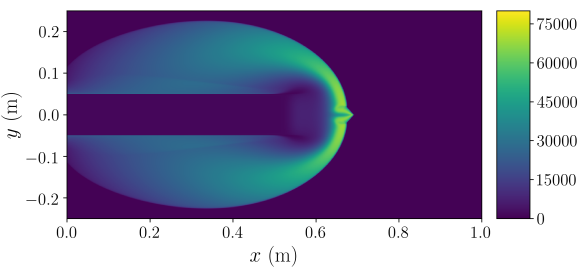

(e) $t=4 \mu \mathrm{s}$, HLLC

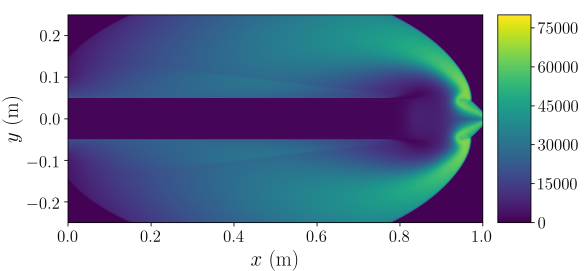

(g) $t=6 \mu \mathrm{s}$, HLLC

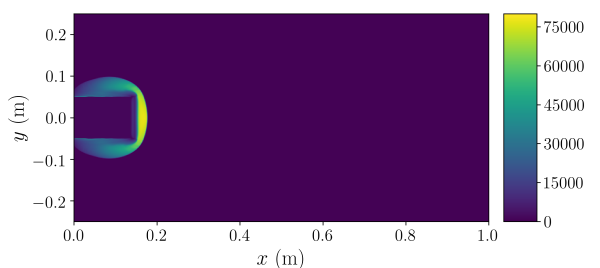

(b) $t=1 \mu \mathrm{s}$, PP-WCNS-IS

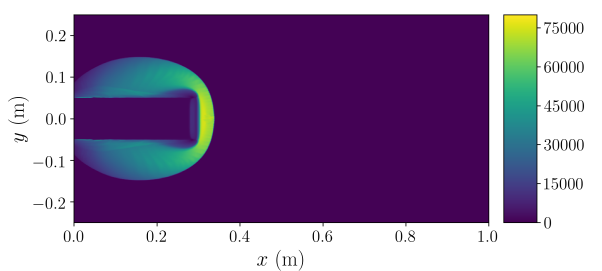

(d) $t=2 \mu \mathrm{s}$, PP-WCNS-IS

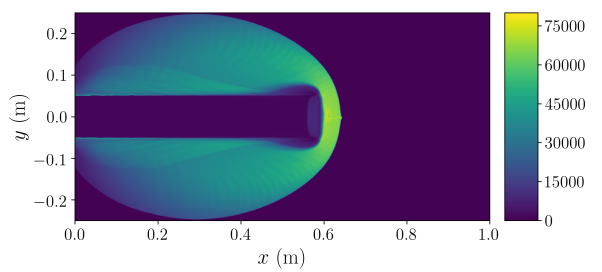

(f) $t=4 \mu \mathrm{s}$, PP-WCNS-IS

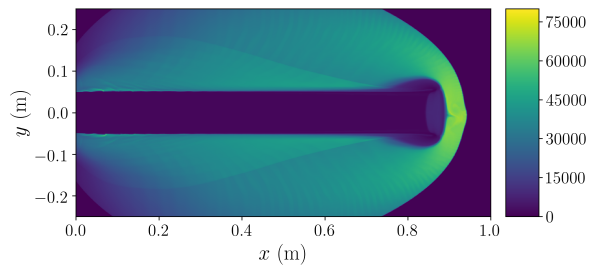

(h) $t=6 \mu \mathrm{s}$, PP-WCNS-IS

Figure 17: Speed of sound of 2D Mach 100 water jet problem. 


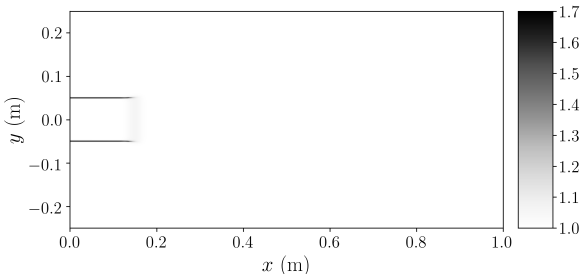

(a) $t=1 \mu \mathrm{s}$, HLLC

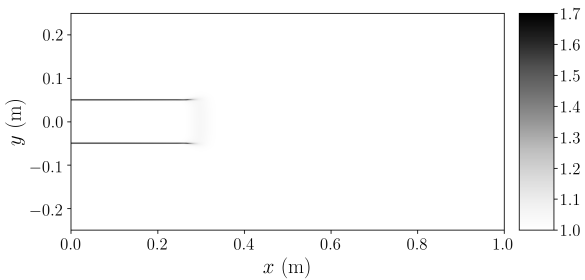

(c) $t=2 \mu \mathrm{s}, \mathrm{HLLC}$

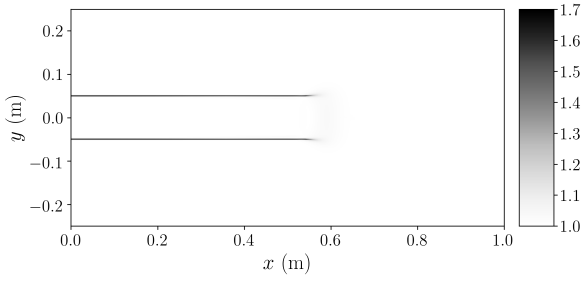

(e) $t=4 \mu \mathrm{s}$, HLLC

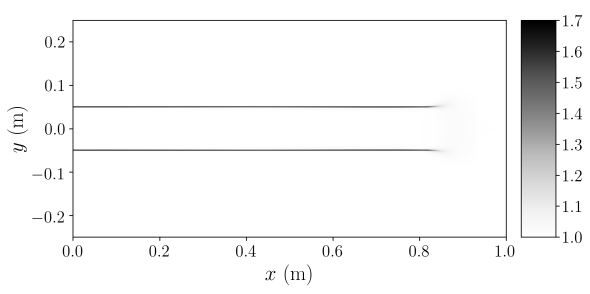

(g) $t=6 \mu \mathrm{s}$, HLLC

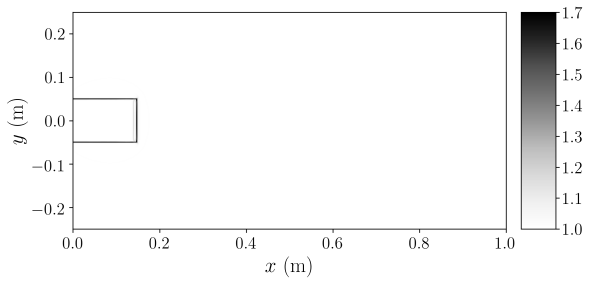

(b) $t=1 \mu \mathrm{s}$, PP-WCNS-IS

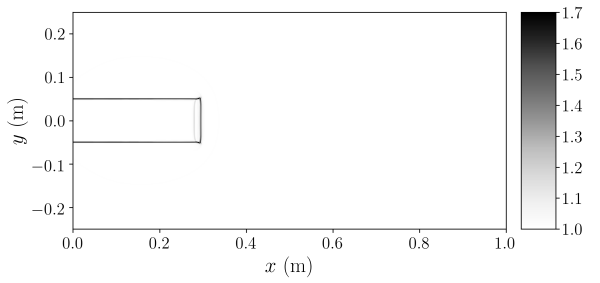

(d) $t=2 \mu \mathrm{s}$, PP-WCNS-IS

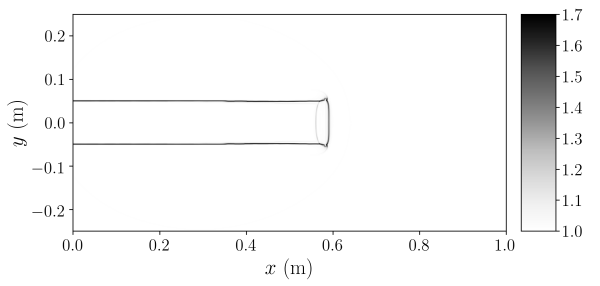

(f) $t=4 \mu \mathrm{s}$, PP-WCNS-IS

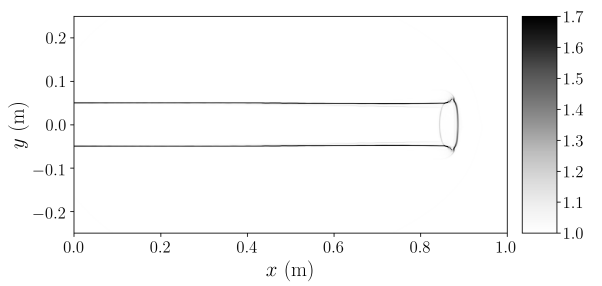

(h) $t=6 \mu \mathrm{s}$, PP-WCNS-IS

Figure 18: Numerical schlieren $\left(\exp \left(|\nabla \rho| /|\nabla \rho|_{\max }\right)\right)$ of 2D Mach 100 water jet problem. 


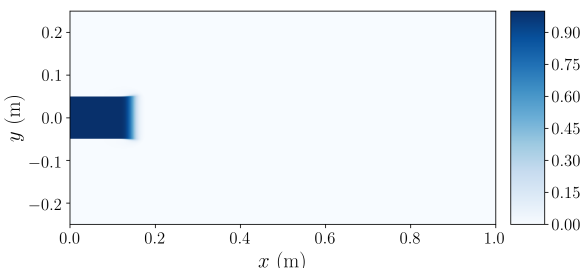

(a) $t=1 \mu \mathrm{s}, \mathrm{HLLC}$

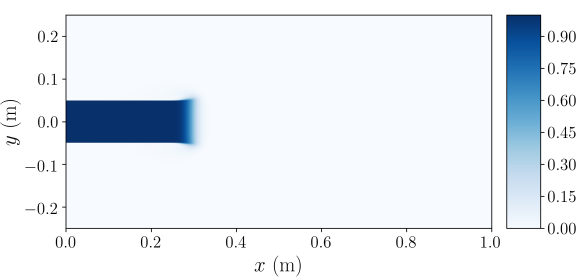

(c) $t=2 \mu \mathrm{s}$, HLLC

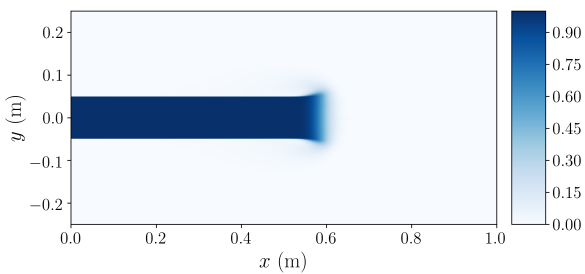

(e) $t=4 \mu \mathrm{s}$, HLLC

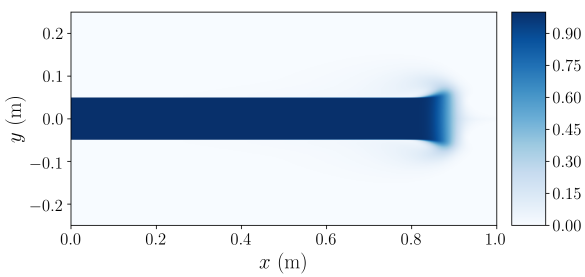

(g) $t=6 \mu \mathrm{s}$, HLLC

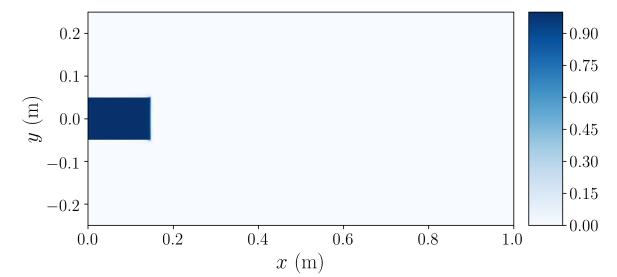

(b) $t=1 \mu \mathrm{s}$, PP-WCNS-IS

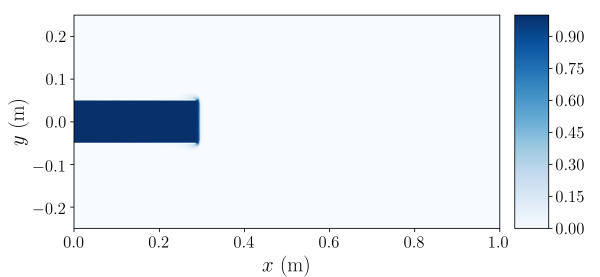

(d) $t=2 \mu \mathrm{s}$, PP-WCNS-IS

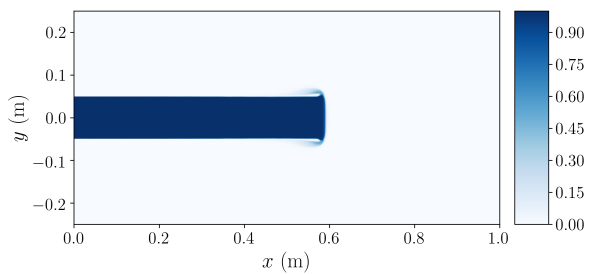

(f) $t=4 \mu \mathrm{s}$, PP-WCNS-IS

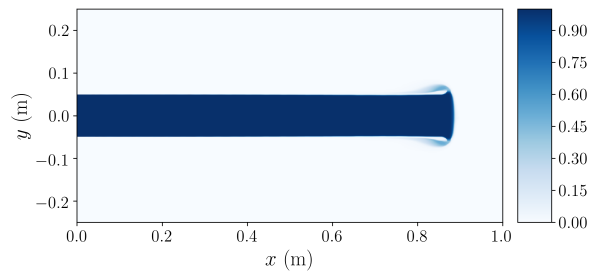

(h) $t=6 \mu \mathrm{s}$, PP-WCNS-IS

Figure 19: Volume fraction of water of 2D Mach 100 water jet problem. 